INSTITUT NATIONAL DE RECHERCHE EN INFORMATIQUE ET EN AUTOMATIQUE

\title{
On the Expressiveness of Pure Safe Ambients
}

Pascal Zimmer

$\mathbf{N}^{\circ} 4350$

Janvier 2002

THÈME 1 



\title{
On the Expressiveness of Pure Safe Ambients
}

\author{
Pascal Zimmer \\ Thème 1 - Réseaux et systèmes \\ Projet Mimosa \\ Rapport de recherche $\mathrm{n}^{\circ} 4350$ - Janvier $2002-54$ pages
}

\begin{abstract}
We consider the Pure Safe Ambient Calculus, which is Levi and Sangiorgi's Safe Ambient Calculus (a variant of Cardelli and Gordon's Mobile Ambient Calculus) restricted to its mobility primitives, and we focus on its expressive power. Since it has no form of communication or substitution, we show how these notions can be simulated by mobility and modifications in the hierarchical structure of ambients. As a main result, we use these techniques to design an encoding of the synchronous $\pi$-calculus into pure ambients, and we study its correctness, thus showing that pure ambients are as expressive as the $\pi$-calculus. In order to simplify the proof and give an intuitive understanding of the encoding, we design an intermediate language: the $\pi$-Calculus with Explicit Substitutions and Channels, which is an extension of the $\pi$-calculus in which communication and substitution are broken into simpler steps, and we show that is has the same expressive power as the $\pi$-calculus.
\end{abstract}

Key-words: Mobile Ambients, Safe Ambients, $\pi$-calculus, concurrent and mobile systems, expressiveness 


\section{Etude de l'Expressivité des Ambients Mobiles Purs}

Résumé : Nous étudions le Calcul des Ambients Purs, qui correspond au Calcul des Ambients de Cardelli et Gordon restreint aux seules primitives de mouvement, et nous nous intéressons plus particulièrement à sa puissance expressive. Etant donné qu'il ne possède aucune forme de communication ni de substitution, nous montrons comment ces notions peuvent être simulées par la mobilité et des modifications de la structure hiérarchique des ambients. Comme résultat principal, nous utilisons ces techniques pour construire un encodage du $\pi$-calcul synchrone dans les ambients purs et nous étudions sa correction, prouvant par conséquent que les ambients purs sont aussi expressifs que le $\pi$-calcul. Afin de simplifier la preuve et de faciliter la compréhension intuitive de l'encodage, nous introduisons un langage intermédiaire: le $\pi$-Calcul avec Substitutions et Canaux Explicites, qui est une extension du $\pi$-calcul dans laquelle la communication et la substitution sont décomposées en étapes plus élémentaires, et nous montrons qu'il possède aussi la même puissance expressive que le $\pi$-calcul.

Mots-clés : Ambients Mobiles, Safe Ambients, $\pi$-calcul, systèmes concurrents et mobiles, expressivité 


\section{Introduction}

The ambient calculus [Cardelli and Gordon, 1997, Cardelli and Gordon, 1998] was designed to model within a single framework both mobile computing, that is, computation in mobile devices like a laptop, and mobile computation, that is, mobile code moving between different devices, like applets or agents. It also shows how the notions of administrative domains, firewalls, authorizations... can be formalized in a calculus. (For more discussion about the problems raised by mobility and computation over wide-area networks, see [Cardelli, 1999a, Cardelli, 1999b].) Informally, an ambient is a bounded place where computation happens. Ambients can be nested so as to form a hierarchy. Each of them has a name (not necessarily distinct from other ambient names), which is used to control access. An ambient can be moved as a whole with all the computations and subambients it contains: it can enter another ambient or exit it. It can also be opened so that its contents get visible at the current level, and communication between two processes can occur within an ambient (like in the $\pi$-calculus).

As a variant, the safe ambients were first presented in [Levi and Sangiorgi, 2000]. They differ from the classical mobile ambients by the addition of coactions. In the ambient calculus, a movement is initiated only by the moving ambient and the target ambient has no control over it. On the contrary, in safe ambients both participants must agree by using matching action and coaction. In our attempts, it appeared that protocols were much simpler to implement in safe ambients than in classical ambients. For example, when designing a communication mechanism based on requests answered by replicated servers (both being ambients), it is difficult to prevent a server from answering twice the same request. In safe ambients, the uniqueness of answer is easier to achieve if there is only one coaction in each request.

The purpose of this paper is to study the expressive power of the subcalculus obtained by removing all communication primitives, the pure safe ambient calculus. This subcalculus has no abstraction at all: it has neither output nor input prefix, no variable binding, no communication rule, and it cannot perform any substitution of variables globally in a process. Consequently, the only "tools" allowed are the hierarchical structure of ambients, their movements and openings. The main motivation for this study is to understand what makes the ambient calculus so expressive and which constructs are really important from a purely theoretical point of view. A similar question arose in previous work in the setting of the $\pi$-calculus [Palamidessi, 1997]. After all, the pure ambient calculus is to the classical ambient calculus what CCS is to the $\pi$-calculus: the former has no operator of abstraction and no instantiation of variables, while the latter does.

We could not show that pure ambients were as expressive as classical mobile ambients, but we managed to encode the finite sum-free synchronous $\pi$-calculus [Milner, 1991] in pure (safe) ambients. Such a result is also interesting: we know that the $\pi$-calculus is very expressive, and we show that pure ambients are at least as expressive. We give such an encoding in this paper and prove its correctness. The main problem we had to face was the simulation of substitution (which is precisely what is missing in pure ambients): the communication rule of the $\pi$-calculus binds a variable $x$ to an output value $m$ and performs

$\mathrm{RR} \mathrm{n}^{\circ} 4350$ 
this substitution in the continuation process in one single step. With pure ambients, we need to adopt another mechanism: every future reference to $x$ has to be replaced dynamically by a reference to $m$. For this purpose, we create an ambient $x$ acting as a "forwarder". Furthermore, we introduce explicit channels in the form of unique ambients for each channel name, so that matching input and output primitives can meet somewhere.

It has been shown in [Cardelli and Gordon, 1998] that mobile ambients without communication primitives were expressive enough to simulate Turing machines. However, Turing machines are a good model for sequential programming but are not well adapted in a concurrency framework. What we want is a "reasonable" encoding having at least the property of compositionality (i.e. such that $\langle\langle P \mid Q\rangle\rangle=\langle\langle P\rangle\rangle \mid\langle\langle Q\rangle\rangle$ ), which would not be the case if we use an encoding via Turing machines (CCS is also Turing-complete, yet the $\pi$-calculus is much more powerful).

In order to show an operational correspondence between the $\pi$-calculus and our encoding, we had to design an intermediate calculus to simplify the proof, the $\pi$-Calculus with Explicit Substitutions and Channels ( $\pi_{e s c}$-calculus in short). It is an extension of the $\pi$-calculus, with new primitives for variables and explicit channels, breaking up the communication and substitution mechanisms of the $\pi$-calculus into simpler steps. This calculus appears to be an interesting byproduct and not only a technical tool, because:

- it allows a better intuitive description of the mechanism underlying the encoding in pure ambients;

- and it has the same expressive power as the $\pi$-calculus. More precisely, we give translations from $\pi$ to $\pi_{e s c}$ and vice versa, prove their correctness, and show a soundness result.

\subsection{Related Work}

Some encodings of the $\pi$-calculus into ambients have already been proposed in the literature [Cardelli and Gordon, 1998, Levi and Sangiorgi, 2000], but all of them encoded the communications and substitutions of the $\pi$-calculus into communications and substitutions of the ambient calculus, whereas our encoding cannot use these mechanisms. Moreover, all of them encoded only the asynchronous $\pi$-calculus $\left(\pi_{a}\right)$ and could not be easily extended so as to encode its synchronous version. Finally, except for the encoding of Levi and Sangiorgi [Levi and Sangiorgi, 2000], no operational correspondence result was ever completely proved for any of them.

For some restrictions of the $\pi$-calculus, substitution can be simulated in a different way from our approach. The local $\pi(L \pi)$ [Merro and Sangiorgi, 1998] is an asynchronous $\pi$ calculus (without matching) with an additional constraint on the input construct $n(x) . P$ : the name $x$ may not occur free in $P$ in input position. In this calculus, the following is a valid algebraic law:

$$
P\left\{{ }^{b} / c\right\}=(\nu c)(P \mid c \triangleleft b)
$$


where $c$ may not be free in $P$ in input position, $b \neq c$ and $c \triangleleft b \triangleq ! c(x) \cdot \bar{b}\langle x\rangle$ is a link forwarding every message for $c$ to $b$. Note that this law is false in the full $\pi_{a}$-calculus, hence also in the $\pi$-calculus, so we could not use this approach in our case (i.e. in the full synchronous $\pi$-calculus).

In the same way, an equator was first defined in [Honda and Yoshida, 1995] by:

$$
\mathcal{E}(b, c) \triangleq b \triangleleft c \mid c \triangleleft b
$$

and it was shown in [Merro, 1999] that

$$
P\left\{{ }^{b} / c\right\} \cong_{\pi_{a}}(\nu c)(\mathcal{E}(b, c) \mid P)
$$

( $\cong_{\pi_{a}}$ being barbed congruence in the $\pi_{a}$-calculus). However, this equality is false in the full synchronous $\pi$-calculus because the use of forwarders breaks the sequentiality imposed by output prefixing, so we could not use this approach either.

Some variants of the $\pi$-calculus with explicit substitutions were also proposed. In the $\pi \xi$-calculus [Ferrari et al., 1996], processes are prefixed by a global environment $\xi$ which contains the name associations carried on in past communications. The main rule is:

$$
\frac{P \stackrel{\omega}{\longrightarrow} P^{\prime}}{\xi:: P \stackrel{\delta\left(\xi, \xi^{\prime}, \omega\right)}{\longmapsto} \xi^{\prime}:: P^{\prime}} \quad \text { with } \quad \xi^{\prime} \in \eta(\xi, \omega)
$$

where the functions $\delta$ and $\eta$ are defined according to the desired semantics (late, early, open), such that the environment $\xi$ is extended with the name associations activated by the transition $P \stackrel{\omega}{\longrightarrow} P^{\prime}$. The main difference of this approach with our $\pi_{e s c}$-calculus is that there is only one global environment outside the process, instead of multiple variables directly included in the syntax and taking advantage of name restriction. Moreover, in the $\pi \xi$-calculus, substitutions are performed outside the term (in $\left.\delta\left(\xi, \xi^{\prime}, \omega\right)\right)$ and are not included in the reductions.

Another variant is the calculus of explicit substitutions $\pi \sigma$ from [Hirschkoff, 1999], in which a rewrite system is used to perform name substitutions inside terms. Since processes are written in De Bruijn notation, this calculus looks very different from the $\pi_{e s c}$-calculus. Furthermore, it performs substitutions in the whole output term (the rule is $(\bar{a} b)[s] \rightarrow$ $\overline{a[s]} b[s]$ ), so that the transitive closure of substitutions is automatically computed, whereas in the $\pi_{e s c}$-calculus, an arbitrary long chain of variables can be created. Moreover, the operational semantics of both $\pi \xi$ and $\pi \sigma$ are defined via a labelled transition system, whereas our calculus uses CHAM-style rules, and none of them introduces explicit channels in its syntax.

A final remark is that all dialects and variants of the $\pi$-calculus which have been studied so far have a construct for abstraction (usually embodied in the input prefix), hence computation involves some form of substitution. For us, the challenge consisted precisely in the fact that we do not have any such operator in pure ambients.

$\mathrm{RR} \mathrm{n}^{\circ} 4350$ 


\subsection{Outline}

In Section 2, we give the necessary background on the $\pi$-calculus and safe ambients. We also introduce a special kind of substitution. In Section 3, we present the $\pi_{e s c}$-calculus and some associated tools. Section 4 defines encodings between the $\pi$-calculus and the $\pi_{e s c}$-calculus, states the main relations between them and gives an overview of the proofs. The second part of the encoding, from the $\pi_{e s c}$-calculus into pure ambients, is given in Section 5, together with an operational correspondence result. Finally, Section 6 gathers the results into a main theorem and gives the final encoding for the $\pi$-calculus. Proofs of the results stated in this paper are given in Appendix A.

\section{Background}

\subsection{The $\pi$-Calculus}

We start by reviewing the syntax of the monadic synchronous $\pi$-calculus we will use throughout the paper.

\subsubsection{Syntax}

We distinguish between names of channels and names of variables. Let Name be a denumerably infinite set of names of channels (ranged over by $n, m, p, \ldots$ ), and $V a r$ a denumerably infinite set of names of variables (ranged over by $x, y, \ldots$ ). We need to treat those two sets as distinct, because their behaviour will be different in the encoding into ambients. The syntax of the $\pi$-calculus is then defined as follows:

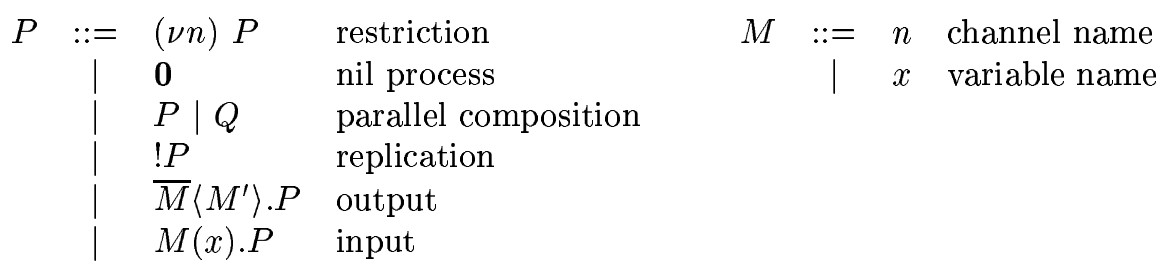

In $(\nu n) P$ and $M(x) . P$, the names $n$ and $x$ respectively are bound in $P$. We can always change this name using $\alpha$-conversion, and we consider the resulting process equal to the first one. If a name is not bound, it is called free. The set of free channel names of $P$ is denoted by $f n(P)$, and the set of free variable names by $f v(P)$.

\subsubsection{Reduction Rules}

Below is the operational semantics of our $\pi$-calculus, given in the form of an one-step reduction relation, written $\longrightarrow$. The main rule is $(\pi$ Red Comm) in which an input prefix and an output prefix on a same channel $n$ are consumed, whereas the variable $x$ is replaced by the 
value $m$ (the construction $Q\{m / x\}$ is defined as the result of replacing each free occurrence of $x$ in $Q$ by $m$ ). We write $\longrightarrow^{*}$ for zero or more reductions in the $\pi$-calculus, and $\longrightarrow^{+}$ for one or more reductions (same conventions apply also to the other calculi that will be presented in the rest of the paper).

$$
\begin{aligned}
& \overline{\bar{n}\langle m\rangle \cdot P|n(x) \cdot Q \longrightarrow P| Q\{m / x}\} \quad(\pi \text { Red Comm }) \\
& \frac{P \longrightarrow P^{\prime}}{P\left|Q \longrightarrow P^{\prime}\right| Q}(\pi \text { Red Par }) \\
& \frac{P \longrightarrow P^{\prime}}{(\nu n) P \longrightarrow(\nu n) P^{\prime}} \quad(\pi \text { Red Res }) \\
& \frac{P \equiv P^{\prime} \longrightarrow Q^{\prime} \equiv Q}{P \longrightarrow Q}(\pi \text { Red Struct })
\end{aligned}
$$

The last rule of this one-step reduction makes use of a structural congruence rewriting relation $\equiv$. Its definition is standard, with rules to commute processes in parallel, to change the scope of a restriction operator, unfold a replicated process, ... Its rules are given below.

$$
\begin{array}{cl}
P \equiv P & (\pi \text { Struct Refl }) \\
P \equiv Q \Rightarrow Q \equiv P & (\pi \text { Struct Symm }) \\
P \equiv Q \equiv R \Rightarrow P \equiv R & (\pi \text { Struct Trans }) \\
P \equiv Q \Rightarrow(\nu n) P \equiv(\nu n) Q & (\pi \text { Struct Res }) \\
P \equiv Q \Rightarrow P|R \equiv Q| R & (\pi \text { Struct Par }) \\
P \equiv Q \Rightarrow ! P \equiv ! Q & (\pi \text { Struct Repl }) \\
P \equiv Q \Rightarrow \bar{M}\left\langle M^{\prime}\right\rangle \cdot P \equiv \bar{M}\left\langle M^{\prime}\right\rangle \cdot Q & (\pi \text { Struct Output) } \\
P \equiv Q \Rightarrow M(x) \cdot P \equiv M(x) \cdot Q & (\pi \text { Struct Input }) \\
P \mid \mathbf{0} \equiv P & (\pi \text { Struct Par Zero }) \\
& \\
P|Q \equiv Q| P & (\pi \text { Struct Par Comm }) \\
P|(Q \mid R) \equiv(P \mid Q)| R & (\pi \text { Struct Par Assoc }) \\
(\nu n)(P \mid Q) \equiv P \mid(\nu n) Q \text { if } n \notin f n(P) & (\pi \text { Struct Res Par }) \\
(\nu n)(\nu m) P \equiv(\nu m)(\nu n) P & (\pi \text { Struct Res Res }) \\
! P \equiv P \mid ! P & (\pi \text { Struct Repl Par }) \\
! \mathbf{0} \equiv \mathbf{0} & (\pi \text { Struct Repl Zero })
\end{array}
$$$$
! P \equiv P \mid ! P
$$

\subsection{Pure Ambients}

We present here the subcalculus of the Safe Ambient Calculus we will use. It corresponds to the original Safe Ambients from [Levi and Sangiorgi, 2000] with the communication primitives removed. This restriction allows us to simplify the syntax (the original one needed a 
type system to reject some ill-formed terms). The complete syntax is defined as follows.

\begin{tabular}{l|ll}
$P \quad:=$ & $(\nu n) P$ & restriction \\
& $\mathbf{0}$ & nil process \\
& $P \mid Q$ & parallel composition \\
& $! P$ & replication \\
& $n[P]$ & ambient \\
Cap.P & capability
\end{tabular}

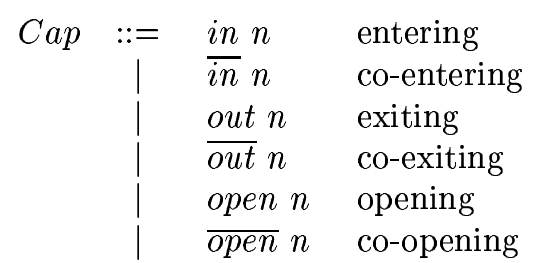

The basic constructs of process calculi are present: restriction of names, nil process, parallel operator and replication. They behave as in the $\pi$-calculus. An ambient is written $n[P]$ where $n$ is the name of the ambient and $P$ is the process running inside it. Actions are called capabilities and are written Cap.P. There are three possible capabilities: one to enter an ambient ( in $n$ ), one to exit an ambient ( out $n$ ) and one to open an ambient (open $n$ ), each of them having a corresponding cocapability (namely $\overline{\text { in }} n, \overline{\text { out }} n$ and $\overline{\text { open }} n$ ). In order for a movement to take place, two corresponding capability and cocapability (that is, with the same name) must be present at the right place, as shown by the following reduction rules:

$$
\begin{aligned}
& n[\text { in } m . P \mid Q] \mid m[\overline{i n} m \cdot R \mid S] \hookrightarrow m[n[P \mid Q]|R| S] \quad \text { (SA Red In) } \\
& m[n[\text { out } m . P \mid Q]|\overline{\text { out }} m . R| S] \hookrightarrow n[P \mid Q] \mid m[R \mid S] \quad \text { (SA Red Out) } \\
& \text { open } n . P \mid n[\text { open } n . Q] \hookrightarrow P \mid Q \quad \text { (SA Red Open) }
\end{aligned}
$$

The operational semantics is completed by four other rules, so that reduction can occur under restriction, in parallel processes, inside ambients, or after a structural congruence rewriting (which is very similar to the structural congruence for the $\pi$-calculus).

$$
\begin{aligned}
& \frac{P \hookrightarrow Q}{(\nu n) P \hookrightarrow(\nu n) Q} \text { (SA Red Res) } \quad \frac{P \hookrightarrow Q}{P|R \hookrightarrow Q| R} \text { (SA Red Par) } \\
& \frac{P \hookrightarrow Q}{n[P] \hookrightarrow n[Q]} \text { (SA Red Amb) } \frac{P \equiv P^{\prime} \quad P^{\prime} \hookrightarrow Q^{\prime} \quad Q^{\prime} \equiv Q}{P \hookrightarrow Q} \text { (SA Red Struct) }
\end{aligned}
$$

The main difference with the Safe Ambients of [Levi and Sangiorgi, 2000] is the lack of communication primitives, namely the asynchronous output $\langle M\rangle$ and the input binder $(x) . P$. Another difference is the use of replication in place of recursion. Furthermore, cocapabilities are not present in the Ambient Calculus of [Cardelli and Gordon, 1998].

\subsection{Substitutions}

In this Section, we introduce a special kind of substitution, having a tree structure. This is needed because both $\pi_{e s c}$-calculus and the encoding into ambients implicitely use such a mathematical structure and not a substitution of general shape.

Intuitively, to every variable, it associates either another variable in the domain of the substitution, or a channel name. And there is an additional condition: by following the "chain" of successive images, we always end on a channel name. 
More formally, in the rest of the paper, every occurrence of "substitution" refers to the following definition:

Definition 1 A substitution is a partial function $\sigma: \operatorname{Var} \rightarrow \operatorname{Var} \cup$ Name such that:

- $\forall x \in \operatorname{dom}(\sigma), x \sigma \in N a m e \cup \operatorname{dom}(\sigma)$ (i.e. $\operatorname{im}(\sigma) \subseteq \operatorname{Name} \cup \operatorname{dom}(\sigma)$ )

- $\forall x \in \operatorname{dom}(\sigma)$, there is $k>0$ such that $x \sigma^{k} \in$ Name (i.e. there are no cycles) ( $\sigma^{k}$ being the composition of $\sigma, k$ times)

Let us define the graph of a substitution: its set of vertices is dom $(\sigma) \cup$ Name and its edges are $(x, x \sigma)$ for $x \in \operatorname{dom}(\sigma)$. With the above definition, one can easily show that the graph of a substitution has a forest structure (a set of trees), with roots in Name and all other nodes in $\operatorname{dom}(\sigma) \subseteq V a r$. Consequently, we can define $\sigma^{*}: \operatorname{dom}(\sigma) \rightarrow$ Name, the transitive closure of $\sigma$, associating to each variable the name at the root of the corresponding tree $\left(\sigma^{*}=\sigma^{p}\right.$ where $p=\max \left\{k / x \sigma^{k} \in\right.$ Name, $\left.\left.x \in \operatorname{dom}(\sigma)\right\}\right)$.

If $x \notin \operatorname{dom}(\sigma)$ and $M \in N$ ame $\cup \operatorname{dom}(\sigma)$, we define $\sigma^{\prime}=\left\{{ }^{M} / x\right\} \uplus \sigma$ by $x \sigma^{\prime}=M$ and $y \sigma^{\prime}=y \sigma$ for $y \neq x$. The resulting substitution $\sigma^{\prime}$ is still a substitution in the sense of Definition 1.

The empty substitution is written $\varnothing$, and we also define $f n(\sigma) \triangleq i m(\sigma) \cap$ Name. Moreover, we extend the domain of substitutions so that we can apply them to processes.

\section{The Intermediate Calculus $\left(\pi_{e s c}\right)$}

In this Section, we introduce our $\pi$-Calculus with Explicit Substitutions and Channels.

\subsection{Syntax}

Syntactically, the $\pi_{e s c}$-calculus is an extension of the $\pi$-calculus, with additional constructs to handle substitutions and channels.

First, the construction $(\nu x: M) P($ with $x \neq M$ ) represents a new variable $x$ whose contents is $M$. The name $x$ is bound in $P$ (as $n$ is bound in $(\nu n) P$ ). Intuitively, any free occurrence of the name $x$ in $P$ refers to this variable and can be replaced by $M$ without changing the behaviour of the process $P$.

Prefixes in the $\pi$-calculus have the form $M(x) . P$ and $\bar{M}\left\langle M^{\prime}\right\rangle . P$; we call the bodies of the prefixes, namely $(x) . P$ and $\left\langle M^{\prime}\right\rangle . P$, abstraction and concretion, respectively.

The construction $[n: S]$ represents an explicit channel of name $n$, whose contents are a set $S$ of abstractions and concretions performed on that channel. More precisely, $S$ is not exactly a set but a parallel composition of abstractions and concretions (we use parallel composition for convenience in proofs). $S$ can be either $\varepsilon$ (the empty channel), a parallel composition $S \mid S^{\prime}$, a concretion $\langle M\rangle . P$ for an output, or an abstraction $(x) . P$ for an input (they correspond respectively to the processes $\bar{n}\langle M\rangle . P$ and $n(x) . P)$. Intuitively, when a process performs an output or input on $n$, the request is put inside the channel with that name (if there is one). 
The complete syntax of $\pi_{e s c}$ is the following one:

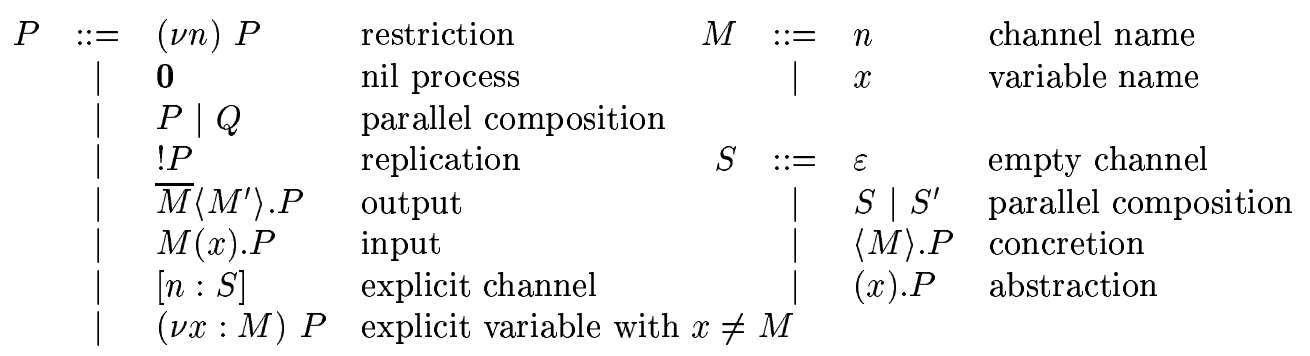

\subsection{Reduction Rules}

We give now an operational semantics for $\pi_{e s c}$. Reduction rules are of the form $\sigma: P \longmapsto P^{\prime}$, where $P$ and $P^{\prime}$ are processes, and $\sigma$ is a substitution that acts as an environment containing the values of free variables in $P$. As a side condition, we restrict the application of the rules to processes $P$ and substitutions $\sigma$ such that $f v(P) \subseteq \operatorname{dom}(\sigma)$, so that we can find the value of every free variable appearing in $P$.

The first two rules allow us to replace an output or input prefix on a variable $x$ by the same prefix on the value $M$ of $x$. If $M$ is another variable, we can then apply the same rule again (since in this case $M \in \operatorname{dom}(\sigma)$ by definition of a substitution). We continue like this until $M$ is a channel name. Note also that we do not perform substitutions on $M^{\prime}$ in the rule $\left(\pi_{e s c}\right.$ Red Subst Out).

$$
\begin{gathered}
\frac{x \sigma=M}{\sigma: \bar{x}\left\langle M^{\prime}\right\rangle . P \longmapsto \bar{M}\left\langle M^{\prime}\right\rangle . P}\left(\pi_{e s c} \text { Red Subst Out }\right) \\
\frac{x \sigma=M}{\sigma: x(y) . P \longmapsto M(y) . P}\left(\pi_{e s c} \text { Red Subst In }\right)
\end{gathered}
$$

The next two rules were already outlined above: if a channel $n$ and a prefixed process on $n$ meet in a parallel composition, the request is put inside the channel (we then omit the name $n$ since all abstractions and concretions in $[n: S]$ refer implicitly to $n$ ).

$$
\begin{gathered}
\overline{\sigma:[n: S] \mid \bar{n}\langle M\rangle \cdot P \longmapsto[n: S \mid\langle M\rangle \cdot P]} \\
\overline{\sigma:[n: S] \mid n(x) . P \longmapsto[n: S \mid(x) \cdot P]}\left(\pi_{e s c} \text { Red Output }\right)
\end{gathered}
$$

When a concretion $\langle M\rangle . P$ and an abstraction $(x) \cdot Q$ are present in the same channel, communication can effectively occur. The two continuations $P$ and $Q$ are then placed outside the channel, except that a new variable $x$ with contents $M$ is created in front of $Q$. This is the purpose of the following rule, which corresponds to ( $\pi$ Red Comm) (the side condition $x \neq M$ can always be satisfied by $\alpha$-conversion on $x$ ). 


$$
\frac{x \neq M}{\sigma:[n: S \mid(\langle M\rangle \cdot P \mid(x) \cdot Q)] \longmapsto[n: S] \mid(P \mid(\nu x: M) Q)} \quad\left(\pi_{e s c}\right. \text { Red Comm) }
$$

The next rule allows a reduction to occur under a variable restriction $(\nu x: M)$. The only side-effect is that the binding $\left\{{ }^{M} / x\right\}$ must be added to the environment $\sigma$ (the side condition $x \notin \operatorname{dom}(\sigma)$ can always be satisfied by $\alpha$-conversion on $x$, and the condition $M \in N a m e \cup \operatorname{dom}(\sigma)$ is automatically satisfied because $f v((\nu x: M) P) \subseteq \operatorname{dom}(\sigma)$, which is an instance of the implicit side condition).

$$
\frac{x \notin \operatorname{dom}(\sigma) \quad\left\{{ }^{M} / x\right\} \uplus \sigma: P \longmapsto P^{\prime}}{\sigma:(\nu x: M) P \longmapsto(\nu x: M) P^{\prime}}\left(\pi_{e s c} \operatorname{Red} \text { Var }\right)
$$

Finally, the last three rules complete the calculus: reduction can occur under the scope restriction of a channel name, in a parallel composition or by means of a structural congruence rewriting.

$$
\begin{aligned}
& \frac{\sigma: P \longmapsto P^{\prime}}{\sigma: P\left|Q \longmapsto P^{\prime}\right| Q} \quad\left(\pi_{e s c} \text { Red Par }\right) \\
& \frac{\sigma: P \longmapsto P^{\prime}}{\sigma:(\nu n) P \longmapsto(\nu n) P^{\prime}} \quad\left(\pi_{e s c} \text { Red Res }\right) \\
& \frac{P \equiv P^{\prime} \quad \sigma: P^{\prime} \longmapsto Q^{\prime} \quad Q^{\prime} \equiv Q}{\sigma: P \longmapsto Q}\left(\pi_{e s c} \text { Red Struct }\right)
\end{aligned}
$$

\subsection{Structural Congruence}

The congruence $\equiv$ is the same as in the $\pi$-calculus, with additional rules for the new constructs and their interaction with the old ones (in particular the scope of $(\nu x: M)$ can be stretched or commuted with $(\nu n)$ provided that there are no name captures). Its complete list is given below:

$$
\begin{aligned}
& P \equiv P \quad(\text { same for } S) \\
& P \equiv Q \Rightarrow Q \equiv P \quad(\text { same for } S) \\
& P \equiv Q \equiv R \Rightarrow P \equiv R \quad(\text { same for } S) \\
& P \equiv Q \Rightarrow(\nu n) P \equiv(\nu n) Q \\
& P \equiv Q \Rightarrow P|R \equiv Q| R \\
& P \equiv Q \Rightarrow ! P \equiv ! Q \\
& P \equiv Q \Rightarrow \bar{M}\left\langle M^{\prime}\right\rangle \cdot P \equiv \bar{M}\left\langle M^{\prime}\right\rangle \cdot Q \\
& P \equiv Q \Rightarrow M(x) \cdot P \equiv M(x) \cdot Q \\
& S \equiv S^{\prime} \Rightarrow[n: S] \equiv\left[n: S^{\prime}\right] \\
& P \equiv Q \Rightarrow(\nu x: M) P \equiv(\nu x: M) Q \\
& S^{\prime} \equiv S^{\prime \prime} \Rightarrow S\left|S^{\prime} \equiv S\right| S^{\prime \prime} \\
& P \equiv Q \Rightarrow\langle M\rangle \cdot P \equiv\langle M\rangle \cdot Q \\
& P \equiv Q \Rightarrow(x) \cdot P \equiv(x) \cdot Q \\
& P \mid \mathbf{0} \equiv P
\end{aligned}
$$$$
\begin{aligned}
& \left(\pi_{e s c} \text { Struct Refl }\right) \\
& \left(\pi_{e s c} \text { Struct Symm }\right) \\
& \left(\pi_{e s c} \text { Struct Trans }\right) \\
& \left(\pi_{e s c} \text { Struct Res }\right) \\
& \left(\pi_{e s c} \text { Struct Par }\right) \\
& \left(\pi_{e s c} \text { Struct Repl }\right) \\
& \left(\pi_{e s c} \text { Struct Output }\right) \\
& \left(\pi_{e s c} \text { Struct Input }\right) \\
& \left(\pi_{e s c} \text { Struct Channel }\right) \\
& \left(\pi_{e s c} \text { Struct Var }\right) \\
& \left(\pi_{e s c} \text { Struct Abs }\right) \\
& \left(\pi_{e s c} \text { Struct Out Abs }\right) \\
& \left(\pi_{e s c} \text { Struct In Abs }\right) \\
& \left(\pi_{e s c} \text { Struct Par Zero }\right)
\end{aligned}
$$ 


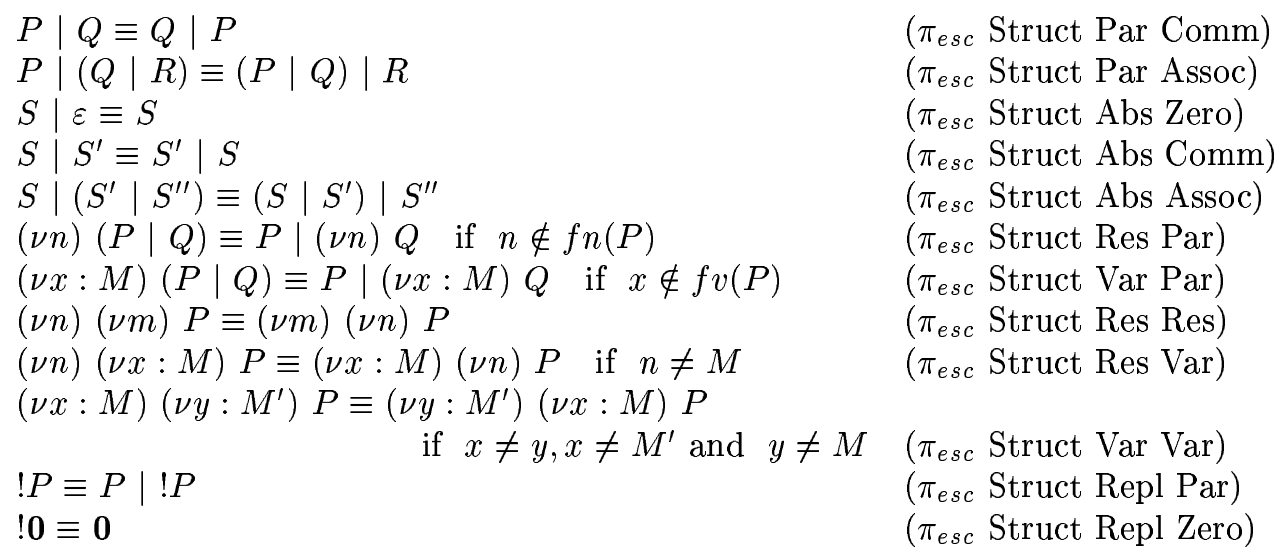

\subsection{Channel Presentation}

To follow our intuition (i.e. the modelling of explicit channels), we need to cut down the set of allowed processes in the $\pi_{e s c}$-calculus to ensure that channels are correctly positioned and unique. Consider for instance the process $\bar{n}\langle m\rangle \cdot[p: S]$. The channel $p$ would be unreachable, and thus useless, until the output on $n$ has been performed. Consider also the following process:

$$
[n: S]\left|\left[n: S^{\prime}\right]\right| \bar{n}\langle m\rangle . P \mid n(x) . Q
$$

Since there are two channels, the two prefixed processes could go into different channels, for instance resulting in

$$
[n: S \mid\langle m\rangle \cdot P] \mid\left[n: S^{\prime} \mid(x) \cdot Q\right]
$$

and communication would never occur between $P$ and $Q$.

For this reason, we need to be able to detect a channel. We define a presentation predicate $P \Downarrow_{1} n$, which means intuitively that at least a channel $[n: S]$ is present in $P$ and is not hidden by scope restriction. The formal definition of this predicate is easy: the only axiom is $[n: S] \Downarrow_{1} n$ and all other rules perform only inductive calls (except for $(\nu m) P \Downarrow_{1} n$ which checks $m \neq n$ ).

In the same way, we can define another predicate, $P \Downarrow_{2} n$, meaning that there are at least two different channels of name $n$ in $P$. For instance, $P \mid Q \Downarrow_{2} n$ holds if both $P \Downarrow_{1} n$ and $Q \Downarrow_{1} n$ hold at the same time.

Here is the formal definition of $P \Downarrow_{i} n$ where $i=1,2$ :

$$
\begin{array}{lr}
\frac{P \Downarrow_{i} n}{(\nu m) P \Downarrow_{i} n}\left(\pi_{e s c} \text { Pres Res }\right) & \frac{P \Downarrow_{i} n}{P \mid Q \Downarrow_{i} n}\left(\pi_{e s c} \text { Pres ParL }\right) \\
\frac{Q \Downarrow_{i} n}{P \mid Q \Downarrow_{i} n}\left(\pi_{e s c} \text { Pres ParR }\right) & \frac{P \Downarrow_{1} n \quad Q \Downarrow_{1} n}{P \mid Q \Downarrow_{2} n}\left(\pi_{e s c} \text { Pres } \operatorname{Par}_{2}\right)
\end{array}
$$




$$
\begin{aligned}
& \frac{P \Downarrow_{i} n}{! P \Downarrow_{i} n}\left(\pi_{e s c} \text { Pres Repl }\right) \quad \frac{P \Downarrow_{1} n}{! P \Downarrow_{2} n}\left(\pi_{e s c} \text { Pres Repl }_{2}\right) \\
& \frac{P \Downarrow_{i} n}{\bar{M}\left\langle M^{\prime}\right\rangle . P \Downarrow_{i} n}\left(\pi_{e s c} \text { Pres Output }\right) \quad \frac{P \Downarrow_{i} n}{M(x) . P \Downarrow_{i} n}\left(\pi_{e s c} \text { Pres Input }\right) \\
& \overline{[n: S] \Downarrow_{1} n}\left(\pi_{e s c} \text { Pres Channel }_{1}\right) \quad \frac{S \Downarrow_{1} n}{[n: S] \Downarrow_{2} n}\left(\pi_{e s c} \text { Pres Channel }{ }_{2}\right) \\
& \frac{S \Downarrow_{i} m}{[n: S] \Downarrow_{i} m}\left(\pi_{e s c} \text { Pres Channel }\right) \quad \frac{P \Downarrow_{i} n}{(\nu x: M) P \Downarrow_{i} n} \quad\left(\pi_{e s c} \text { Pres Var }\right) \\
& \frac{S \Downarrow_{1} n}{S \mid S^{\prime} \Downarrow_{1} n}\left(\pi_{e s c} \text { Pres AbsL }\right) \quad \frac{S^{\prime} \Downarrow_{1} n}{S \mid S^{\prime} \Downarrow_{1} n}\left(\pi_{e s c} \text { Pres AbsR }\right) \\
& \frac{S \Downarrow_{1} n \quad S^{\prime} \Downarrow_{1} n}{S \mid S^{\prime} \Downarrow_{2} n}\left(\pi_{e s c} \text { Pres } \mathrm{Abs}_{2}\right) \quad \frac{P \Downarrow_{i} n}{\langle M\rangle . P \Downarrow_{i} n}\left(\pi_{e s c} \text { Pres Out Abs }\right) \\
& \frac{P \Downarrow_{i} n}{(x) \cdot P \Downarrow_{i} n}\left(\pi_{e s c} \text { Pres In Abs }\right)
\end{aligned}
$$

Moreover, we write $\operatorname{pr}(P)$ for the set of channels presented by $P$.

Definition $2 \operatorname{pr}(P) \triangleq\left\{n \in N a m e / P \Downarrow_{1} n\right\}$

Proposition $3 \operatorname{pr}(P) \subseteq f n(P)$

Proof: See Section A.2.

\subsection{Validity}

Now that we have a way to detect the presence or absence of one or many channels, we can define exactly the set of valid processes by ensuring that all channels are correctly positioned and unique. This can be achieved by means of a small type system.

For this purpose, we define the predicate $\vdash P: O K$ inductively on $P$, by checking that channels do not appear after prefixes or replications, and that there is at most one channel after a name restriction.

$$
\begin{aligned}
& \frac{\vdash P: O K \quad P \Downarrow_{2} n}{\vdash(\nu n) P: O K}\left(\pi_{e s c} \text { OK Res }\right) \quad \overline{\vdash \mathbf{0}: O K}\left(\pi_{e s c} \text { OK Zero }\right) \\
& \frac{\vdash P: O K \quad \vdash Q: O K}{\vdash P \mid Q: O K}\left(\pi_{e s c} \text { OK Par }\right) \\
& \frac{\vdash P: O K \quad \forall n \in N \text { ame } P \not \psi_{1} n}{\vdash ! P: O K}\left(\pi_{e s c} \text { OK Repl }\right) \\
& \frac{\vdash P: O K \quad \forall n \in N a m e P \Downarrow_{1} n}{\vdash \bar{M}\left\langle M^{\prime}\right\rangle . P: O K}\left(\pi_{e s c} \text { OK Output }\right)
\end{aligned}
$$

$\mathrm{RR} \mathrm{n}^{\circ} 4350$ 


$$
\begin{aligned}
& \frac{\vdash P: O K \quad \forall n \in \text { Name } P \not \Downarrow_{1} n}{\vdash M(x) \cdot P: O K}\left(\pi_{e s c} \text { OK Input }\right) \\
& \frac{\vdash S: O K}{\vdash[n: S]: O K}\left(\pi_{e s c} \text { OK Channel }\right) \quad \frac{\vdash P: O K}{\vdash(\nu x: M) P: O K}\left(\pi_{e s c} \text { OK Var }\right) \\
& \overline{\vdash \varepsilon: O K}\left(\pi_{e s c} \text { OK Eps }\right) \quad \frac{\vdash S: O K \quad \vdash S^{\prime}: O K}{\vdash S \mid S^{\prime}: O K}\left(\pi_{e s c} \text { OK Abs }\right) \\
& \frac{\vdash P: O K \quad \forall n \in N a m e P \Downarrow \Downarrow_{1} n}{\vdash\langle M\rangle . P: O K}\left(\pi_{e s c} \text { OK Out Abs }\right) \\
& \frac{\vdash P: O K \quad \forall n \in N \text { ame } P \Downarrow_{1} n}{\vdash(x) . P: O K}\left(\pi_{e s c} \text { OK In Abs }\right)
\end{aligned}
$$

The following lemma details the syntactic structure of a process presenting a channel $n$ (after type-checking). This corresponds to the desired intuition: if $P \Downarrow_{1} n$, a channel $[n: S]$ is present at the highest level, i.e. only under some name restrictions.

Lemma 4 If $P \Downarrow_{1} n$ and $\vdash P: O K$, then $P \equiv\left(\nu n_{1}\right) \ldots\left(\nu n_{k}\right)\left(\nu x_{1}: M_{1}\right) \ldots\left(\nu x_{k^{\prime}}\right.$ : $\left.M_{k^{\prime}}\right)\left([n: S] \mid P^{\prime}\right)$ with $n \neq n_{i}$.

Proof: By induction on the structure of $P$.

Corollary 5 If $P \Downarrow_{1} n_{i}$ and $\vdash P: O K$, then $P \equiv\left(\nu m_{1}\right) \ldots\left(\nu m_{k}\right)\left(\nu x_{1}: M_{1}\right) \ldots\left(\nu x_{k^{\prime}}\right.$ : $\left.M_{k^{\prime}}\right)\left(\left[n_{1}: S_{1}\right]|\ldots|\left[n_{p}: S_{p}\right] \mid P^{\prime}\right)$ with $n_{i} \neq m_{j}$.

Proof: We give the inductive step by considering a process $P$ such that $P \Downarrow_{1} n_{1}$ and $P \Downarrow_{1} n_{2}$. By Lemma $4, P \equiv\left(\nu m_{1}\right) \ldots\left(\nu m_{k}\right)\left(\nu x_{1}: M_{1}\right) \ldots\left(\nu x_{k^{\prime}}: M_{k^{\prime}}\right)\left(\left[n_{1}: S_{1}\right] \mid P_{1}\right)$ with $m_{1} \neq n_{i}$. From $\vdash P: O K$, we get $\vdash S_{1}: O K$ and $\vdash P_{1}: O K$. Consequently, we cannot have $S_{1} \Downarrow_{1} n_{2}$. Necessarily, $P_{1} \Downarrow_{1} n_{2}$ since $P \Downarrow_{1} n_{2}$. Then apply Lemma 4 to $P_{1}$ and use scope extrusion to get the final result.

Finally, we say that a process $P$ is valid, and write $\vdash P:$ Valid, if $\vdash P: O K$ and $P \Downarrow_{2} n$ for all name $n \in$ Name.

$$
\frac{\vdash P: O K \quad \forall n \in \text { Name } P \Downarrow_{2} n}{\vdash P: \text { Valid }}\left(\pi_{e s c} \text { Valid }\right)
$$

From now on, we will focus mainly on valid processes only. The following proposition shows that this property is preserved by reduction.

Proposition 6 (Subject Reduction) If $\sigma: P \longmapsto Q$ and $\vdash P:$ Valid, then $\vdash Q:$ Valid.

Proof: See Section A.2. 


\subsection{Closure}

Now that we eliminated some extra channels, we will have to add a few ! Consider the process $\bar{n}\langle m\rangle . P \mid n(x) \cdot Q$. It cannot reduce because no explicit channel is present for $n$. If we put an empty channel $[n: \varepsilon]$ in parallel, communication takes place. We thus define the channel closure of a process by adding explicit empty channels when needed. Since the same problem can appear under a scope restriction (for instance, $(\nu n)(\bar{n}\langle m\rangle \cdot P \mid n(x) \cdot Q)$ cannot reduce), we need to take care of this case too.

Definition 7 We first take scope restrictions into account. $c l(P)$ is a homomorphism from $\pi_{\text {esc }}$-processes to $\pi_{\text {esc }}$-processes for all constructs, except for restriction:

$$
c l((\nu n) P) \triangleq \begin{cases}(\nu n)([n: \varepsilon] \mid \operatorname{cl}(P)) & \text { if } P \Downarrow_{1} n \\ (\nu n) c l(P) & \text { if } \quad P \Downarrow_{1} n\end{cases}
$$

Then, the channel closure of a process with regard to a substitution $\sigma$ consists in adding an empty channel for each free name in $P$ or $\sigma$ for which $P$ does not present a channel. Formally:

$$
\operatorname{cl}_{\sigma}(P) \triangleq\left[n_{1}: \varepsilon\right]|\ldots|\left[n_{k}: \varepsilon\right] \mid \operatorname{cl}(P)
$$

where $\left\{n_{1}, \ldots, n_{k}\right\}=(f n(P) \cup f n(\sigma)) \backslash \operatorname{pr}(P)$ (by Proposition 3, we know that $\operatorname{pr}(P) \subseteq$ $f n(P))$

We say that $P$ is channel-closed with regard to $\sigma$ if $c_{\sigma}(P) \equiv P$ (that is if $P$ has all channels to guarantee communication).

Note that, in Definition 7, if we take two different enumerations for $(f n(P) \cup f n(\sigma)) \backslash$ $\operatorname{pr}(P)$, the resulting processes may not be syntactically equal; they will only be structurally congruent. This is why all our results involving $c l_{\sigma}(P)$ will be up to $\equiv$.

\section{Relations between the $\pi$ and $\pi_{e s c}$-Calculi}

In this Section, we prove a few equivalence properties between the $\pi$-calculus and the $\pi_{e s c^{-}}$ calculus. The proofs mainly rely on our ability to translate a $\pi_{e s c}$-process back into a $\pi$-process.

\subsection{Back to the $\pi$-Calculus}

The translation from $\pi_{e s c}$ to $\pi$ is written $\llbracket P \rrbracket$ (with a parameter name $n$ for the content of a channel) and is defined inductively by the following rules:

$$
\begin{array}{ll}
\llbracket(\nu n) P \rrbracket=(\nu n) \llbracket P \rrbracket & \llbracket[n: S] \rrbracket=\llbracket S \rrbracket_{n} \\
\llbracket \mathbf{0} \rrbracket=\mathbf{0} & \llbracket(\nu x: M) P \rrbracket=\llbracket P \rrbracket\left\{\left.{ }^{M}\right|_{x}\right\} \\
\llbracket P|Q \rrbracket=\llbracket P \rrbracket| \llbracket Q \rrbracket & \llbracket \varepsilon \rrbracket_{n}=\mathbf{0} \\
\llbracket ! P \rrbracket=! \llbracket P \rrbracket & \llbracket\langle M\rangle \cdot P \rrbracket_{n}=\bar{n}\langle M\rangle \cdot \llbracket P \rrbracket \\
\llbracket \bar{M}\left\langle M^{\prime}\right\rangle \cdot P \rrbracket=\bar{M}\left\langle M^{\prime}\right\rangle \cdot \llbracket P \rrbracket & \llbracket(x) \cdot P \rrbracket_{n}=n(x) \cdot \llbracket P \rrbracket \\
\llbracket M(x) \cdot P \rrbracket=M(x) \cdot \llbracket P \rrbracket & \llbracket S\left|S^{\prime} \rrbracket_{n}=\llbracket S \rrbracket_{n}\right| \llbracket S^{\prime} \rrbracket_{n}
\end{array}
$$

$\mathrm{RR} \mathrm{n}^{\circ} 4350$ 
In fact, $\llbracket P \rrbracket$ is a homomorphism for all constructs, except for channels and variable restrictions. In the former case, we just have to add the name of the channel back in front of abstractions and concretions. In the latter case, we perform the substitution required by the variable restriction: that is, $\llbracket(\nu x: M) P \rrbracket$ is $\llbracket P \rrbracket$ in which we replace every free occurrence of $x$ by $M$.

\subsection{Operational Correspondence}

When should we say that a $\pi$-process and a $\pi_{e s c}$-process are "equivalent" ? Following our intuition, a $\pi_{e s c}$-process $P$ evolving in an environment $\sigma$ should be translated into the $\pi$ process $\llbracket P \rrbracket \sigma^{*}$. Here we need to take the bindings of $\sigma$ into account, because the free variables of $P$ coming from previous communications should be replaced by their value. We apply the transitive closure $\sigma^{*}$ in one step so that all free variables are converted into names of channels (in fact, $\llbracket P \rrbracket \sigma^{*}$ is equal to $\llbracket\left(\nu x_{1}: M_{1}\right) \ldots\left(\nu x_{k}: M_{k}\right) P \rrbracket$ if $\left.\sigma=\left\{{ }^{M_{k}} / x_{k}\right\} \uplus \ldots \uplus\left\{{ }^{M_{1}} / x_{1}\right\}\right)$.

The following technical proposition shows that every reduction step in the $\pi_{e s c}$-calculus corresponds to zero or one step in the $\pi$-calculus.

Proposition 8 If $\sigma: P \longmapsto Q$, then $\llbracket P \rrbracket \sigma^{*} \stackrel{\equiv}{\longrightarrow} \llbracket Q \rrbracket \sigma^{*}$, where $\stackrel{\equiv}{\longrightarrow}$ is either $\equiv$ or $\longrightarrow$.

Proof: By induction on the derivation of $\sigma: P \longmapsto Q$. See Section A.6.

The converse proposition is more complex. Additional hypotheses restrict the result to valid processes and appropriate environments only. The result states that every reduction step in the $\pi$-calculus can be simulated by one or more reduction steps in the $\pi_{e s c}$-calculus. Moreover, this simulation is not defined directly on $P$, but on its channel closure $c_{\sigma}(P)$ (for instance, the $\pi$-processes in Section 3.6 reduce in the $\pi$-calculus, but only their channel closures reduce in the $\pi_{e s c}$-calculus).

Proposition 9 If $\llbracket P \rrbracket \sigma^{*} \longrightarrow Q$, and $\vdash P:$ Valid, and $f v(P) \subseteq \operatorname{dom}(\sigma)$, then there is a process $P^{\prime}$ such that $\sigma: c l_{\sigma}(P) \longmapsto^{+} P^{\prime}$ and $\llbracket P^{\prime} \rrbracket \sigma^{*} \equiv Q$.

Proof: By induction of the derivation of $\llbracket P \rrbracket \sigma^{*} \longrightarrow Q$. See Section A.7.

This proposition is much more difficult to prove. We try to explain why and give a few hints.

- Channel closure does not mix well with an inductive proof. This comes from the fact that channel closure is not defined inductively on terms. Consequently, for almost every construct, we need a preliminary lemma that analyses this special case and relates the channel closure of the process with the channel closures of its sub-components. Sometimes, there is more than a single answer, depending on the context. See Section A.3.

- Empty channels do not mix well with structural congruence rewriting. For instance, if the first step of reduction is

$$
\llbracket[n: \varepsilon]\left|P \rrbracket \sigma^{*}=\mathbf{0}\right| \llbracket P \rrbracket \sigma^{*} \equiv \llbracket P \rrbracket \sigma^{*} \longrightarrow Q
$$


we cannot proceed directly by induction since the resulting process $P$ does not present channel $n$ anymore (structural congruence has "erased" it), hence the channel closures of $[n: \varepsilon] \mid P$ and $P$ are different. This example is simple, but in the general case, channel erasing can occur anywhere in a term. So we need a result to relate the channel closure of $P$ with $P^{\prime}$ when $\llbracket P \rrbracket \sigma^{*} \equiv P^{\prime}$ is the first step of reduction. See Section A.4.

- Channels do not mix well with parallel composition. This is the problem which needs the longest technical development. Suppose that $\llbracket P\left|P^{\prime} \rrbracket \sigma^{*} \longrightarrow Q\right| \llbracket P^{\prime} \rrbracket \sigma^{*}$ was derived from $\llbracket P \rrbracket \sigma^{*} \longrightarrow Q$ by ( $\pi$ Red Par). Suppose also that this reduction involves a communication on channel $n$, and that $P \Downarrow_{1} n$ and $P^{\prime} \Downarrow_{1} n$ (that is, the explicit channel $n$ is in the $P^{\prime}$ part). Therefore, by induction, we get a simulation on $c l_{\sigma}(P)=[n: \varepsilon] \mid P_{1}$ since $P \Downarrow_{1} n$. But now the corresponding reductions of $c l_{\sigma}\left(P \mid P^{\prime}\right)$ involving channel $n$ should use the explicit channel in $P^{\prime}$ and not the empty channel $[n: \varepsilon]$ we added in the channel closure! In the general case, we need a result showing that reductions involving empty channels from closure can be replaced by reductions where communications are reported on (possibly non-empty) channels from a process in parallel. See Section A.5.

These are technical propositions, but in the rest of this paper, we restrict ourselves to valid processes, without free variables and channel-closed with regard to $\varnothing$. Since we use those processes extensively throughout the paper, we will call them complete:

Definition $10 A \pi_{e s c}$-process $P$ is called complete if $P$ is channel-closed with regard to $\varnothing$, $\vdash P:$ Valid and $f v(P)=\emptyset$.

In this case, the operational correspondence is much simpler:

\section{Corollary 11}

- If $\varnothing: P \longmapsto Q$, then $\llbracket P \rrbracket \stackrel{\equiv}{\longrightarrow} \llbracket Q \rrbracket$.

- If $\llbracket P \rrbracket \longrightarrow Q$ for a complete process $P$, then there is a process $P^{\prime}$ such that $\varnothing$ : $P \longmapsto P^{\prime}$ and $\llbracket P^{\prime} \rrbracket \equiv Q$.

Proof: From Propositions 8 and 9.

\subsection{Observational Equivalence}

To complete our results, we managed to prove an observational equivalence property. This result is not really useful for the encoding in pure ambients, but a soundness result might also be interesting for future work.

The observability predicate $P \downarrow M$ is defined on $\pi$-processes in the usual way (for example, $n(x) . P \downarrow n$ ), and can be easily extended to $\pi_{e s c}$-processes (for variables, substitution must be performed, i.e. $(\nu x: M) P \downarrow M$ when $P \downarrow x)$.

For the $\pi$-calculus:

$$
\frac{P \downarrow M \quad n \neq M}{(\nu n) P \downarrow M} \text { (Obs Res) } \quad \frac{P \downarrow M}{P \mid Q \downarrow M} \text { (Obs ParL) }
$$




$$
\begin{aligned}
\frac{Q \downarrow M}{P \mid Q \downarrow M} \text { (Obs ParR) } & \frac{P \downarrow M}{! P \downarrow M} \text { (Obs Repl) } \\
\overline{\bar{M}\left\langle M^{\prime}\right\rangle . P \downarrow M} \text { (Obs Output) } & \frac{}{M(x) \cdot P \downarrow M} \text { (Obs Input) }
\end{aligned}
$$

For the $\pi_{e s c}$-calculus, we add:

$$
\begin{array}{cc}
\frac{S \not \equiv \varepsilon}{[n: S] \downarrow n} & \text { (Obs Channel) } \\
\frac{P \downarrow M \quad x \neq M}{\left(\nu x: M^{\prime}\right) P \downarrow M}\left(\mathrm{Obs}_{\left.\operatorname{Var}_{1}\right)}\right. & \frac{P \downarrow x}{(\nu x: M) P \downarrow M}\left(\mathrm{Obs}_{\left.\mathrm{Var}_{2}\right)}\right)
\end{array}
$$

Proposition 12 For a process $P$ in the $\pi_{e s c}$-calculus, $P \downarrow M \Leftrightarrow \llbracket P \rrbracket \downarrow M$.

Proof: See Section A.8.

Corollary 13 Let $P$ be a complete $\pi_{e s c}$-process. Then, we have $\llbracket P \rrbracket \longrightarrow^{*} \downarrow M$ if and only if $\varnothing: P \longmapsto * \downarrow M$.

Proof: By induction on the length of the reductions, with the help of Corollary 11 and Proposition 12.

\subsection{Soundness}

We conclude this first set of results with a soundness theorem between the $\pi$ and $\pi_{e s c}$-calculi.

First of all, we need to choose a suitable equivalence between processes for both of these calculi. For convenience, we will use barbed bisimulation. Here is the definition for the $\pi$-calculus:

Definition 14 A relation $\mathcal{R}$ is a barbed bisimulation for the $\pi$-calculus if, whenever $P \mathcal{R} Q$ for two $\pi$-processes $P$ and $Q$, we have:

- if $P \longrightarrow P^{\prime}$, there is a process $Q^{\prime}$ such that $Q \longrightarrow^{*} Q^{\prime}$ and $P^{\prime} \mathcal{R} Q^{\prime}$.

- if $Q \longrightarrow Q^{\prime}$, there is a process $P^{\prime}$ such that $P \longrightarrow^{*} P^{\prime}$ and $P^{\prime} \mathcal{R} Q^{\prime}$.

- $P \longrightarrow^{*} \downarrow M$ if and only if $Q \longrightarrow^{*} \downarrow M$.

Let

$$
\approx_{\pi}=\bigcup\{\mathcal{R} / \mathcal{R} \text { is a barbed bisimulation }\}
$$

One can check that $\approx_{\pi}$ is the largest barbed bisimulation and that it contains structural congruence $\equiv$ (these are classical results).

We now give a very similar definition for complete processes in the $\pi_{e s c}$-calculus:

INRIA 
Definition $15 A$ relation $\mathcal{R}$ is a barbed bisimulation for the $\pi_{\text {esc }}$-calculus if, whenever $P \mathcal{R} Q$ for two complete $\pi_{\text {esc }}$-processes $P$ and $Q$, we have:

- if $\varnothing: P \longmapsto P^{\prime}$, there is a process $Q^{\prime}$ such that $\varnothing: Q \longmapsto^{*} Q^{\prime}$ and $P^{\prime} \mathcal{R} Q^{\prime}$.

- if $\varnothing: Q \longmapsto Q^{\prime}$, there is a process $P^{\prime}$ such that $\varnothing: P \longmapsto{ }^{*} P^{\prime}$ and $P^{\prime} \mathcal{R} Q^{\prime}$.

- $\varnothing: P \longmapsto^{*} \downarrow M$ if and only if $\varnothing: Q \longmapsto^{*} \downarrow M$.

Let

$$
\approx_{e s c}=\bigcup\{\mathcal{R} / \mathcal{R} \text { is a barbed bisimulation }\}
$$

One can check that $\approx_{e s c}$ is the largest barbed bisimulation.

Finally, we can state the soundness result: the encodings of two equivalent processes are equivalent, and vice versa.

Theorem 16 (Soundness) Let $P$ and $Q$ be two complete $\pi_{e s c}$-processes. Then, $P \approx_{e s c} Q$ if and only if $\llbracket P \rrbracket \approx_{\pi} \llbracket Q \rrbracket$.

Proof: We prove the two implications separately.

$\mathbf{P} \approx_{\text {esc }} \mathbf{Q} \Rightarrow \llbracket \mathbf{P} \rrbracket \approx_{\pi} \llbracket \mathbf{Q} \rrbracket$ We define the relation $\mathcal{R}$ by $P \mathcal{R} Q$ when $P \equiv \llbracket P_{0} \rrbracket, Q \equiv \llbracket Q_{0} \rrbracket$ and $P_{0} \approx_{e s c} Q_{0}$. We need to show that $\mathcal{R}$ is a barbed bisimulation (then $\mathcal{R} \subseteq \approx_{\pi}$ ). To do this, suppose that $P \mathcal{R} Q$ (and consequently that such $P_{0}$ and $Q_{0}$ exist).

- Suppose that $P \longrightarrow P^{\prime}$. Then, $\llbracket P_{0} \rrbracket \longrightarrow P^{\prime}$. By Corollary 11, there is a process $P^{\prime \prime}$ such that $\varnothing: P_{0} \longmapsto^{+} P^{\prime \prime}$ and $\llbracket P^{\prime \prime} \rrbracket \equiv P^{\prime}$. Since $P_{0} \approx_{e s c} Q_{0}$, there is a process $Q^{\prime}$ such that $\varnothing: Q_{0} \longmapsto^{*} Q^{\prime}$ and $P^{\prime \prime} \approx_{e s c} Q^{\prime}$. Then, by Corollary 11, we have $Q \equiv \llbracket Q_{0} \rrbracket \longrightarrow^{*} \llbracket Q^{\prime} \rrbracket$. And, $P^{\prime} \mathcal{R} \llbracket Q^{\prime} \rrbracket$ using the definition of $\mathcal{R}$.

- The reasoning is similar when $Q$ reduces.

- Using Lemma 63, Corollary 13 and Definition 15 , we have the following equivalences: $P \longrightarrow^{*} \downarrow M$ if and only if $\llbracket P_{0} \rrbracket \longrightarrow^{*} \downarrow M$ if and only if $\varnothing: P_{0} \longmapsto^{*} \downarrow M$ if and only if $\varnothing: Q_{0} \longmapsto^{*} \downarrow M$ if and only if $\llbracket Q_{0} \rrbracket \longrightarrow^{*} \downarrow M$ if and only if $Q \longrightarrow^{*} \downarrow M$.

$\llbracket \mathbf{P} \rrbracket \approx_{\pi} \llbracket \mathbf{Q} \rrbracket \Rightarrow \mathbf{P} \approx_{\text {esc }} \mathbf{Q}$ We define the relation $\mathcal{R}$ by $P \mathcal{R} Q$ when $\llbracket P \rrbracket \approx_{\pi} \llbracket Q \rrbracket$. We need to show that $\mathcal{R}$ is a barbed bisimulation (then $\mathcal{R} \subseteq \approx_{e s c}$ ). To do this, suppose that $P \mathcal{R} Q$.

- Suppose that $\varnothing: P \longmapsto P^{\prime}$. By Corollary 11 , we have $\llbracket P \rrbracket \equiv \llbracket P^{\prime} \rrbracket$. There are two cases:

- If $\llbracket P^{\prime} \rrbracket \equiv \llbracket P \rrbracket$, then $\llbracket P^{\prime} \rrbracket \approx_{\pi} \llbracket P \rrbracket$ and $\llbracket P^{\prime} \rrbracket \approx_{\pi} \llbracket Q \rrbracket$ by transitivity of $\approx_{\pi}$. Finally, $P^{\prime} \mathcal{R} Q$.

- If $\llbracket P \rrbracket \longrightarrow \llbracket P^{\prime} \rrbracket$, since $\approx_{\pi}$ is a bisimulation, there is a process $Q^{\prime}$ such that $\llbracket Q \rrbracket \longrightarrow^{*} Q^{\prime}$ and $\llbracket P^{\prime} \rrbracket \approx_{\pi} Q^{\prime}$. By Corollary 11 , there is a process $Q^{\prime \prime}$ such that $\varnothing: Q \longmapsto^{*} Q^{\prime \prime}$ and $\llbracket Q^{\prime \prime} \rrbracket \equiv Q^{\prime}$. Then, $Q^{\prime} \approx_{\pi} \llbracket Q^{\prime \prime} \rrbracket$ and $\llbracket P^{\prime} \rrbracket \approx_{\pi} \llbracket Q^{\prime \prime} \rrbracket$ by transitivity of $\approx_{\pi}$. Finally, $P^{\prime} \mathcal{R} Q^{\prime \prime}$.

- The reasoning is similar when $Q$ reduces. 
- Using Corollary 13 and Definition 14, we have the following equivalences: $\varnothing: P \longmapsto{ }^{*} \downarrow$ $M$ if and only if $\llbracket P \rrbracket \longrightarrow^{*} \downarrow M$ if and only if $\llbracket Q \rrbracket \longrightarrow^{*} \downarrow M$ if and only if $\varnothing: Q \longmapsto{ }^{*}$ $\downarrow M$.

\subsection{From the $\pi$-calculus to the $\pi_{e s c}$-calculus}

There is a simple way to transform a $\pi$-process into a "correct" $\pi_{e s c}$-process: replace every construct $(\nu n) P$ with $(\nu n)([n: \varepsilon] \mid P)$ and add an empty channel for every free name of $P$. In fact, this is exactly the definition of the channel-closure $c l_{\varnothing}(P)$ (if we view the $\pi$-process $P$ as a $\pi_{e s c}$-process). It has the following interesting properties: $c l_{\varnothing}(P)$ is valid, channel-closed with regard to $\varnothing$ and has no free variables if $P$ has none (these properties allow us to use Corollary 11).

Proposition $17 \mathrm{cl}_{\varnothing}(P)$ is channel-closed with regard to $\varnothing$ and $\vdash c l_{\varnothing}(P):$ Valid. Moreover, if $f v(P)=\emptyset, f v\left(c l_{\varnothing}(P)\right)=\emptyset$. Consequently, $c_{\varnothing}(P)$ is complete if $P$ has no free variables.

\subsection{On the choice of the $\pi_{e s c}$-calculus}

Explicit channels and variables are similar in their structure, but we used different syntaxes: two constructs $(\nu n)$ and $[n: S]$ for channels, and the single construct $(\nu x: M)$ for variables. One may ask why we retained this combination. Now is the time to answer this question.

We could have chosen to separate variables into a restriction $(\nu x)$ and an explicit variable $[x: M]$, with rule $\left(\pi_{e s c}\right.$ Red Subst Out) being $\sigma:[x: M]\left|\bar{x}\left\langle M^{\prime}\right\rangle . P \longmapsto[x: M]\right| \bar{M}\left\langle M^{\prime}\right\rangle . P$ (and similarly for $\left(\pi_{e s c}\right.$ Red Subst In)). But in order to evaluate $\llbracket(\nu x) P \rrbracket$, we would have needed a way to reach the object $[x: M]$ in $P$ and get the value $M$. This would have led to a very long technical development.

On the other hand, we could have chosen to include the content of a channel in the restriction operator with $(\nu n: S)$. In this case, we get a restriction interference. For instance, the process $(\nu n: \varepsilon)(\nu x: n) \bar{n}\langle x\rangle . P$ should reduce by putting the concretion $\langle x\rangle . P$ into $n$, but neither $(\nu n:\langle x\rangle . P)(\nu x: n) \mathbf{0}$ nor $(\nu x: n)(\nu n:\langle x\rangle . P) \mathbf{0}$ would be correct: in each case, a bound name becomes free.

\section{Encoding the $\pi_{e s c}$-Calculus in Pure Ambients}

\subsection{The Encoding}

The main mechanism underlying the encoding of $\pi_{e s c}$ in pure ambients is a kind of communication based on the request/server model. In pure ambients, a request willing to communicate with $n$ is an ambient named $r w$ with the process request $r w n$ inside it (in our encoding, $r w$ will be only read or write). Its first movement is to enter $n$. Symmetrically, a server is a replicated ambient enter inside the destination $n$ which tries to enter the request 
and take its control. The underlying protocol is that, after the ambient-request has entered the ambient-server, the request accepts the server code and let it run inside him. This mechanism is similar to the encoding of objective moves of [Cardelli and Gordon, 1998]. Let us first define these useful abbreviations:

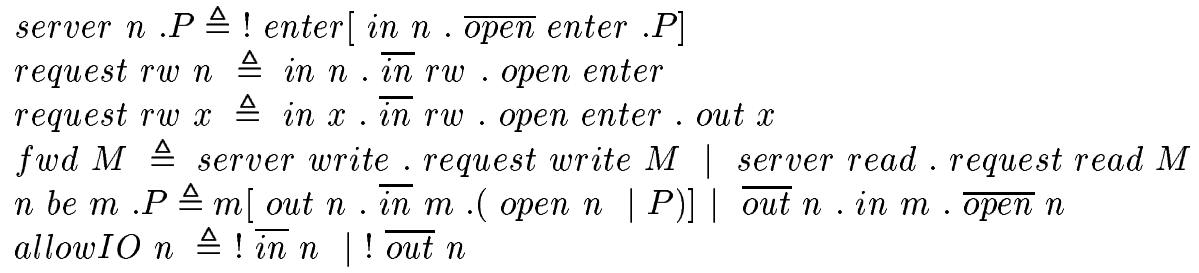

For example, here is the general interaction between a request and a server:

$$
\begin{aligned}
& n[\operatorname{server} r w . P \mid \text { allowIO } n] \mid r w[\text { request } r w n \mid Q] \\
\hookrightarrow^{+} \quad & n[\operatorname{server} r w . P \mid \text { allowIO } n \mid r w[P \mid Q]] \text { for } r w=\text { read or write }
\end{aligned}
$$

A variable $x$ whose value is $M$ is simply an ambient named $x$ with two servers inside it that replace every request with a similar request on $M$. Thus, a variable is simply a forwarder.

$$
\begin{aligned}
& \quad x[f w d M\mid \text { allowIO } x] \mid r w[\text { request } r w x \mid P] \\
& \hookrightarrow^{+} \quad x[f w d M \mid \text { allowIO } x] \mid r w[\text { request } r w M \quad \mid P] \text { for } r w=\text { read or } w r i t e
\end{aligned}
$$

A channel $n$ is simulated by an ambient named $n$ with a special server for read requests (there is no server for write requests). When $n$ contains a read request, it tries to find and take control of a write request (with always the same request/server mechanism). When this is done, the read request is replaced by an ambient $x$ whose content is the forwarder of the write request. Then, the two continuations are activated. Some intermediate ambient renamings are necessary to avoid interferences.

We do not detail the encoding further as it is not very instructive. We believe the only way to understand it fully is to test it by hand and try to mimick the reductions of the $\pi_{e s c}$-calculus. Some of these are given in Section A.9 in the proof of Proposition 18.

The full definition of the encoding is presented below.

$$
\begin{aligned}
& \{(\nu n) P\} \triangleq(\nu n)\{\{P\} \\
& \{0\} \triangleq \mathbf{0} \\
& \{\{P \mid Q\} \triangleq\{\{P\} \mid\{\{Q\}\} \\
& \{! P\} \triangleq !\{\{P\} \\
& \left\{\left[\bar{M}\left\langle M^{\prime}\right\rangle \cdot P\right\} \triangleq(\nu p) \quad \text { ( write [ request write } M\right. \\
& \text { f } w d M^{\prime} \\
& \text { | } p[\text { out read. open } p .\{\{P\}]] \\
& \text { | open } p \text { ) }
\end{aligned}
$$

$\mathrm{RR} \mathrm{n}^{\circ} 4350$ 


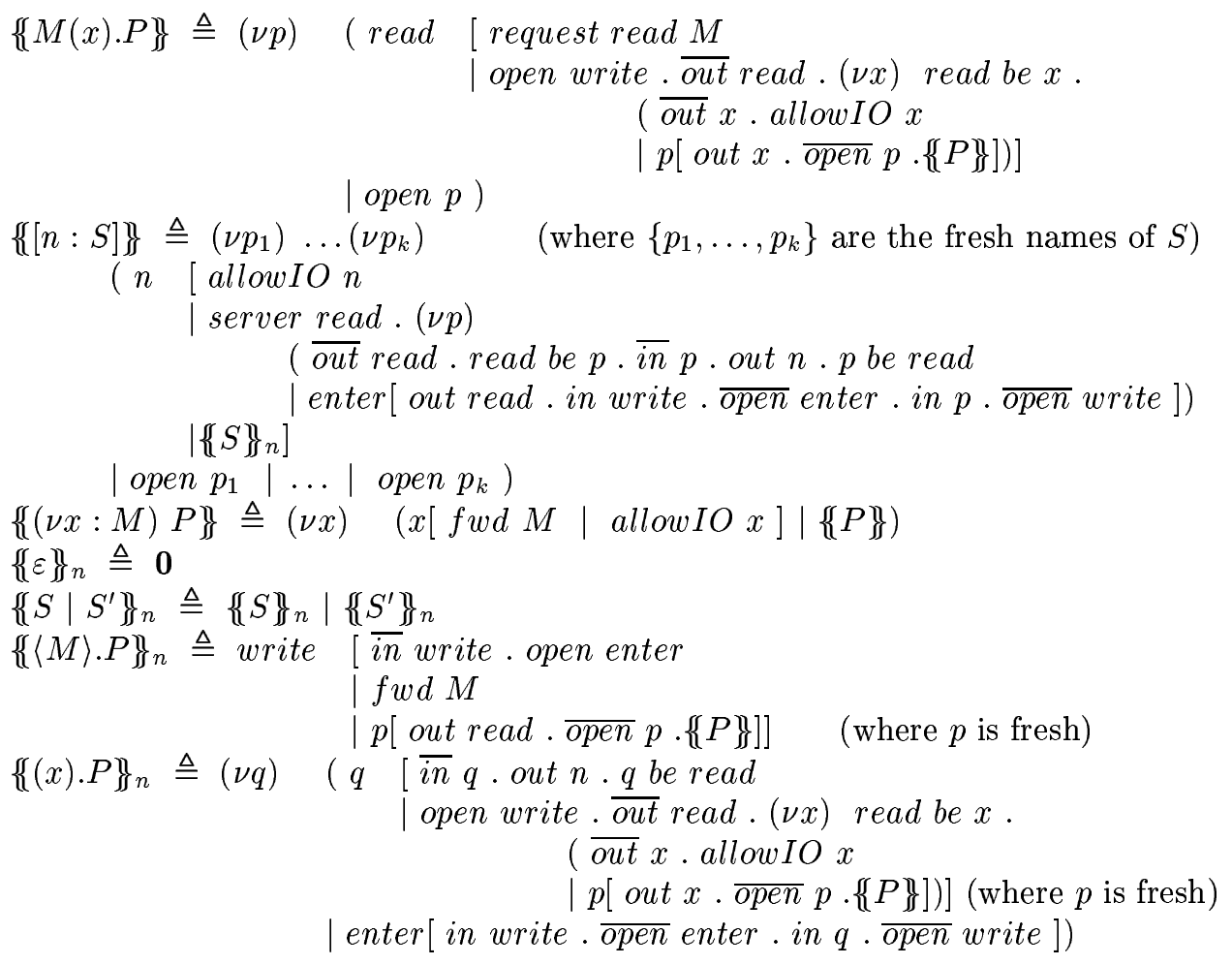

To handle substitutions, we add the following definition:

$$
\begin{aligned}
& \left\{\left\{{ }^{M_{1}} / x_{1}\right\} \uplus \ldots \uplus\left\{{ }^{M_{k}} / x_{k}\right\}, P\right\} \triangleq \quad x_{1}\left[\text { fwd } M_{1} \mid \text { allowIO } x_{1}\right] \\
& \text { | } \ldots \\
& x_{k}\left[\text { fwd } M_{k} \mid \text { allowIO } x_{k}\right] \\
& \{\{P\}
\end{aligned}
$$

\section{$5.2 \quad$ Results}

Before we state some properties, we need to distinguish two kinds of reductions in safe ambients. Principal reductions, written $\stackrel{p r}{\rightarrow}$, correspond intuitively to the first reductions of the encodings into pure ambients of the axiomatic reduction rules from the $\pi_{e s c}$-calculus. More precisely, we can pinpoint them by "marking" some specific capabilities in the encoding. These are the in $n$ and in $x$ capabilities in request $r w n$ and request $r w x$, and the in write capability in the ambient enter in $\{[n: S]\}$. Every reduction involving one of these marked capabilities are principal. All the others are auxiliary and are written $\stackrel{a u x}{\hookrightarrow}$.

Then, we can show that every reduction in the $\pi_{e s c}$-calculus corresponds to one principal followed by many auxiliary reductions in the encoding. 
Proposition 18 If $\sigma: P \longmapsto Q$, then $\{\sigma, P\} \stackrel{p r}{\leftrightarrow} \stackrel{a u x^{*}}{\hookrightarrow}\{\sigma \sigma, Q\}$.

Proof: By induction on the derivation of $\sigma: P \longmapsto Q$. Basically, we have to check that every reduction rule in the $\pi_{e s c}$-calculus is mimicked by several reductions in the encoding, which is technical routine. We just give one example here, please refer to Section A.9 for the other cases.

( $\pi_{e s c}$ Red Subst Out) Suppose that $\sigma: \bar{x}\left\langle M^{\prime}\right\rangle . P \longmapsto \bar{M}\left\langle M^{\prime}\right\rangle . P$ with $x \sigma=M$. To simplify, we consider only $\sigma=\left\{{ }^{M} / x\right\}$. It is not difficult to derive the general case with an arbitrary $\sigma$. We have (assuming that $p$ does not interfere with other names):

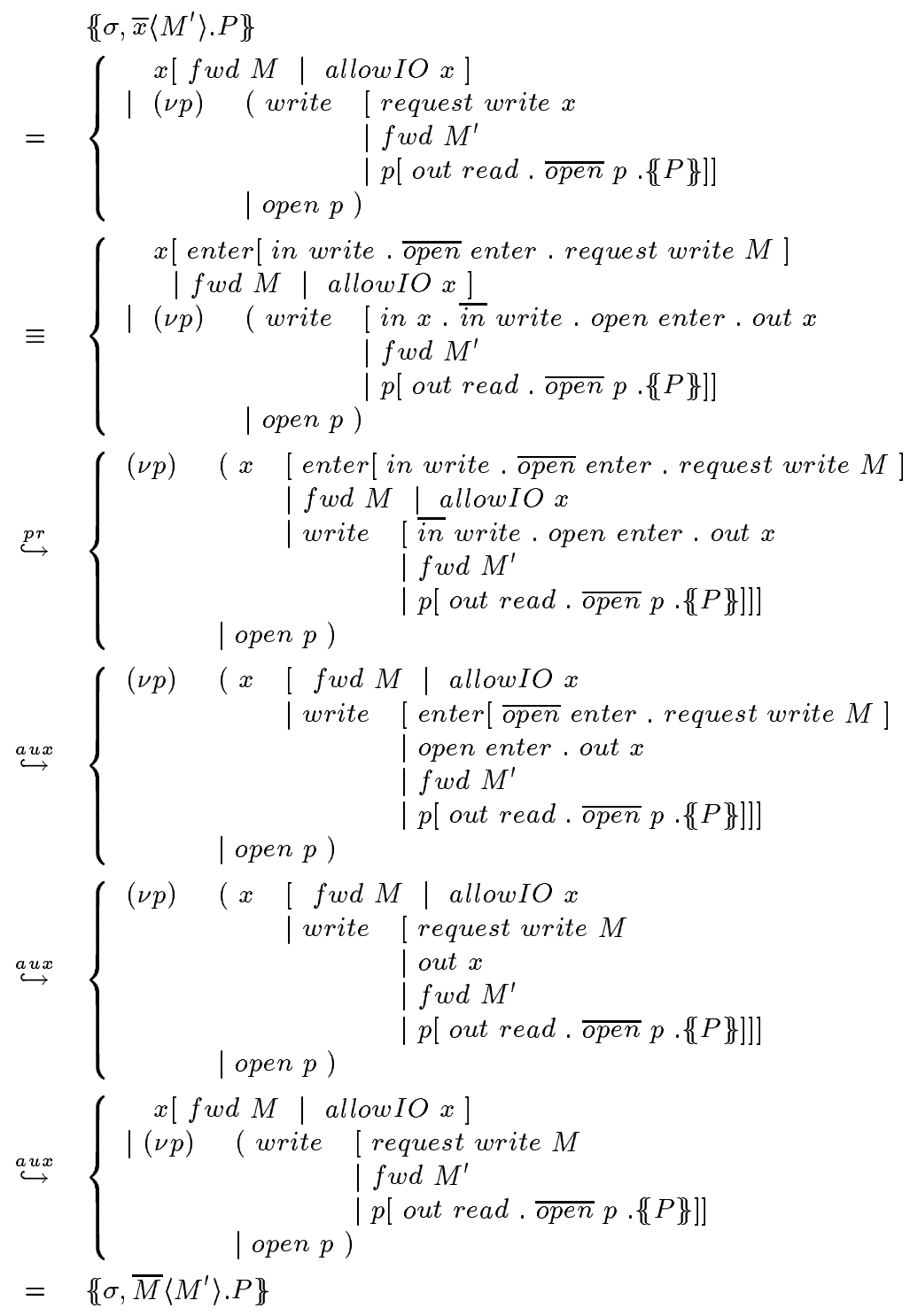


In the other direction, we can prove that if an encoded ambient term has a principal reduction, one can extend it with auxiliary reductions so that it corresponds to one single $\pi_{e s c}$-reduction. Moreover, this single reduction is unique in some sense, up to structural congruence.

Proposition 19 If $\left\{\{\sigma, P\} \stackrel{p r}{\hookrightarrow} Q\right.$, then there is a process $P^{\prime}$ such that $\sigma: P \longmapsto P^{\prime}$ and $Q \stackrel{a u x^{*}}{\hookrightarrow}\left\{\left\{\sigma, P^{\prime}\right\}\right.$. Moreover, if $\sigma: P \longmapsto P^{\prime \prime}$ and $Q \stackrel{a u x^{*}}{\hookrightarrow}\left\{\left\{\sigma, P^{\prime \prime}\right\}\right.$, then $P^{\prime} \equiv P^{\prime \prime}$.

Proof: Since $\{a, P\} \stackrel{p r}{\longrightarrow} Q$ is a principal reduction, it must involve one of the "marked" capabilities. This capability determines a corresponding axiomatic reduction in the $\pi_{e s c}$-calculus, namely:

- $\left(\pi_{e s c}\right.$ Red Subst Out) if the capability is in $x$ in request write $x$

- $\left(\pi_{e s c}\right.$ Red Subst In) if the capability is in $x$ in request read $x$

- $\left(\pi_{e s c}\right.$ Red Output) if the capability is in $n$ in request write $n$

- $\left(\pi_{e s c}\right.$ Red Input) if the capability is in $n$ in request read $n$

- $\left(\pi_{e s c}\right.$ Red Comm $)$ if the capability is in write in the ambient enter in $\{[n: S]\}$

Starting from this axiom, it is not difficult to determine the reduction $\sigma: P \longmapsto P^{\prime}$ in $\pi_{e s c}$. Then, with the same arguments as in the proof of Proposition 18, we can find the auxiliary reductions in $\pi_{e s c}$ such that $Q \stackrel{a u x^{*}}{\hookrightarrow}\left\{\sigma \sigma, P^{\prime}\right\}$.

Moreover, since the first principal reduction determines uniquely the corresponding axiom (and reduction) in $\pi_{e s c}$, the resulting process $P^{\prime}$ is unique modulo $\equiv$, which implies the second part of the proposition.

We need to explain why we have to distinguish between principal and auxiliary reductions. A counter-example, written in CCS style, is $P \triangleq ! a|! \bar{a}| b . C \mid \bar{b} . D$. We have $P \longrightarrow P$ and $P \longrightarrow P^{\prime}=! a|! \bar{a}| C \mid D$. Considering the first reduction, the last theorem would give $\{P\} \hookrightarrow Q$, with $P \longrightarrow P$ and $Q \hookrightarrow^{*}\{\{P\}$. But we also have $P \longrightarrow P^{\prime}$ and $Q \hookrightarrow^{*}\left\{\left\{P^{\prime}\right\}\right.$, with $P \not \equiv P^{\prime}$. Thus the second assertion would be false. The problem is avoided by distinguishing the two kinds of reductions: there must be a principal reduction between $Q$ and $\left\{\left\{P^{\prime}\right\}\right.$.

However, Proposition 19 is not as strong as we would hope: we always reach the next encoded term with auxiliary reductions before the next principal reduction. In fact, auxiliary reductions do not really matter: our encoding was designed so that a new effective step in the computation (i.e. a principal reduction) can take place as soon as possible (sometimes a few auxiliary reductions are needed before to unblock the situation). This is why we believe the following conjecture to be true. Proving it is not difficult in theory, but we face a huge number of cases to examine, leading to a combinatorial explosion that only an automatic demonstration tool could maybe handle.

Conjecture $20 \stackrel{\text { aux }}{\hookrightarrow}$ is confluent with $\stackrel{a u x}{\hookrightarrow}$ and $\stackrel{p r}{\hookrightarrow}$, that is:

- if $P \stackrel{a u x}{\hookrightarrow} P_{1}$ and $P \stackrel{\text { aux }}{\hookrightarrow} P_{2}$, there is a process $Q$ such that $P_{1} \stackrel{a u x}{\hookrightarrow} Q$ and $P_{2} \stackrel{\text { aux }}{\hookrightarrow} Q$.

- if $P \stackrel{p r}{\hookrightarrow} P_{1}$ and $P \stackrel{a u x}{\hookrightarrow} P_{2}$, there is a process $Q$ such that $P_{1} \stackrel{a u x}{\hookrightarrow} Q$ and $P_{2} \stackrel{p r}{\hookrightarrow} Q$. 


\subsection{Observational Equivalence}

As we did for the $\pi_{e s c}$-calculus, we are able to define an observation predicate, and show an adequacy result: observability is preserved by the encoding.

However, observability in pure ambients will be more difficult to define and well-adapted to the encoding, by means of evaluation contexts:

Definition 21 An evaluation context is a context where the hole [.] occurs only once and not under a guard. More precisely, they are of the form: $C[\cdot] \triangleq(\nu \vec{n})([\cdot] \mid P)$.

Definition 22 The observation predicate is defined in pure ambients by: $P \downarrow M$ if and only if either $\left.P \equiv C\left[\left\{\bar{M}\left\langle M^{\prime}\right\rangle \cdot Q\right\}\right\}\right]$ or $P \equiv C[\{M(x) \cdot Q\}]$ or $P \equiv C[\{[M: S]\}]$ for some evaluation context $C[\cdot]$ that does not bind $M$, some process $Q$, some names $M$ and $M^{\prime}$, and some term $S \not \equiv \varepsilon$ (depending on the case, not all of these conditions are needed).

With this definition, we can state the adequacy property:

Proposition 23 For a $\pi_{e s c}$-process $P$, we have:

- $P \downarrow M \Rightarrow\left\{\{P\} \hookrightarrow^{*} \downarrow M\right.$

- $\{\{P\} \downarrow M \Rightarrow P \downarrow M$

\section{Proof:}

- By induction on the derivation of $P \downarrow M$. The only non-trivial case is for (Obs $\left.\operatorname{Var}_{2}\right)$ : when $(\nu x: M) P \downarrow M$ has been derived from $P \downarrow x$. Then, by induction hypothesis, we get $\left\{\{P\} \hookrightarrow^{*} Q \downarrow x\right.$. We have:

$$
\begin{array}{rllll}
\{(\nu x: M) P\} & =(\nu x)(x[\text { fwd } M & \text { allowIO } x] \mid\{\{P\}) \\
& \hookrightarrow^{*} & (\nu x)(x[\text { fwd } M & \text { allowIO } x] \mid Q)
\end{array}
$$

Since $Q \downarrow x$, necessarily $Q \equiv C\left[\left\{\bar{x}\left\langle M^{\prime}\right\rangle \cdot Q^{\prime}\right\}\right]$ or $Q \equiv C\left[\left\{x(y) \cdot Q^{\prime}\right\}\right]$ for some evaluation context $C[\cdot]$ that does not bind $x$ nor $M$ (since the oberved name $x$ is a variable, we cannot be in the third case, i.e. of a non-empty channel). Then (supposing we are in the first case),

$$
\begin{array}{rll}
\{\{(\nu x: M) P\} & \hookrightarrow^{*} & (\nu x)\left(x[\text { fwd } M \mid \text { allowIO } x] \mid C\left[\left\{\bar{x}\left\langle M^{\prime}\right\rangle \cdot Q^{\prime}\right\}\right]\right) \\
& \equiv & C\left[(\nu x)\left(x[\text { fwd } M \mid \text { allowIO } x] \mid\left\{\left[\bar{x}\left\langle M^{\prime}\right\rangle \cdot Q^{\prime}\right\}\right)\right]\right. \\
& \hookrightarrow^{+} & R=C\left[(\nu x)\left(x[\text { fwd } M \mid \text { allowIO } x] \mid\left\{\bar{M}\left\langle M^{\prime}\right\rangle \cdot Q^{\prime}\right\}\right)\right]
\end{array}
$$

and we finally get: $R \downarrow M$.

- By direct and easy induction on $P$.

Soundness (discussion): With such an observation predicate, is it possible to define some equivalence in pure ambients (barbed bisimulation or some other), and show a soundness property as we did for the encoding between $\pi$ and $\pi_{e s c}$-calculi ? We are 
not sure if such a result would be technically reachable, but what is more is that we are not really interested in it... The resulting equivalence in pure ambients would seem too artificial to us, since the observation predicate is really too specific to our encoding and very different from classical definitions of observability in ambients (for example, observing the name $n$ for every ambient $n[P])$. Thus, such an equivalence would be very poor from our point of view...

\section{The Final Encoding}

It remains to compose the results of the two previous Sections. The encoding of a $\pi$-process $P$ into pure ambients is defined by:

$$
\langle\langle P\rangle\rangle \triangleq\left\{\left\{\varnothing, c l_{\varnothing}(P)\right\}\right.
$$

Using the definitions in Section 5, we can give the final encoding directly, and not via the $\pi_{e s c}$-calculus. Those definitions apply to processes without free names; otherwise we need to add an empty channel for each free name (this implies that, strictly speaking, the encoding is fully compositional only for processes without free names):

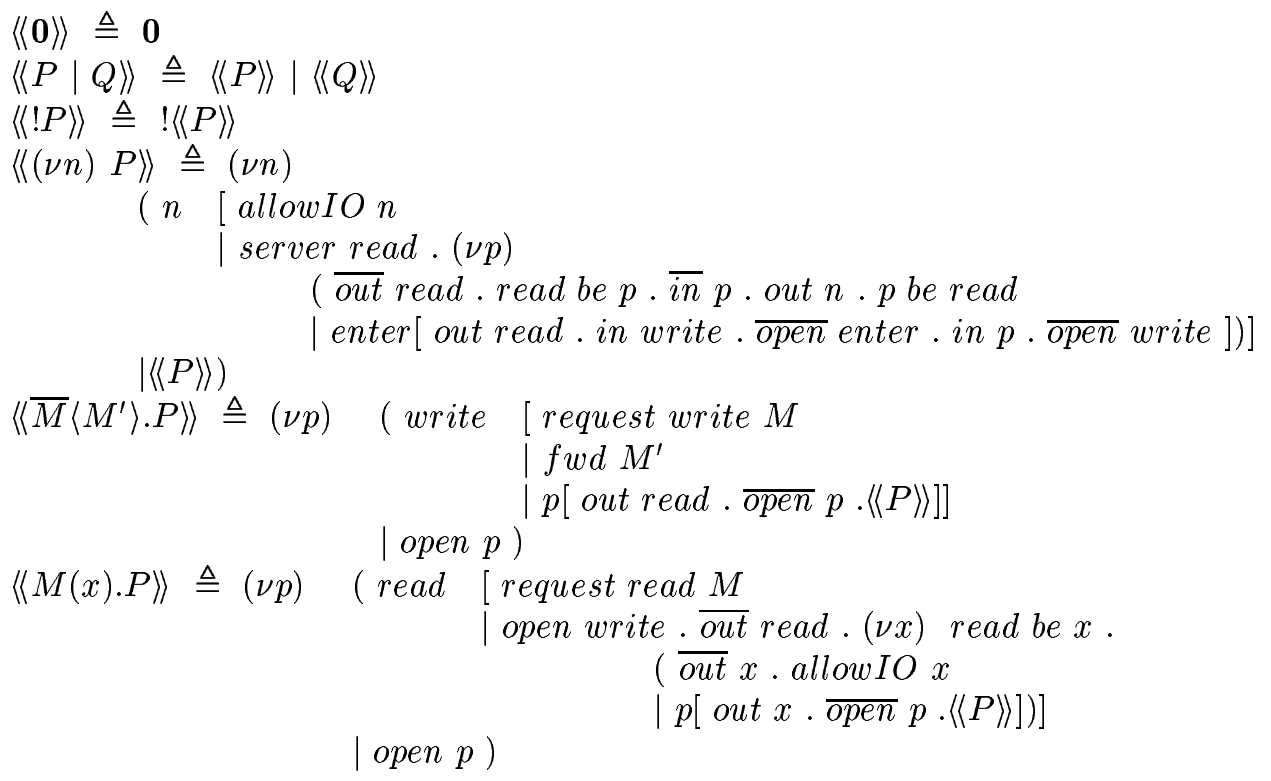

It remains to state some operational correspondence properties. We first define an equivalence relation $\bowtie$ between the $\pi$-calculus and pure ambients.

Definition 24 Let $P$ be a $\pi$-process with no free variables and $R$ a pure ambient process. We say that $P$ and $R$ are equivalent (written $P \bowtie R$ ) if there is a complete $\pi_{\text {esc }}$-process $Q$ such that $P \equiv \llbracket Q \rrbracket$ and $\{\varnothing \varnothing, Q\} \equiv R$. 
It is routine to check that $P \bowtie\langle\langle P\rangle\rangle$ for every $\pi$-process $P$ with no free variables.

With this definition, we can state the final operational correspondence theorem:

Theorem 25 Suppose $P \bowtie R$.

- If $P \longrightarrow P^{\prime}$, then there is a process $R^{\prime}$ such that $R \hookrightarrow^{+} R^{\prime}$ and $P^{\prime} \bowtie R^{\prime}$.

- If $R \stackrel{p r}{\rightarrow} R^{\prime}$, then there is a process $R^{\prime \prime}$ such that $R^{\prime} \stackrel{\text { aux* }}{\longrightarrow} R^{\prime \prime}$ and either $P \bowtie R^{\prime \prime}$, or $P \longrightarrow P^{\prime} \bowtie R^{\prime \prime}$.

- $P \downarrow M \Rightarrow R \hookrightarrow^{*} \downarrow M$ and $R \downarrow M \Rightarrow P \downarrow M$.

Proof: Combine Corollary 11 and Propositions 18, 19, 12 and 23.

\section{Conclusion and Future Work}

We gave an encoding of the synchronous $\pi$-calculus into the ambient calculus with neither communication primitives nor substitutions. And we proved an operational correspondence for our encoding, showing that pure ambients are as expressive as the $\pi$-calculus. To do this, we designed the $\pi_{e s c}$-calculus in order to facilitate the proof. This calculus seems interesting in itself, since it models substitutions and channels explicitly. Independently from the encoding in pure ambients, we proved expressiveness and adequacy results between $\pi$ and $\pi_{e s c}$.

The first future work should be to use an automatic demonstration tool to prove Conjecture 20. If it succeeds, we could state a much stronger final theorem for our operational correspondence (namely that only principal reductions do really matter). Moreover, our encoding was also designed to avoid all interferences with other processes (if we restrict internal names for the request/server mechanism). Thus, we would like to show that no attack against

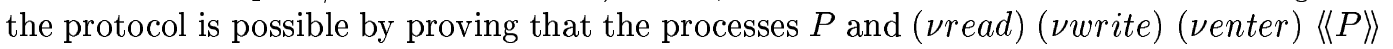
are "equivalent" in some sense.

Furthermore, a few expressiveness questions arise from our work. Is it possible to encode the $\pi$-calculus with classical mobile ambients instead of safe ambients (we already explained in the introduction why it seems difficult)? And more important to us: is it possible to encode the full ambient calculus (safe or not) with its communication primitives into the same calculus without communication primitives (in fact, this is the question which led us to do this work)? The main difference with the encoding of the $\pi$-calculus is that variables should now be present at every level in the hierarchy of ambients and not only at the global level. Thus, intuitively, they should replicate themselves and scatter dynamically, even in newly created ambients, and it is not obvious how to achieve this effect.

\section{Acknowledgements}

I benefited from many discussions with Davide Sangiorgi for this work. Thanks also to Ilaria Castellani, Gérard Boudol, Kohei Honda and Xudong Guan.

$\mathrm{RR} \mathrm{n}^{\circ} 4350$ 


\section{A Proofs}

\section{A.1 Lemmas Concerning Substitutions}

These first elementary lemmas deal with our special notion of substitution. They detail how commutativity, transitive closure, structural congruence and reduction relate to each other, and will be useful in many other proofs.

Lemma 26 If $x \notin \operatorname{dom}(\sigma),\left\{{ }^{M} / x\right\} \sigma=\sigma\left\{{ }^{M \sigma} / x\right\}$ and $\left\{{ }^{M} / x\right\} \sigma^{*}=\sigma^{*}\left\{{ }^{M \sigma^{*}} / x\right\}$

\section{Proof:}

- $x\left\{{ }^{M} / x\right\} \sigma=M \sigma=x\left\{{ }^{M \sigma} / x\right\}=x \sigma\left\{{ }^{M \sigma} / x\right\}$ since $x \notin \operatorname{dom}(\sigma)$. For $y \neq x, y\left\{{ }^{M} / x\right\} \sigma=y \sigma=$ $y \sigma\left\{{ }^{M \sigma} / x\right\}$ since $y \sigma \neq x$ (because $x \in i m(\sigma)$ implies $x \in \operatorname{dom}(\sigma)$ ).

- With $\sigma^{*}=\sigma^{p}$, we just need to apply the last result $p$ times.

Lemma 27 If $x \notin \operatorname{dom}(\sigma)$ and $M \in \operatorname{Name} \cup \operatorname{dom}(\sigma)$, then $\left(\left\{{ }^{M} / x\right\} \uplus \sigma\right)^{*}=\left\{{ }^{M} / x\right\} \sigma^{*}$.

Proof: We cannot have $M=x$, and since $x \notin \operatorname{dom}(\sigma), x \notin i m(\sigma)$. Thus, $x \notin i m\left(\left\{{ }^{M} / x\right\} \uplus \sigma\right)$. Consequently, for $M^{\prime} \neq x, M^{\prime}\left(\left\{{ }^{M} / x\right\} \uplus \sigma\right)^{*}=M^{\prime} \sigma^{*}=M^{\prime}\left\{{ }^{M} / x\right\} \sigma^{*}$. And $x\left(\left\{{ }^{M} / x\right\} \uplus \sigma\right)^{*}=$ $M\left(\left\{{ }^{M} / x\right\} \uplus \sigma\right)^{*}=M \sigma^{*}=x\left\{{ }^{M} / x\right\} \sigma^{*}$. Finally, $\left(\left\{{ }^{M} / x\right\} \uplus \sigma\right)^{*}=\left\{{ }^{M} / x\right\} \sigma^{*}$.

\section{Lemma 28}

- If $P \equiv Q$, then $P \sigma \equiv Q \sigma$.

- If $P \longrightarrow Q$, then $P \sigma \longrightarrow Q \sigma$.

Proof: By induction on the derivations of $P \equiv Q$ and $P \longrightarrow Q$.

Lemma 29 If $P \sigma \equiv Q$, then there exists $P^{\prime}$ such that $P \equiv P^{\prime}$ and $Q=P^{\prime} \sigma$.

Proof: By induction on the derivation of $P \sigma \equiv Q$. 


\section{A.2 Elementary Lemmas for the $\pi_{e s c}$-Calculus}

This Section contains some elementary lemmas for the $\pi_{e s c}$-calculus which will be useful in the final proofs.

\section{A.2.1 Free Names and Free Variables}

This lemma shows how free names and variables are preserved by structural congruence and reduction.

\section{Lemma 30}

- If $P \equiv Q$, then $f n(P)=f n(Q)$ and $f v(P)=f v(Q)$.

- If $\sigma: P \longmapsto Q$, then $f n(P) \cup f n(\sigma)=f n(Q) \cup f n(\sigma)$ and $f v(Q) \subseteq \operatorname{dom}(\sigma)$.

Proof: By induction on the derivations of $P \equiv Q$ and $\sigma: P \longmapsto Q$.

\section{A.2.2 Channel Presentation and Channel Closure}

The two following results state some basic facts.

Lemma $31 P \Downarrow_{2} n \Rightarrow P \Downarrow_{1} n \Rightarrow n \in f n(P)$

Proof: By induction on the derivations of $P \Downarrow_{2} n$ and $P \Downarrow_{1} n$.

Proposition $3 \operatorname{pr}(P) \subseteq f n(P)$

Proof: Using Lemma 31.

Channel presentation and channel closure are preserved by structural congruence.

Lemma 32 If $P \equiv Q$, then

- $P \Downarrow_{1} n \Leftrightarrow Q \Downarrow_{1} n$ for all $n \in$ Name

- $P \Downarrow_{2} n \Leftrightarrow Q \Downarrow_{2} n$ for all $n \in$ Name

- $\operatorname{cl}(P) \equiv \operatorname{cl}(Q)$

Proof: By induction on the derivation of $P \equiv Q$.

Corollary 33 If $P \equiv Q$, then $\operatorname{pr}(P)=\operatorname{pr}(Q)$ and $c l_{\sigma}(P) \equiv c l_{\sigma}(Q)$.

Proof: Using Lemma 30 and Lemma 32.

And channel presentation and channel closure are preserved by reduction in the $\pi_{e s c^{-}}$ calculus.

$\mathrm{RR} \mathrm{n}^{\circ} 4350$ 
Lemma 34 If $\sigma: P \longmapsto Q$,

- $P \Downarrow_{1} n \Leftrightarrow Q \Downarrow_{1} n$ for all $n \in$ Name

- $P \Downarrow_{2} n \Leftrightarrow Q \Downarrow_{2} n$ for all $n \in$ Name

- $\sigma: \operatorname{cl}(P) \longmapsto \operatorname{cl}(Q)$

Proof: By induction on the derivation of $\sigma: P \longmapsto Q$, using Lemma 32 .

Corollary 35 If $\sigma: P \longmapsto Q$,

- $\operatorname{pr}(P)=\operatorname{pr}(Q)$

- $\sigma: c l_{\sigma}(P) \longmapsto c l_{\sigma}(Q)$

- $P$ channel-closed with regard to $\sigma \Leftrightarrow Q$ channel-closed with regard to $\sigma$

Proof: Using Lemma 30 and Lemma 34.

A few more results about channel closure:

\section{Lemma 36}

- $f v(c l(P))=f v(P)$

- $P \Downarrow_{2} n$ if and only if $\operatorname{cl}(P) \Downarrow_{2} n$.

- $\vdash P$ : Valid if and only if $\vdash \operatorname{cl}(P):$ Valid.

Proof: By induction on $P$.

\section{A.2.3 Validity}

This Section gives the proof of Subject Reduction for the validity type system in $\pi_{e s c^{-}}$ calculus.

Lemma 37 If $P \equiv Q$, then $\vdash P:$ Valid $\Leftrightarrow \vdash Q:$ Valid.

Proof: We first prove $\vdash P: O K \Leftrightarrow \vdash Q: O K$ by induction on the derivation of $P \equiv Q$, using Lemma 31 and Lemma 32. Then, it is easy to prove $\vdash P:$ Valid $\Leftrightarrow \vdash Q:$ Valid with Lemma 32.

Proposition 6 If $\sigma: P \longmapsto Q$ and $\vdash P:$ Valid, then $\vdash Q:$ Valid.

Proof: We first prove $\vdash P: O K \Rightarrow \vdash Q: O K$ by induction on the derivation of $\sigma: P \longmapsto Q$, using Lemma 34 and Lemma 37. Then, it is easy to prove $\vdash P:$ Valid $\Rightarrow \vdash Q:$ Valid with Lemma 34 .

INRIA 


\section{A.2.4 Reductions}

This lemma details one elementary step of substitution.

Lemma 38 If $M \sigma=M^{\prime}$, then $\sigma: \bar{M}\left\langle M^{\prime \prime}\right\rangle . P \longmapsto^{*} \overline{M^{\prime}}\left\langle M^{\prime \prime}\right\rangle . P$ (provided that $f v\left(\bar{M}\left\langle M^{\prime \prime}\right\rangle . P\right) \subseteq$ $\operatorname{dom}(\sigma))$ and $\sigma: M(x) . P \longmapsto^{*} M^{\prime}(x) . P$ (provided that $f v(M(x) . P) \subseteq \operatorname{dom}(\sigma)$ ). The same holds if $M \sigma^{*}=M^{\prime}$.

Proof: If $M \notin \operatorname{dom}(\sigma)$, then $\bar{M}\left\langle M^{\prime \prime}\right\rangle \cdot P=\overline{M^{\prime}}\left\langle M^{\prime \prime}\right\rangle \cdot P$ and $M(x) \cdot P=M^{\prime}(x) . P$ with no reduction. Otherwise, $M=x$ for some variable name $x$, and $x \sigma=M^{\prime}$. Then, $\sigma: \bar{M}\left\langle M^{\prime \prime}\right\rangle . P \longmapsto \overline{M^{\prime}}\left\langle M^{\prime \prime}\right\rangle \cdot P$ by ( $\pi_{e s c}$ Red Subst Out) and $\sigma: M(x) . P \longmapsto M^{\prime}(x) . P$ by $\left(\pi_{e s c}\right.$ Red Subst In). If $M \sigma^{*}=M^{\prime}$, we just apply the last result $p$ times where $p$ is such that $\sigma^{*}=\sigma^{p}$ (there are at most $p$ reductions).

This lemma shows how we can extend environments without affecting reductions.

Lemma 39 If $x \notin \operatorname{dom}(\sigma), M \in N a m e \cup \operatorname{dom}(\sigma)$ and $\sigma: P \longmapsto P^{\prime}$, then $\left\{{ }^{M} / x\right\} \uplus \sigma$ : $P \longmapsto P^{\prime}$.

Proof: By induction on the derivation of $\sigma: P \longmapsto P^{\prime}$.

\section{A.2.5 Translation $\llbracket . \rrbracket$}

The following three lemmas show how the translation $\llbracket . \rrbracket$ relates to free names and variables, channel closure, and structural congruence.

Lemma $40 f n(\llbracket P \rrbracket) \subseteq f n(P)$ and $f v(\llbracket P \rrbracket) \subseteq f v(P)$.

Proof: By induction on the structure of $P$ (the inequalities come from the cases $[n: \varepsilon]$ and $(\nu x: M) P$ with $x \notin f v(\llbracket P \rrbracket))$.

Lemma $41 \llbracket c l(P) \rrbracket \equiv \llbracket P \rrbracket$

Proof: By induction on the structure of $P$.

Lemma 42 If $P \equiv Q$, then $\llbracket P \rrbracket \equiv \llbracket Q \rrbracket$.

Proof: By induction on the derivation of $P \equiv Q$.

( $\pi_{e s c}$ Struct Var) Suppose that $(\nu x: M) P \equiv(\nu x: M) Q$ was derived from $P \equiv Q$. By induction hypothesis, $\llbracket P \rrbracket \equiv \llbracket Q \rrbracket$. Then, $\llbracket P \rrbracket\left\{{ }^{M} / x\right\} \equiv \llbracket Q \rrbracket\left\{{ }^{M} / x\right\}$ by Lemma 28. Finally, $\llbracket(\nu x: M) P \rrbracket \equiv \llbracket(\nu x: M) Q \rrbracket$.

( $\pi_{\text {esc }}$ Struct Res Par) Suppose that $(\nu n)(P \mid Q) \equiv P \mid(\nu n) Q$ with $n \notin f n(P)$. By Lemma 40, $n \notin f n(\llbracket P \rrbracket)$. Then, we can derive $\llbracket(\nu n)(P \mid Q) \rrbracket=(\nu n)(\llbracket P \rrbracket \mid \llbracket Q \rrbracket) \equiv \llbracket P \rrbracket \mid(\nu n) \llbracket Q \rrbracket=$ $\llbracket P \mid(\nu n) Q \rrbracket$ with $(\pi$ Struct Res Par).

$\mathrm{RR} \mathrm{n}^{\circ} 4350$ 
( $\pi_{e s c}$ Struct Var Par) Suppose that $(\nu x: M)(P \mid Q) \equiv P \mid(\nu x: M) Q$ with $x \notin f v(P)$. By Lemma $40, x \notin f v(\llbracket P \rrbracket)$ and thus $\llbracket P \rrbracket\left\{{ }^{M} / x\right\}=\llbracket P \rrbracket$. Then, we have $\llbracket(\nu x: M)(P \mid Q) \rrbracket=$ $\llbracket P \rrbracket\left\{{ }^{M} / x\right\}\left|\llbracket Q \rrbracket\left\{{ }^{M} / x\right\}=\llbracket P \rrbracket\right| \llbracket(\nu x: M) Q \rrbracket=\llbracket P \mid(\nu x: M) Q \rrbracket$.

( $\pi_{e s c}$ Struct Res Var) Suppose that $(\nu n)(\nu x: M) P \equiv(\nu x: M)(\nu n) P$ with $n \neq M$. Then, $\llbracket(\nu n)(\nu x: M) P \rrbracket=(\nu n)\left(\llbracket P \rrbracket\left\{{ }^{M} / x\right\}\right)=((\nu n) \llbracket P \rrbracket)\left\{{ }^{M} / x\right\}=\llbracket(\nu x: M)(\nu n) P \rrbracket$ (the second equality is correct because $n \neq M)$.

$\left(\pi_{e s c}\right.$ Struct Var Var) Suppose $(\nu x: M)\left(\nu y: M^{\prime}\right) P \equiv\left(\nu y: M^{\prime}\right)(\nu x: M) P$ with $x \neq y, x \neq$ $M^{\prime}$ and $y \neq M$. With these conditions and Lemma $26,\left\{{ }^{M} / x\right\}\left\{{ }^{M^{\prime}} / y\right\}=\left\{{ }^{M^{\prime}} / y\right\}\left\{{ }^{M}\left\{{ }^{M^{\prime}} / y\right\} / x\right\}=$ $\left\{{ }^{M^{\prime}} / y\right\}\left\{{ }^{M} / x\right\}$. Then, $\llbracket(\nu x: M)\left(\nu y: M^{\prime}\right) P \rrbracket=\llbracket\left(\nu y: M^{\prime}\right)(\nu x: M) P \rrbracket$.

The other cases are trivial.

The next two results are decomposition lemmas when the translation of a process is either a parallel composition, or an input/output operator.

Lemma 43 If $\llbracket P \rrbracket=P_{1} \mid P_{2}$ and $P$ is not of the form $(\nu x: M) P^{\prime}$, then either

- $P=\left[n: S \mid S^{\prime}\right]$ with $\llbracket S \rrbracket_{n}=P_{1}$ and $\llbracket S^{\prime} \rrbracket_{n}=P_{2}$

- $P=P_{1}^{\prime} \mid P_{2}^{\prime}$ with $\llbracket P_{1}^{\prime} \rrbracket=P_{1}$ and $\llbracket P_{2}^{\prime} \rrbracket=P_{2}$

Proof: These are the only ways to derive $\llbracket P \rrbracket=P_{1} \mid P_{2}$.

Lemma 44 If $\llbracket P \rrbracket=\bar{M}\left\langle M^{\prime}\right\rangle \cdot P^{\prime}$ and $P$ is not of the form $\left(\nu x: M^{\prime \prime}\right) P^{\prime \prime}$, then either

- $P=\left[n:\left\langle M^{\prime}\right\rangle \cdot P^{\prime \prime}\right]$ with $M=n$ and $\llbracket P^{\prime \prime} \rrbracket=P^{\prime}$

- $P=\bar{M}\left\langle M^{\prime}\right\rangle \cdot P^{\prime \prime}$ with $\llbracket P^{\prime \prime} \rrbracket=P^{\prime}$

A similar lemma holds if $\llbracket P \rrbracket=M(x) \cdot P^{\prime}$.

Proof: These are the only ways to derive $\llbracket P \rrbracket=\bar{M}\left\langle M^{\prime}\right\rangle \cdot P^{\prime}$. 


\section{A.3 Lemmas for Channel Closure}

In this Section, we treat our first problem: channel closure does not mix well with an inductive proof. For this reason, we need a preliminary result for almost every construct, showing how channel closure behaves with them. We start here with the two easier cases: variable restriction and name restriction. The other cases need further technical development and will be treated in the following Sections. Furthermore, Lemma 46 gives a similar result in a special case.

Lemma $45(\nu x: M) c l_{\{M / x\} \uplus \sigma}(P) \equiv c l_{\sigma}((\nu x: M) P)$ if $x \notin \operatorname{dom}(\sigma)$ and $M \in$ Name $U$ $\operatorname{dom}(\sigma)$.

Proof: We have $\operatorname{pr}(P)=\operatorname{pr}((\nu x: M) P)$. Then, $\left(f n(P) \cup f n\left(\left\{{ }^{M} / x\right\} \uplus \sigma\right)\right) \backslash \operatorname{pr}(P)=$ $(f n(P) \cup f n(M) \cup f n(\sigma)) \backslash p r(P)=(f n((\nu x: M) P) \cup f n(\sigma)) \backslash p r((\nu x: M) P)$. Finally, $(\nu x: M) c l_{\{M / x\} \uplus \sigma}(P) \equiv(\nu x: M)\left(\left[n_{1}: \varepsilon\right]|\ldots|\left[n_{k}: \varepsilon\right] \mid c l(P)\right) \equiv\left[n_{1}: \varepsilon\right]|\ldots|\left[n_{k}:\right.$ $\varepsilon] \mid(\nu x: M) c l(P) \equiv c l_{\sigma}((\nu x: M) P)$, using $\left(\pi_{e s c}\right.$ Struct Var Par).

Lemma $46 \operatorname{cl}_{\sigma}(P) \equiv c l_{\sigma}\left(\left[n_{1}: \varepsilon\right]|\ldots|\left[n_{k}: \varepsilon\right] \mid P\right)$ if $n_{i} \in f n(P) \backslash p r(P)$.

Proof: Since $n_{i} \in f n(P) \backslash p r(P)$, we can write the set $(f n(P) \cup f n(\sigma)) \backslash p r(P)$ as $\left\{n_{1}, \ldots, n_{k}, m_{1}, \ldots m_{p}\right\}$. We can show $\operatorname{pr}\left(\left[n_{1}: \varepsilon\right]|\ldots|\left[n_{k}: \varepsilon\right] \mid P\right)=\left\{n_{1}, \ldots, n_{k}\right\} \cup \operatorname{pr}(P)$. Then, $\left(f n\left(\left[n_{1}: \varepsilon\right]|\ldots|\left[n_{k}:\right.\right.\right.$ $\varepsilon] \mid P) \cup f n(\sigma)) \backslash p r\left(\left[n_{1}: \varepsilon\right]|\ldots|\left[n_{k}: \varepsilon\right] \mid P\right)=\left(\left\{n_{1}, \ldots, n_{k}\right\} \cup f n(P) \cup f n(\sigma)\right) \backslash\left(\left\{n_{1}, \ldots, n_{k}\right\} \cup\right.$ $\operatorname{pr}(P))=((f n(P) \cup f n(\sigma)) \backslash p r(P)) \backslash\left\{n_{1}, \ldots, n_{k}\right\}=\left\{m_{1}, \ldots m_{p}\right\}$. Finally, $c l_{\sigma}(P) \equiv\left[m_{1}:\right.$ $\varepsilon]|\ldots|\left[m_{p}: \varepsilon\right]\left|\left[n_{1}: \varepsilon\right]\right| \ldots\left|\left[n_{k}: \varepsilon\right]\right| \operatorname{cl}(P) \equiv \operatorname{cl}_{\sigma}\left(\left[n_{1}: \varepsilon\right]|\ldots|\left[n_{k}: \varepsilon\right] \mid P\right)$.

Lemma 47 If $n \notin f n(\sigma), c l_{\sigma}((\nu n) P) \equiv \begin{cases}(\nu n) c l_{\sigma}(P) & \text { if } n \in f n(P) \\ (\nu n)\left([n: \varepsilon] \mid c l_{\sigma}(P)\right) & \text { if } n \notin f n(P)\end{cases}$

\section{Proof:}

- If $P \Downarrow_{1} n$, then $n \in f n(P)$. In this case, $\operatorname{pr}(P)=\{n\} \cup \operatorname{pr}((\nu n) P)$ and $\operatorname{cl}((\nu n) P)=$ $(\nu n) c l(P)$. Then, $(f n((\nu n) P) \cup f n(\sigma)) \backslash p r((\nu n) P)=(f n(P) \backslash\{n\} \cup f n(\sigma)) \backslash p r((\nu n) P)=$ $((f n(P) \cup f n(\sigma)) \backslash\{n\}) \backslash p r((\nu n) P)$ since $n \notin f n(\sigma)$. This is equal to $(f n(P) \cup f n(\sigma)) \backslash p r(P)$. Finally, $c_{\sigma}((\nu n) P) \equiv\left[n_{1}: \varepsilon\right]|\ldots|\left[n_{k}: \varepsilon\right] \mid(\nu n) c l(P) \equiv(\nu n)\left(\left[n_{1}: \varepsilon\right]|\ldots|\left[n_{k}:\right.\right.$ $\varepsilon] \mid c l(P)) \equiv(\nu n) c l_{\sigma}(P)$, using $\left(\pi_{e s c}\right.$ Struct Res Par) (we can suppose $\left.n_{i} \neq n\right)$.

- If $P \Downarrow_{1} n$, we have $\operatorname{pr}(P)=\operatorname{pr}((\nu n) P)$ and $\operatorname{cl}((\nu n) P)=(\nu n)([n: \varepsilon] \mid \operatorname{cl}(P))$. There are two cases to consider:

- If $n \notin f n(P), f n((\nu n) P)=f n(P)$. Then, $(f n((\nu n) P) \cup f n(\sigma)) \backslash p r((\nu n) P)=$ $(f n(P) \cup f n(\sigma)) \backslash p r(P)$. Finally, $c l_{\sigma}((\nu n) P) \equiv\left[n_{1}: \varepsilon\right]|\ldots|\left[n_{k}: \varepsilon\right] \mid(\nu n)([n:$ $\varepsilon] \mid c l(P)) \equiv(\nu n)\left([n: \varepsilon]\left|\left[n_{1}: \varepsilon\right]\right| \ldots\left|\left[n_{k}: \varepsilon\right]\right| c l(P)\right) \equiv(\nu n)\left([n: \varepsilon] \mid c l_{\sigma}(P)\right)$.

- If $n \in f n(P), f n((\nu n) P) \cup\{n\}=f n(P)$. Then, $(f n(P) \cup f n(\sigma)) \backslash p r(P)=(f n((\nu n) P) \cup$ $\{n\} \cup f n(\sigma)) \backslash p r((\nu n) P)=(f n((\nu n) P) \cup f n(\sigma)) \backslash p r((\nu n) P) \cup\{n\}$ (since $n \notin$ $\operatorname{pr}((\nu n) P))$. Finally, $c l_{\sigma}((\nu n) P) \equiv\left[n_{1}: \varepsilon\right]|\ldots|\left[n_{k}: \varepsilon\right] \mid(\nu n)([n: \varepsilon] \mid c l(P)) \equiv$ $(\nu n)\left(\left[n_{1}: \varepsilon\right]|\ldots|\left[n_{k}: \varepsilon\right]|[n: \varepsilon]| c l(P)\right) \equiv(\nu n) c l_{\sigma}(P)$. 


\section{A.4 Managing Empty Channels}

In this Section, we adress our second problem: in $\pi_{e s c}$-calculus, empty channels can be "erased" by structural congruence rewriting. To avoid this effect, we introduce an ordering relation on terms, modulo $\equiv$, written $\preccurlyeq$. Intuitively, $P \preccurlyeq P^{\prime}$ means that $P^{\prime}$ is similar to $P$, but contains some extra empty channels. As an example, the main axiom is $\mathbf{0} \preccurlyeq[n: \varepsilon]$.

More formally, $\preccurlyeq$ is the least relation on terms satisfying the following rules:

$$
\begin{array}{ll}
P \equiv Q \Rightarrow P \preccurlyeq Q & \left(\pi_{e s c}\right. \text { Empty Struct) } \\
P \preccurlyeq Q \preccurlyeq R \Rightarrow P \preccurlyeq R & \left(\pi_{e s c} \text { Empty Trans }\right) \\
\mathbf{0} \preccurlyeq[n: \varepsilon] & \left(\pi_{e s c} \text { Empty Axiom }\right) \\
P \preccurlyeq P^{\prime} \Rightarrow(\nu n) P \preccurlyeq(\nu n) P^{\prime} & \left(\pi_{e s c} \text { Empty Res }\right) \\
\mathbf{0} \preccurlyeq \mathbf{0} & \left(\pi_{e s c}\right. \text { Empty Zero) } \\
P \preccurlyeq P^{\prime} \text { and } Q \preccurlyeq Q^{\prime} \Rightarrow P\left|Q \preccurlyeq P^{\prime}\right| Q^{\prime} & \left(\pi_{e s c}\right. \text { Empty Par) } \\
P \preccurlyeq P^{\prime} \Rightarrow ! P \preccurlyeq ! P^{\prime} & \left(\pi_{e s c} \text { Empty Repl }\right) \\
P \preccurlyeq P^{\prime} \Rightarrow \bar{M}\left\langle M^{\prime}\right\rangle \cdot P \preccurlyeq \bar{M}\left\langle M^{\prime}\right\rangle \cdot P^{\prime} & \left(\pi_{e s c}\right. \text { Empty Output) } \\
P \preccurlyeq P^{\prime} \Rightarrow M(x) \cdot P \preccurlyeq M(x) \cdot P^{\prime} & \left(\pi_{e s c} \text { Empty Input }\right) \\
S \preccurlyeq S^{\prime} \Rightarrow[n: S] \preccurlyeq\left[n: S^{\prime}\right] & \left(\pi_{e s c}\right. \text { Empty Channel) } \\
P \preccurlyeq P^{\prime} \Rightarrow(\nu x: M) P \preccurlyeq(\nu x: M) P^{\prime} & \left(\pi_{e s c} \text { Empty Var }\right) \\
\varepsilon \preccurlyeq \varepsilon & \left(\pi_{e s c}\right. \text { Empty Empty) } \\
S \preccurlyeq S_{1} \text { and } S^{\prime} \preccurlyeq S_{1}^{\prime} \Rightarrow S\left|S^{\prime} \preccurlyeq S_{1}\right| S_{1}^{\prime} & \left(\pi_{e s c} \text { Empty Abs }\right) \\
P \preccurlyeq P^{\prime} \Rightarrow\langle M\rangle \cdot P \preccurlyeq\langle M\rangle \cdot P^{\prime} & \left(\pi_{e s c}\right. \text { Empty Out Abs) } \\
P \preccurlyeq P^{\prime} \Rightarrow(x) \cdot P \preccurlyeq(x) \cdot P^{\prime} & \left(\pi_{e s c}\right. \text { Empty Inp Abs) }
\end{array}
$$

First of all, in order to understand why we need such a technical tool, let us explain why we could not have imagined to add the rule $\mathbf{0} \equiv[n: \varepsilon]$ to the definition of structural congruence. In that case, we would have:

$$
\begin{array}{lll} 
& \bar{n}\langle M\rangle \cdot P \mid n(x) \cdot Q \\
\equiv & {[n: \varepsilon]|[n: \varepsilon]| \bar{n}\langle M\rangle \cdot P \mid n(x) \cdot Q} \\
\longmapsto \longmapsto & {[n:\langle M\rangle \cdot P] \mid[n:(x) \cdot Q]}
\end{array}
$$

and no further communication could occur. This problem is very similar to the one that motivated the introduction of the presentation predicates $P \Downarrow_{1} n$ and $P \Downarrow_{2} n \ldots$

Lemma $48 \preccurlyeq$ is an ordering relation, modulo $\equiv$.

Proof: Check that:

- $\preccurlyeq$ is reflexive: $P \preccurlyeq P$ since $P \equiv P$.

- $\preccurlyeq$ is transitive: by definition.

- $\preccurlyeq$ is asymmetric modulo $\equiv$, i.e. $P \preccurlyeq P^{\prime}$ and $P^{\prime} \preccurlyeq P$ implies $P \equiv P^{\prime}$ : by induction on the derivation of $P \preccurlyeq P^{\prime}$. 
The next few lemmas show how the relation $\preccurlyeq$ relates to free names and variables, channel presentation, validity and finally channel closure.

Lemma 49 If $P \preccurlyeq P^{\prime}$, then $f n(P) \subseteq f n\left(P^{\prime}\right)$ and $f v(P)=f v\left(P^{\prime}\right)$.

Proof: By induction on the derivation of $P \preccurlyeq P^{\prime}$.

Lemma 50 If $P \preccurlyeq P^{\prime}$ and $P \Downarrow_{i} n$, then $P^{\prime} \Downarrow_{i} n$.

Proof: By induction on the derivation of $P \preccurlyeq P^{\prime}$, first for $i=1$, then for $i=2$, using Lemma 32 .

Corollary 51 If $P \preccurlyeq P^{\prime}$, then $\operatorname{pr}(P) \subseteq \operatorname{pr}\left(P^{\prime}\right)$.

Proof: Using Lemma 50.

Lemma 52 If $P \preccurlyeq P^{\prime}$ and $\vdash P^{\prime}:$ Valid, then $\vdash P$ : Valid.

Proof: By induction on the derivation of $P \preccurlyeq P^{\prime}$, using Lemma 50 .

Lemma 53 If $P \preccurlyeq P^{\prime}$ and $\vdash P^{\prime}:$ Valid, then $\operatorname{cl}\left(P^{\prime}\right) \equiv\left[n_{1}: \varepsilon\right]|\ldots|\left[n_{k}: \varepsilon\right] \mid \operatorname{cl}(P)$ with $\left\{n_{1}, \ldots, n_{k}\right\}=\operatorname{pr}\left(P^{\prime}\right) \backslash \operatorname{pr}(P)$.

Proof: By induction on the derivation of $P \preccurlyeq P^{\prime}$, using Corollary 51,

( $\pi_{e s c}$ Empty Struct) $\quad$ Suppose $P \equiv P^{\prime}$. Then, $c l(P) \equiv c l\left(P^{\prime}\right)$ by Lemma 32, and $\operatorname{pr}(P)=\operatorname{pr}\left(P^{\prime}\right)$ by Corollary 33 .

( $\pi_{e s c}$ Empty Trans) Suppose $P \preccurlyeq Q \preccurlyeq R$ and $\vdash R$ : Valid. By Lemma $52, \vdash Q:$ Valid. By induction hypothesis,

$$
\begin{aligned}
c l(R) & \equiv\left[n_{1}: \varepsilon\right]|\ldots|\left[n_{k}: \varepsilon\right] \mid \operatorname{cl}(Q) \\
& \equiv\left[n_{1}: \varepsilon\right]|\ldots|\left[n_{k}: \varepsilon\right]\left|\left[m_{1}: \varepsilon\right]\right| \ldots\left|\left[m_{p}: \varepsilon\right]\right| \operatorname{cl}(P)
\end{aligned}
$$

with $\left\{n_{1}, \ldots, n_{k}\right\}=\operatorname{pr}(R) \backslash \operatorname{pr}(Q)$ and $\left\{m_{1}, \ldots, m_{p}\right\}=\operatorname{pr}(Q) \backslash \operatorname{pr}(P)$. It remains to check that $\operatorname{pr}(R) \backslash \operatorname{pr}(P)=\left\{n_{1}, \ldots, n_{k}, m_{1}, \ldots, m_{p}\right\}$, which is easy by Corollary 51 .

( $\pi_{e s c}$ Empty Axiom) We have $c l([n: \varepsilon])=[n: \varepsilon] \equiv[n: \varepsilon]|\mathbf{0}=[n: \varepsilon]| \operatorname{cl}(\mathbf{0})$ and $\operatorname{pr}([n:$ $\varepsilon]) \backslash p r(\mathbf{0})=\{n\}$.

( $\pi_{e s c}$ Empty Res) Suppose that $(\nu n) P \preccurlyeq(\nu n) P^{\prime}$ was derived from $P \preccurlyeq P^{\prime}$. Since $\vdash(\nu n) P^{\prime}$ : Valid, $\vdash P^{\prime}:$ Valid. By induction hypothesis, $\operatorname{cl}\left(P^{\prime}\right) \equiv\left[n_{1}: \varepsilon\right]|\ldots|\left[n_{k}: \varepsilon\right] \mid \operatorname{cl}(P)$ with $\left\{n_{1}, \ldots, n_{k}\right\}=\operatorname{pr}\left(P^{\prime}\right) \backslash \operatorname{pr}(P)$. There are three cases to consider:

- If $P \Downarrow_{1} n$, then $P^{\prime} \Downarrow_{1} n$ by Lemma 50 , and we have $n \neq n_{i}$. Moreover, $\operatorname{pr}\left((\nu n) P^{\prime}\right) \backslash$ $\operatorname{pr}((\nu n) P)=\left\{n_{1}, \ldots, n_{k}\right\}$, and

$$
\begin{aligned}
\operatorname{cl}\left((\nu n) P^{\prime}\right) & =(\nu n) \operatorname{cl}\left(P^{\prime}\right) \\
& \equiv(\nu n)\left(\left[n_{1}: \varepsilon\right]|\ldots|\left[n_{k}: \varepsilon\right] \mid \operatorname{cl}(P)\right) \\
& \equiv\left[n_{1}: \varepsilon\right]|\ldots|\left[n_{k}: \varepsilon\right] \mid(\nu n) \operatorname{cl}(P) \\
& =\left[n_{1}: \varepsilon\right]|\ldots|\left[n_{k}: \varepsilon\right] \mid \operatorname{cl}((\nu n) P)
\end{aligned}
$$

$\mathrm{RR} \mathrm{n}^{\circ} 4350$ 
- If $P \Downarrow_{1} n$ and $P^{\prime} \Downarrow_{1} n, n$ is one of the $n_{i}$. We may assume $n=n_{1}$ for example. Then, we have $\operatorname{pr}\left((\nu n) P^{\prime}\right) \backslash p r((\nu n) P)=\left\{n_{2}, \ldots, n_{k}\right\}$, and

$$
\begin{aligned}
\operatorname{cl}\left((\nu n) P^{\prime}\right) & =(\nu n) \operatorname{cl}\left(P^{\prime}\right) \\
& \equiv(\nu n)\left([n: \varepsilon]\left|\left[n_{2}: \varepsilon\right]\right| \ldots\left|\left[n_{k}: \varepsilon\right]\right| \operatorname{cl}(P)\right) \\
& \equiv\left[n_{2}: \varepsilon\right]|\ldots|\left[n_{k}: \varepsilon\right] \mid(\nu n)([n: \varepsilon] \mid \operatorname{cl}(P)) \\
& =\left[n_{2}: \varepsilon\right]|\ldots|\left[n_{k}: \varepsilon\right] \mid \operatorname{cl}((\nu n) P)
\end{aligned}
$$

- If $P \Downarrow_{1} n$ and $P^{\prime} \Downarrow_{1} n$, we have $n \neq n_{i}$ and $\operatorname{pr}\left((\nu n) P^{\prime}\right) \backslash p r((\nu n) P)=\left\{n_{1}, \ldots, n_{k}\right\}$. Moreover,

$$
\begin{aligned}
\operatorname{cl}\left((\nu n) P^{\prime}\right) & =(\nu n)\left([n: \varepsilon] \mid \operatorname{cl}\left(P^{\prime}\right)\right) \\
& \equiv(\nu n)\left([n: \varepsilon]\left|\left[n_{1}: \varepsilon\right]\right| \ldots\left|\left[n_{k}: \varepsilon\right]\right| \operatorname{cl}(P)\right) \\
& \equiv\left[n_{1}: \varepsilon\right]|\ldots|\left[n_{k}: \varepsilon\right] \mid(\nu n)([n: \varepsilon] \mid \operatorname{cl}(P)) \\
& =\left[n_{1}: \varepsilon\right]|\ldots|\left[n_{k}: \varepsilon\right] \mid \operatorname{cl}((\nu n) P)
\end{aligned}
$$

( $\pi_{e s c}$ Empty Par) Suppose that $P\left|Q \preccurlyeq P^{\prime}\right| Q^{\prime}$ was derived from $P \preccurlyeq P^{\prime}$ and $Q \preccurlyeq Q^{\prime}$. Since $\vdash P^{\prime} \mid Q^{\prime}$ : Valid, we have $\vdash P^{\prime}:$ Valid and $\vdash Q^{\prime}:$ Valid. By induction hypothesis, $c l\left(P^{\prime}\right) \equiv\left[n_{1}: \varepsilon\right]|\ldots|\left[n_{k}: \varepsilon\right] \mid \operatorname{cl}(P)$ with $\left\{n_{1}, \ldots, n_{k}\right\}=\operatorname{pr}\left(P^{\prime}\right) \backslash \operatorname{pr}(P)$, and $c l\left(Q^{\prime}\right) \equiv\left[m_{1}\right.$ : $\varepsilon]|\ldots|\left[m_{p}: \varepsilon\right] \mid \operatorname{cl}(Q)$ with $\left\{m_{1}, \ldots, m_{p}\right\}=\operatorname{pr}\left(Q^{\prime}\right) \backslash \operatorname{pr}(Q)$. Since $\vdash P^{\prime} \mid Q^{\prime}:$ Valid, we have $n_{i} \neq m_{j}$ (otherwise $P^{\prime} \mid Q^{\prime} \Downarrow_{2} n_{i}$ ). Moreover, $\operatorname{pr}\left(P^{\prime}\right) \cap \operatorname{pr}(Q) \subseteq \operatorname{pr}\left(P^{\prime}\right) \cap \operatorname{pr}\left(Q^{\prime}\right)=\emptyset$ and $\operatorname{pr}\left(Q^{\prime}\right) \cap \operatorname{pr}(P)=\emptyset$. Consequently, $\operatorname{pr}\left(P^{\prime} \mid Q^{\prime}\right) \backslash \operatorname{pr}(P \mid Q)=\operatorname{pr}\left(P^{\prime}\right) \backslash p r(P) \cup \operatorname{pr}\left(Q^{\prime}\right) \backslash p r(Q)=$ $\left\{n_{1}, \ldots, n_{k}, m_{1}, \ldots, m_{p}\right\}$. Finally, $\operatorname{cl}\left(P^{\prime} \mid Q^{\prime}\right)=\operatorname{cl}\left(P^{\prime}\right)\left|\operatorname{cl}\left(Q^{\prime}\right) \equiv\left[n_{1}: \varepsilon\right]\right| \ldots\left|\left[n_{k}: \varepsilon\right]\right|\left[m_{1}:\right.$ $\varepsilon]|\ldots|\left[m_{p}: \varepsilon\right] \mid \operatorname{cl}(P \mid Q)$.

( $\pi_{\text {esc }}$ Empty Repl) Suppose that $! P \preccurlyeq ! P^{\prime}$ was derived from $P \preccurlyeq P^{\prime}$. Since $\vdash ! P^{\prime}:$ Valid, we have $\operatorname{pr}\left(P^{\prime}\right)=\emptyset$. Then, by induction hypothesis, $\operatorname{cl}\left(P^{\prime}\right) \equiv \operatorname{cl}(P)$. And, since $\operatorname{pr}\left(! P^{\prime}\right)=\emptyset$, we have $c l\left(! P^{\prime}\right) \equiv \operatorname{cl}(! P)$.

The other cases are similar or trivial.

Finally, the following result show that if we translate a process $P$ in $\pi$-calculus and rewrite it by structural congruence (thus possibly erasing some empty channels), we get in fact the direct translation of a process $\preccurlyeq P$.

Lemma 54 If $\llbracket P \rrbracket \equiv Q$ (or $Q \equiv \llbracket P \rrbracket$ ), then there exists $P^{\prime}$ such that $Q=\llbracket P^{\prime} \rrbracket$ and $P^{\prime} \preccurlyeq P$ (and the same for $S$ instead of $P$ ).

Proof: By induction on the derivation of $\llbracket P \rrbracket \equiv Q$, with a special treatment for $P=(\nu x: M) P^{\prime}$ : since this part of the reasoning is common to all cases, we include it in the induction and we consider that $P$ is not of this form in all other cases.

$(\nu \mathbf{x}: \mathbf{M}) \mathbf{P}$ We have $\llbracket(\nu x: M) P \rrbracket=\llbracket P \rrbracket\left\{{ }^{M} / x\right\} \equiv Q$. By Lemma 29 , there is $Q^{\prime}$ such that $\llbracket P \rrbracket \equiv Q^{\prime}$ and $Q=Q^{\prime}\left\{{ }^{M} / x\right\}$. By induction hypothesis, there is $P^{\prime}$ such that $Q^{\prime}=\llbracket P^{\prime} \rrbracket$ and $P^{\prime} \preccurlyeq P$. Then, $Q=\llbracket P^{\prime} \rrbracket\left\{{ }^{M} / x\right\}=\llbracket(\nu x: M) P^{\prime} \rrbracket$ and we can derive $(\nu x: M) P^{\prime} \preccurlyeq(\nu x: M) P$.

( $\pi$ Struct Refl) Trivial, because $\preccurlyeq$ is reflexive.

( $\pi$ Struct Symm) Directly by induction.

INRIA 
( $\pi$ Struct Trans) Trivial, because $\preccurlyeq$ is transitive.

( $\pi$ Struct Res) Suppose $\llbracket P \rrbracket=(\nu n) P_{1} \equiv(\nu n) Q_{1}=Q$ was derived from $P_{1} \equiv Q_{1}$. Necessarily, $P=(\nu n) P_{2}$ with $P_{1}=\llbracket P_{2} \rrbracket$. By induction hypothesis, there is $P_{2}^{\prime}$ such that $Q_{1}=\llbracket P_{2}^{\prime} \rrbracket$ and $P_{2}^{\prime} \preccurlyeq P_{2}$. Let $P^{\prime}=(\nu n) P_{2}^{\prime}$. We have $Q=(\nu n) \llbracket P_{2}^{\prime} \rrbracket=\llbracket P^{\prime} \rrbracket$ and $P^{\prime} \preccurlyeq(\nu n) P_{2}=P$.

( $\pi$ Struct Par) Suppose that $\llbracket P \rrbracket=P_{0}|R \equiv Q| R$ was derived from $P_{0} \equiv Q$. By Lemma 43, there are two cases to consider:

- $P=P_{1} \mid P_{2}$ with $\llbracket P_{1} \rrbracket=P_{0}$ and $\llbracket P_{2} \rrbracket=R$. We have $\llbracket P_{1} \rrbracket \equiv Q$. By induction hypothesis, there is $P_{1}^{\prime}$ such that $Q=\llbracket P_{1}^{\prime} \rrbracket$ and $P_{1}^{\prime} \preccurlyeq P_{1}$. Then, $Q\left|R=\llbracket P_{1}^{\prime}\right| P_{2} \rrbracket$ and $P_{1}^{\prime}\left|P_{2} \preccurlyeq P_{1}\right| P_{2}=P$.

- $P=\left[n: S_{1} \mid S_{2}\right]$ with $\llbracket S_{1} \rrbracket_{n}=P_{0}$ and $\llbracket S_{2} \rrbracket_{n}=R$. We have $\llbracket S_{1} \rrbracket_{n} \equiv Q$. By induction hypothesis, there is $S_{1}^{\prime}$ such that $Q=\llbracket S_{1}^{\prime} \rrbracket_{n}$ and $S_{1}^{\prime} \preccurlyeq S_{1}$. Then, $Q\left|R=\llbracket S_{1}^{\prime}\right| S_{2} \rrbracket_{n}=$ $\llbracket\left[n: S_{1}^{\prime} \mid S_{2}\right] \rrbracket$ and $\left[n: S_{1}^{\prime} \mid S_{2}\right] \preccurlyeq\left[n: S_{1} \mid S_{2}\right]=P$.

( $\pi$ Struct Par Zero) Suppose $\llbracket P \rrbracket=Q \mid \mathbf{0} \equiv Q$. By Lemma 43, there are two cases to consider:

- $P=\left[n: S \mid S^{\prime}\right]$ with $\llbracket S \rrbracket_{n}=Q$ and $\llbracket S^{\prime} \rrbracket_{n}=\mathbf{0}$. Necessarily, $S^{\prime}=\varepsilon$. We have $P=[n: S \mid \varepsilon] \equiv[n: S]$. Finally, $Q=\llbracket[n: S] \rrbracket$ and $[n: S] \preccurlyeq P$.

- $P=P_{1} \mid P_{2}$ with $\llbracket P_{1} \rrbracket=Q$ and $\llbracket P_{2} \rrbracket=\mathbf{0}$. There are two possibilities. If $P_{2}=\mathbf{0}$, then $P=P_{1} \mid \mathbf{0} \equiv P_{1}$, so that $P_{1} \preccurlyeq P$. Otherwise, $P_{2}=[n: \varepsilon]$, and then $P_{1} \equiv P_{1} \mid \mathbf{0} \preccurlyeq$ $P_{1} \mid[n: \varepsilon]=P$.

The other cases are similar. 


\section{A.5 Channels and Parallel Composition}

In this Section, we adress the third problem: to complete an inductive proof, we need to show how communications involving an empty channel added by channel closure can be reported to (possibly non-empty) channels provided by a process in parallel.

As a first step, we define a syntactic operator that finds the channels $n_{i}$ in a process and adds some concretions and abstractions $S_{i}$ in it.

Definition $55 P\left\{n_{i} \leftarrow S_{i}\right\}$ is defined by $\left[n_{j}: S^{\prime}\right]\left\{n_{i} \leftarrow S_{i}\right\}=\left[n_{j}: S^{\prime}\left\{n_{i}+S_{i}\right\} \mid S_{j}\right]$ and is an homomorphism for all other constructs.

Next, we show that this operator is preserved by structural congruence and reduction in $\pi_{\text {esc }}$.

\section{Lemma 56}

- If $P \equiv Q$, then $P\left\{n_{i} \leftrightarrow S_{i}\right\} \equiv Q\left\{n_{i} \leftarrow S_{i}\right\}$.

- If $f v\left(S_{i}\right) \subseteq \operatorname{dom}(\sigma)$ and $\sigma: P \longmapsto P^{\prime}$, then $\sigma: P\left\{n_{i} \nleftarrow S_{i}\right\} \longmapsto P^{\prime}\left\{n_{i} \nleftarrow S_{i}\right\}$.

\section{Proof:}

- By induction on the derivation of $P \equiv Q$.

- By induction on the derivation of $\sigma: P \longmapsto P^{\prime}$.

This lemma shows that translating a $\pi_{e s c}$-process back into $\pi$-calculus after some channel extensions is equivalent to translating the original process and the extensions separately, as if these were simple input/output instructions. Thus, we "forget" the first step of communication (the entering of a channel).

Lemma 57 If $\vdash P:$ Valid, then $\llbracket P\left\{n_{i}+S_{i}\right\} \rrbracket \equiv \llbracket P \rrbracket\left|\llbracket S_{i_{1}} \rrbracket_{n_{i_{1}}}\right| \ldots \mid \llbracket S_{i_{k}} \rrbracket_{n_{i_{k}}}$ where $\left\{i_{1}, \ldots, i_{k}\right\}=\left\{i / P \Downarrow_{1} n_{i}\right\}$.

Proof: By induction on the structure of $P$.

- $\llbracket((\nu n) P)\left\{n_{i}+S_{i}\right\} \rrbracket=(\nu n) \llbracket P\left\{n_{i}+S_{i}\right\} \rrbracket$ (we can always suppose $n \notin f n\left(\left\{n_{i} \leftarrow S_{i}\right\}\right)$. Since $\vdash(\nu n) P:$ Valid implies $\vdash P:$ Valid, we have $\llbracket P\left\{n_{i}+S_{i}\right\} \rrbracket \equiv \llbracket P \rrbracket\left|\llbracket S_{i_{1}} \rrbracket \rrbracket_{n_{i_{1}}}\right| \ldots \mid \llbracket S_{i_{k}} \rrbracket_{n_{i_{k}}}$ with $\left\{i_{1}, \ldots, i_{k}\right\}=\left\{i / P \Downarrow_{1} n_{i}\right\}$ by induction hypothesis. Since $n \neq n_{i}$, we have $\left\{i /(\nu n) P \Downarrow_{1}\right.$ $\left.n_{i}\right\}=\left\{i / P \Downarrow_{1} n_{i}\right\}$. Finally, $\llbracket((\nu n) P)\left\{n_{i}+S_{i}\right\} \rrbracket \equiv(\nu n)\left(\llbracket P \rrbracket\left|\llbracket S_{i_{1}} \rrbracket_{n_{i_{1}}}\right| \ldots \mid \llbracket S_{i_{k}} \rrbracket_{n_{i_{k}}}\right) \equiv$ $\llbracket(\nu n) P \rrbracket\left|\llbracket S_{i_{1}} \rrbracket_{n_{i_{1}}}\right| \ldots \mid \llbracket S_{i_{k}} \rrbracket_{n_{i_{k}}}$.

- $\llbracket \mathbf{0}\left\{n_{i}+S_{i}\right\} \rrbracket=\llbracket \mathbf{0} \rrbracket$ and $\left\{i / \mathbf{0} \Downarrow_{1} n_{i}\right\}=\emptyset$ trivially.

- $\llbracket(P \mid Q)\left\{n_{i} \longleftrightarrow S_{i}\right\} \rrbracket=\llbracket P\left\{n_{i} \longleftarrow S_{i}\right\} \rrbracket \mid \llbracket Q\left\{n_{i} \longleftrightarrow S_{i}\right\} \rrbracket$. Since $\vdash P \mid Q$ : Valid, $\vdash$ $P:$ Valid and $\vdash Q$ : Valid. Moreover, we cannot have $P \Downarrow_{1} n_{i}$ and $Q \Downarrow_{1} n_{i}$ at the same time, otherwise we would have $P \mid Q \Downarrow_{2} n_{i}$. Thus, $\left\{i / P \mid Q \Downarrow_{1} n_{i}\right\}=\left\{i / P \Downarrow_{1}\right.$ $\left.n_{i}\right\} \cup\left\{i / Q \Downarrow_{1} n_{i}\right\}$ with a disjunctive union. By induction hypothesis, $\llbracket P\left\{n_{i} \longleftrightarrow S_{i}\right\} \rrbracket \equiv$ $\llbracket P \rrbracket\left|\llbracket S_{i_{1}} \rrbracket_{n_{i_{1}}}\right| \ldots \mid \llbracket S_{i_{k}} \rrbracket_{n_{i_{k}}}$ where $\left\{i_{1}, \ldots, i_{k}\right\}=\left\{i / P \Downarrow_{1} n_{i}\right\}$, and $\llbracket Q\left\{n_{i}+S_{i}\right\} \rrbracket \equiv$ $\llbracket Q \rrbracket\left|\llbracket S_{j_{1}} \rrbracket_{n_{j_{1}}}\right| \ldots \mid \llbracket S_{j_{p}} \rrbracket_{n_{j_{p}}}$ where $\left\{j_{1}, \ldots, j_{p}\right\}=\left\{i / Q \Downarrow_{1} n_{i}\right\}$. Then, $\llbracket(P \mid Q)\left\{n_{i}+S_{i}\right\} \rrbracket \equiv$ $\llbracket P|Q \rrbracket| \llbracket S_{i_{1}} \rrbracket_{n_{i_{1}}}|\ldots| \llbracket \llbracket S_{i_{k}} \rrbracket_{n_{i_{k}}}\left|\llbracket S_{j_{1}} \rrbracket_{n_{j_{1}}}\right| \ldots \mid \llbracket S_{j_{p}} \rrbracket_{n_{j_{p}}}$ with $\left\{i_{1}, \ldots, i_{k}, j_{1}, \ldots, j_{p}\right\}=$ $\left\{i / P \mid Q \Downarrow_{1} n_{i}\right\}$. 
- Since $\vdash ! P$ : Valid, $P \Downarrow_{1} n$ for all $n \in$ Name. Moreover, $\vdash P:$ Valid and $\llbracket P\left\{n_{i} \leftarrow S_{i}\right\} \rrbracket=\llbracket P \rrbracket$ by induction hypothesis. Then, $\llbracket(! P)\left\{n_{i} \leftrightarrow S_{i}\right\} \rrbracket=! \llbracket P\left\{n_{i} \leftarrow S_{i}\right\} \rrbracket=! \llbracket P \rrbracket=\llbracket ! P \rrbracket$, and $\left\{i / ! P \Downarrow_{1} n_{i}\right\}=\left\{i / P \Downarrow_{1} n_{i}\right\}=\emptyset$.

- $\llbracket\left[n_{j}: S\right]\left\{n_{i}+S_{i}\right\} \rrbracket=\llbracket\left[n_{j}: S\left\{n_{i}+S_{i}\right\} \mid S_{j}\right] \rrbracket=\llbracket S\left\{n_{i}+S_{i}\right\} \rrbracket_{n_{j}} \mid \llbracket S_{j} \rrbracket_{n_{j}}$. Since $\vdash\left[n_{j}: S\right]:$ Valid, $\vdash S:$ Valid. By induction hypothesis, $\llbracket S\left\{n_{i}+S_{i}\right\} \rrbracket_{n_{j}} \equiv \llbracket S \rrbracket_{n_{j}}\left|\llbracket S_{i_{1}} \rrbracket_{n_{i_{1}}}\right| \ldots \mid \llbracket S_{i_{k}} \rrbracket_{n_{i_{k}}}$ where $\left\{i_{1}, \ldots, i_{k}\right\}=\left\{i / S \Downarrow_{1} n_{i}\right\}$. Then, $\llbracket\left[n_{j}: S\right]\left\{n_{i}+S_{i}\right\} \rrbracket \equiv \llbracket\left[n_{j}: S\right] \rrbracket\left|\llbracket S_{i_{1}} \rrbracket_{n_{i_{1}}}\right| \ldots\left|\llbracket S_{i_{k}} \rrbracket_{n_{i_{k}}}\right| \llbracket S_{j} \rrbracket_{n_{j}}$. And $\left\{i /\left[n_{j}: S\right] \Downarrow_{1} n_{i}\right\}=\{j\} \cup\left\{i / S \Downarrow_{1} n_{i}\right\}$ with a disjunctive union (otherwise $\left[n_{j}: S\right] \Downarrow_{2} n_{j}$ which is impossible since $\vdash\left[n_{j}: S\right]:$ Valid $)$.

The other cases are very similar.

This lemma shows that we can increase the contents of channels placed in parallel without affecting the possible reductions.

Lemma 58 If $\vdash P:$ Valid, $P \Downarrow_{1} n_{i}, f v\left(S_{i}\right) \subseteq \operatorname{dom}(\sigma)$ and $\sigma:\left[n_{1}: \varepsilon\right]|\ldots|\left[n_{k}:\right.$ $\varepsilon] \mid P \longmapsto^{*} P^{\prime}$, then $\sigma:\left[n_{1}: S_{1}\right]|\ldots|\left[n_{k}: S_{k}\right] \mid P \longmapsto^{*} P^{\prime \prime}$ with the condition $\llbracket P^{\prime \prime} \rrbracket \equiv \llbracket P^{\prime} \rrbracket\left|\llbracket S_{1} \rrbracket_{n_{1}}\right| \ldots \mid \llbracket S_{k} \rrbracket_{n_{k}}$.

Proof: Let $Q=\left[n_{1}: \varepsilon\right]|\ldots|\left[n_{k}: \varepsilon\right] \mid P$. By Lemma 56, $\sigma: Q\left\{n_{i}+S_{i}\right\} \longmapsto{ }^{*} P^{\prime}\left\{n_{i} \leftarrow S_{i}\right\}$. Since $P \Downarrow_{1} n_{i}, P\left\{n_{i}+S_{i}\right\}=P$ and thus $Q\left\{n_{i}+S_{i}\right\}=\left[n_{1}: S_{1}\right]|\ldots|\left[n_{k}: S_{k}\right] \mid P$. Moreover, since $P \Downarrow_{1} n_{i}, Q \Downarrow_{1} n_{i}$ but $Q \Downarrow_{2} n_{i}$. It follows easily that $\vdash Q:$ Valid. By Proposition 6 , $\vdash P^{\prime}$ : Valid. Using Lemma 57, we get $\llbracket P^{\prime}\left\{n_{i}+S_{i}\right\} \rrbracket=\llbracket P^{\prime} \rrbracket\left|\llbracket S_{1} \rrbracket n_{1}\right| \ldots \mid \llbracket S_{k} \rrbracket_{n_{k}}\left(P^{\prime} \Downarrow_{1} n_{i}\right.$ for all $i$ because of Lemma 34 and $Q \Downarrow_{1} n_{i}$ ).

This lemma generalizes the previous one by showing that we can use the channels of an other process in parallel if they are available, instead of empty channels.

Lemma 59 If $\vdash P:$ Valid, $\vdash Q:$ Valid, $P \Downarrow_{1} n_{i}, Q \Downarrow_{1} n_{i}, f v(Q) \subseteq \operatorname{dom}(\sigma)$ and $\sigma:\left[n_{1}:\right.$ $\varepsilon]|\ldots|\left[n_{k}: \varepsilon\right] \mid P \longmapsto{ }^{*} P^{\prime}$, then $\sigma: P \mid Q \longmapsto{ }^{*} P^{\prime \prime}$ with $\llbracket P^{\prime \prime} \rrbracket \equiv \llbracket P^{\prime} \mid Q \rrbracket$.

Proof: From Corollary 5, we get $Q \equiv\left(\nu m_{1}\right) \ldots\left(\nu m_{p}\right)\left(\nu x_{1}: M_{1}\right) \ldots\left(\nu x_{p^{\prime}}: M_{p^{\prime}}\right)\left(\left[n_{1}:\right.\right.$ $\left.\left.S_{1}\right]|\ldots|\left[n_{k}: S_{k}\right] \mid Q^{\prime}\right)$ with $n_{i} \neq m_{j}$. We also suppose $n_{i} \notin f n(P) \cup f n\left(\llbracket P^{\prime} \rrbracket\right), x_{i} \notin f v(P) \cup$ $f v\left(\llbracket P^{\prime} \rrbracket\right) \cup \operatorname{dom}(\sigma)$ and $x_{i} \neq x_{j}$ for $i \neq j$. Then, $P \mid Q \equiv\left(\nu m_{1}\right) \ldots\left(\nu m_{p}\right)\left(\nu x_{1}: M_{1}\right) \ldots\left(\nu x_{p^{\prime}}:\right.$ $\left.M_{p^{\prime}}\right)\left(\left[n_{1}: S_{1}\right]|\ldots|\left[n_{k}: S_{k}\right]|P| Q^{\prime}\right)$. By Lemma 39, we have $\left\{{ }^{M_{p^{\prime}}} /{ }_{x_{p^{\prime}}}\right\} \uplus \ldots \uplus\left\{{ }^{M_{1}} / x_{1}\right\} \uplus \sigma:\left[n_{1}:\right.$ $\varepsilon]|\ldots|\left[n_{k}: \varepsilon\right] \mid P \longmapsto{ }^{*} P^{\prime}$. By Lemma 58, there exists $P_{1}$ such that $\left\{{ }^{M_{p^{\prime}}} / x_{p^{\prime}}\right\} \uplus \ldots \uplus\left\{{ }^{M_{1}} / x_{1}\right\} \uplus \sigma:$ $\left[n_{1}: S_{1}\right]|\ldots|\left[n_{k}: S_{k}\right] \mid P \longmapsto{ }^{*} P_{1}$ and $\llbracket P_{1} \rrbracket \equiv \llbracket P^{\prime} \rrbracket\left|\llbracket S_{1} \rrbracket_{n_{1}}\right| \ldots \mid \llbracket S_{k} \rrbracket_{n_{k}}$. Then, we can derive $\sigma: P \mid Q \longmapsto^{*} P^{\prime \prime}=\left(\nu m_{1}\right) \ldots\left(\nu m_{p}\right)\left(\nu x_{1}: M_{1}\right) \ldots\left(\nu x_{p^{\prime}}: M_{p^{\prime}}\right)\left(P_{1} \mid Q^{\prime}\right)$. Finally,

$$
\begin{aligned}
\llbracket P^{\prime \prime} \rrbracket & =\left(\nu m_{1}\right) \ldots\left(\nu m_{p}\right)\left(\llbracket P_{1} \rrbracket \mid \llbracket Q^{\prime} \rrbracket\right)\left\{{ }^{M_{p^{\prime}}} / x_{p^{\prime}}\right\} \ldots\left\{^{M_{1}} / x_{1}\right\} \\
& \equiv\left(\nu m_{1}\right) \ldots\left(\nu m_{p}\right)\left(\llbracket P^{\prime} \rrbracket\left|\llbracket S_{1} \rrbracket_{n_{1}}\right| \ldots\left|\llbracket S_{k} \rrbracket_{n_{k}}\right| \llbracket Q^{\prime} \rrbracket\right)\left\{{ }^{M_{p^{\prime}} / x_{p^{\prime}}}\right\} \ldots\left\{{ }^{M_{1}} / x_{1}\right\} \\
& \equiv \llbracket P^{\prime} \rrbracket \mid\left(\nu m_{1}\right) \ldots\left(\nu m_{p}\right)\left(\llbracket S_{1} \rrbracket_{n_{1}}|\ldots| \llbracket S_{k} \rrbracket_{n_{k}} \mid \llbracket Q^{\prime} \rrbracket\right)\left\{{ }^{M_{p^{\prime}}} / x_{p^{\prime}}\right\} \ldots\left\{{ }^{M_{1}} / x_{1}\right\} \\
& \equiv \llbracket P^{\prime} \rrbracket \mid \llbracket Q \rrbracket \\
& =\llbracket P^{\prime} \mid Q \rrbracket
\end{aligned}
$$

Finally, the next two lemmas are the missing lemmas from Section A.3, i.e. the inductive steps for parallel composition and channels.

$\mathrm{RR} \mathrm{n}^{\circ} 4350$ 
Lemma 60 If $\vdash P \mid Q:$ Valid, $f v(Q) \subseteq \operatorname{dom}(\sigma)$ and $\sigma: c l_{\sigma}(P) \longmapsto^{*} P^{\prime}$, then $\sigma:$ $c l_{\sigma}(P \mid Q) \longmapsto^{*} P^{\prime \prime}$ with $\llbracket P^{\prime \prime} \rrbracket \equiv \llbracket P^{\prime} \mid Q \rrbracket$.

Proof: Let us write $c l_{\sigma}(P)=\left[n_{1}: \varepsilon\right]|\ldots|\left[n_{k}: \varepsilon\right]\left|\left[m_{1}: \varepsilon\right]\right| \ldots\left|\left[m_{k^{\prime}}: \varepsilon\right]\right| c l(P)$ with $(f n(P) \cup f n(\sigma)) \backslash \operatorname{pr}(P)=\left\{n_{1}, \ldots, n_{k}, m_{1}, \ldots, m_{k^{\prime}}\right\}$ divided so that $Q \Downarrow_{1} n_{i}$ and $Q \Downarrow_{1} m_{j}$. Let $P_{0}=\left[m_{1}: \varepsilon\right]|\ldots|\left[m_{k^{\prime}}: \varepsilon\right] \mid \operatorname{cl}(P)$. We know that $\sigma:\left[n_{1}: \varepsilon\right]|\ldots|\left[n_{k}: \varepsilon\right] \mid P_{0} \longmapsto{ }^{*} P^{\prime}$. Since $\vdash P \mid Q:$ Valid, $\vdash P:$ Valid. By definition of $m_{j}, P \Downarrow_{1} m_{j}$, so that $\vdash P_{0}:$ Valid. Moreover, $P_{0} \Downarrow_{1} n_{i}, \vdash \operatorname{cl}(Q):$ Valid and $\operatorname{cl}(Q) \Downarrow_{1} n_{i}$ by Lemma 36. Using Lemma 59, there exists $P_{1}$ such that $\sigma: P_{0} \mid \operatorname{cl}(Q) \longmapsto^{*} P_{1}$ and $\llbracket P_{1} \rrbracket \equiv \llbracket P^{\prime}\left|c l(Q) \rrbracket \equiv \llbracket P^{\prime}\right| Q \rrbracket$ (using Lemma 41). We can write:

$$
\begin{aligned}
(f n(P \mid Q) \cup f n(\sigma)) \backslash p r(P \mid Q) & =(f n(P) \cup f n(Q) \cup f n(\sigma)) \backslash(\operatorname{pr}(P) \cup \operatorname{pr}(Q)) \\
& =((f n(P) \cup f n(\sigma)) \backslash \operatorname{pr}(P)) \backslash \operatorname{pr}(Q) \\
& \cup((f n(Q) \cup f n(\sigma)) \backslash \operatorname{pr}(Q)) \backslash \operatorname{pr}(P)
\end{aligned}
$$

Because $m_{j} \in(f n(P) \cup f n(\sigma)) \backslash p r(P)$ but $m_{j} \notin p r(Q)$, we have $m_{j} \in(f n(P \mid Q) \cup f n(\sigma)) \backslash p r(P \mid Q)$. This allows us to write $c l_{\sigma}(P \mid Q)=\left[p_{1}: \varepsilon\right]\left|\ldots\left[p_{l}: \varepsilon\right]\right|\left[m_{1}: \varepsilon\right]|\ldots|\left[m_{k^{\prime}}: \varepsilon\right]|\operatorname{cl}(P)| \operatorname{cl}(Q)=$ $\left[p_{1}: \varepsilon\right]\left|\ldots\left[p_{l}: \varepsilon\right]\right| P_{0} \mid c l(Q)$. And we can derive $\sigma: c l_{\sigma}(P \mid Q) \longmapsto{ }^{*} P^{\prime \prime}=\left[p_{1}: \varepsilon\right]\left|\ldots\left[p_{l}: \varepsilon\right]\right| P_{1}$. Finally, $\llbracket P^{\prime \prime} \rrbracket \equiv \llbracket P_{1} \rrbracket \equiv \llbracket P^{\prime} \mid Q \rrbracket$.

Lemma 61 If $\vdash\left[n: S \mid S^{\prime}\right]:$ Valid, $f v\left(S^{\prime}\right) \subseteq \operatorname{dom}(\sigma)$ and $\sigma: c l_{\sigma}([n: S]) \longmapsto^{*} P$, then $\sigma: c l_{\sigma}\left(\left[n: S \mid S^{\prime}\right]\right) \longmapsto^{*} P^{\prime}$ with $\llbracket P^{\prime} \rrbracket \equiv \llbracket P \rrbracket \mid \llbracket S^{\prime} \rrbracket_{n}$.

Proof: Let us write $c l_{\sigma}([n: S])=\left[n_{1}: \varepsilon\right]|\ldots|\left[n_{k}: \varepsilon\right] \mid[n: c l(S)]$ with $(f n([n: S]) \cup f n(\sigma)) \backslash$ $\operatorname{pr}([n: S])=\left\{n_{1}, \ldots, n_{k}\right\}$. By Lemma 56, $\sigma: \operatorname{cl}_{\sigma}([n: S])\left\{n+\operatorname{cl}\left(S^{\prime}\right)\right\}=\left[n_{1}: \varepsilon\right]|\ldots|\left[n_{k}:\right.$ $\varepsilon] \mid\left[n: \operatorname{cl}(S) \mid \operatorname{cl}\left(S^{\prime}\right)\right] \longmapsto^{*} P\left\{n \leftarrow \operatorname{cl}\left(S^{\prime}\right)\right\}$. Moreover, $\operatorname{pr}([n: S])=\operatorname{pr}\left(\left[n: S \mid S^{\prime}\right]\right)=\{n\}$. Then, $\left(f n\left(\left[n: S \mid S^{\prime}\right]\right) \cup f n(\sigma)\right) \backslash p r\left(\left[n: S \mid S^{\prime}\right]\right)=\left(\{n\} \cup f n(S) \cup f n\left(S^{\prime}\right) \cup f n(\sigma)\right) \backslash\{n\}=$ $\left\{n_{1}, \ldots, n_{k}\right\} \cup\left(f n\left(S^{\prime}\right) \backslash\{n\}\right) \supseteq\left\{n_{1}, \ldots, n_{k}\right\}$. We can derive $\sigma: c l_{\sigma}\left(\left[n: S \mid S^{\prime}\right]\right) \longmapsto^{*} P^{\prime}=$ $\left[m_{1}: \varepsilon\right]|\ldots|\left[m_{p}: \varepsilon\right] \mid P\left\{n+\operatorname{cl}\left(S^{\prime}\right)\right\}$. Since $\vdash[n: S]:$ Valid, $\vdash P:$ Valid by Lemma 36 and Proposition 6. Moreover, by Corollary 35 and Lemma 36, $\operatorname{pr}(P)=\operatorname{pr}([n: S])=\{n\}$. Finally, using Lemma $57, \llbracket P^{\prime} \rrbracket \equiv \llbracket P\left\{n+c l\left(S^{\prime}\right)\right\} \rrbracket \equiv \llbracket P \rrbracket\left|\llbracket c l\left(S^{\prime}\right) \rrbracket_{n} \equiv \llbracket P \rrbracket\right| \llbracket S^{\prime} \rrbracket_{n}$ (using Lemma 41). 


\section{A.6 Proof of Proposition 8}

Proposition 8 If $\sigma: P \longmapsto Q$, then $\llbracket P \rrbracket \sigma^{*} \stackrel{\equiv}{\longrightarrow} \llbracket Q \rrbracket \sigma^{*}$, where $\stackrel{\equiv}{\longrightarrow}$ is either $\equiv$ or $\longrightarrow$.

Proof: By induction on the derivation of $\sigma: P \longmapsto Q$.

( $\pi_{e s c}$ Red Subst Out) Suppose that $\sigma: \bar{x}\left\langle M^{\prime}\right\rangle \cdot P \longmapsto \bar{M}\left\langle M^{\prime}\right\rangle \cdot P$ with $x \sigma=M$. We have obviously $x \sigma^{*}=M \sigma^{*}$. Then, $\llbracket \bar{x}\left\langle M^{\prime}\right\rangle \cdot P \rrbracket \sigma^{*}=\overline{\left(x \sigma^{*}\right)}\left\langle M^{\prime} \sigma^{*}\right\rangle \cdot\left(\llbracket P \rrbracket \sigma^{*}\right)=\overline{\left(M \sigma^{*}\right)}\left\langle M^{\prime} \sigma^{*}\right\rangle \cdot\left(\llbracket P \rrbracket \sigma^{*}\right)=$ $\llbracket \bar{M}\left\langle M^{\prime}\right\rangle . P \rrbracket \sigma^{*}$.

( $\pi_{e s c}$ Red Subst In) Similar to ( $\pi_{\text {esc }}$ Red Subst Out).

( $\pi_{\text {esc }}$ Red Output) Suppose that $\sigma:[n: S] \mid \bar{n}\langle M\rangle . P \longmapsto[n: S \mid\langle M\rangle . P]$. We have $\llbracket[n: S]\left|\bar{n}\langle M\rangle \cdot P \rrbracket=\llbracket S \rrbracket_{n}\right| \bar{n}\langle M\rangle \cdot \llbracket P \rrbracket=\llbracket S \mid\langle M\rangle . P \rrbracket_{n}=\llbracket[n: S \mid\langle M\rangle . P] \rrbracket . \quad$ Then, $\llbracket[n: S] \mid \bar{n}\langle M\rangle . P \rrbracket \sigma^{*}=\llbracket[n: S \cup\{\langle M\rangle . P\}] \rrbracket \sigma^{*}$.

( $\pi_{e s c}$ Red Input) Similar to ( $\pi_{e s c}$ Red Output).

$\left(\pi_{e s c}\right.$ Red Comm) Suppose that $\sigma:[n: S \mid(\langle M\rangle \cdot P \mid(x) \cdot Q)] \longmapsto[n: S] \mid(P \mid(\nu x: M) Q)$ with $x \neq M$ (we also suppose $x \notin \operatorname{dom}(\sigma)$ ). We have:

$$
\begin{aligned}
& \llbracket\left[n: S \mid(\langle M\rangle \cdot P \mid(x) \cdot Q) \rrbracket \rrbracket \sigma^{*}\right. \\
= & \llbracket S \rrbracket_{n} \sigma^{*} \mid\left(\bar{n}\left\langle M \sigma^{*}\right\rangle \cdot\left(\llbracket P \rrbracket \sigma^{*}\right) \mid n(x) \cdot\left(\llbracket Q \rrbracket \sigma^{*}\right)\right) \\
\longrightarrow & \llbracket S \rrbracket_{n} \sigma^{*} \mid\left(\llbracket P \rrbracket \sigma^{*} \mid\left(\llbracket Q \rrbracket \sigma^{*}\right)\left\{{ }^{M \sigma^{*}} / x\right\}\right) \quad(\text { by }(\pi \text { Red Comm })) \\
= & \llbracket S \rrbracket_{n} \sigma^{*} \mid\left(\llbracket P \rrbracket \sigma^{*} \mid\left(\llbracket Q \rrbracket\left\{\left\{^{M} / x\right\}\right) \sigma^{*}\right) \quad\right. \text { (using Lemma 26) } \\
= & \llbracket[n: S] \mid(P \mid(\nu x: M) Q) \rrbracket \sigma^{*}
\end{aligned}
$$

( $\pi_{e s c}$ Red Par) Suppose that $\sigma: P\left|Q \longmapsto P^{\prime}\right| Q$ was derived from $\sigma: P \longmapsto P^{\prime}$. By induction

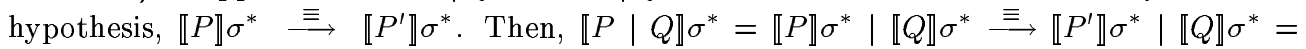
$\llbracket P^{\prime} \mid Q \rrbracket \sigma^{*}$ by ( $\pi$ Struct Par) or ( $\pi$ Red Par).

( $\pi_{e s c}$ Red Res) Suppose that $\sigma:(\nu n) P \longmapsto(\nu n) P^{\prime}$ was derived from $\sigma: P \longmapsto P^{\prime}$.

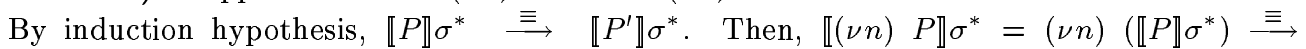
$(\nu n)\left(\llbracket P^{\prime} \rrbracket \sigma^{*}\right)=\llbracket(\nu n) P \rrbracket \sigma^{*}$ by ( $\pi$ Struct Res) or ( $\pi$ Red Res) (we can always suppose $n \notin \operatorname{im}(\sigma))$.

( $\pi_{e s c}$ Red Var) Suppose that $\sigma:(\nu x: M) P \longmapsto(\nu x: M) P^{\prime}$ was derived from $\left\{{ }^{M} / x\right\} \uplus \sigma:$ $P \longmapsto P^{\prime}$ with $x \notin \operatorname{dom}(\sigma)$. By induction hypothesis, we have $\llbracket P \rrbracket\left(\left\{{ }^{M} / x\right\} \uplus \sigma\right)^{*} \stackrel{\equiv}{\longrightarrow}$ $\llbracket Q \rrbracket\left(\left\{{ }^{M} / x\right\} \uplus \sigma\right)^{*}$. Then, $\llbracket(\nu x: M) P \rrbracket \sigma^{*}=\llbracket P \rrbracket\left\{{ }^{M} / x\right\} \sigma^{*}=\llbracket P \rrbracket\left(\left\{^{M} / x\right\} \uplus \sigma\right)^{*} \stackrel{\equiv}{\longrightarrow} \llbracket Q \rrbracket\left(\left\{{ }^{M} / x\right\} \uplus\right.$ $\sigma)^{*}=\llbracket Q \rrbracket\left\{{ }^{M} / x\right\} \sigma^{*}=\llbracket(\nu x: M) Q \rrbracket \sigma^{*}$, using Lemma 27 .

( $\pi_{e s c}$ Red Struct) Suppose that $\sigma: P \longmapsto Q$ was derived from $P \equiv P^{\prime}, \sigma: P^{\prime} \longmapsto Q^{\prime}$ and $Q^{\prime} \equiv Q$. By Lemma $42, \llbracket P \rrbracket \equiv \llbracket P^{\prime} \rrbracket$ and by Lemma $28, \llbracket P \rrbracket \sigma^{*} \equiv \llbracket P^{\prime} \rrbracket \sigma^{*}$. By induction hypothesis, $\llbracket P^{\prime} \rrbracket \sigma^{*} \equiv \llbracket Q^{\prime} \rrbracket \sigma^{*}$. Using Lemmas 42 and $28, \llbracket Q^{\prime} \rrbracket \sigma^{*} \equiv \llbracket Q \rrbracket \sigma^{*}$. Finally, we can derive $\llbracket P \rrbracket \sigma^{*} \stackrel{\equiv}{\longrightarrow} \llbracket Q \rrbracket \sigma^{*}$ by ( $\pi$ Struct Trans) or ( $\pi$ Red Struct). 


\section{A.7 Proof of Proposition 9}

Proposition 9 If $\llbracket P \rrbracket \sigma^{*} \longrightarrow Q, \vdash P:$ Valid and $f v(P) \subseteq \operatorname{dom}(\sigma)$, then there is a process $P^{\prime}$ such that $\sigma: c l_{\sigma}(P) \longmapsto^{+} P^{\prime}$ and $\llbracket P^{\prime} \rrbracket \sigma^{*} \equiv Q$.

Proof: By induction on the derivation of $\llbracket P \rrbracket \sigma^{*} \longrightarrow Q$, with a special treatment for $P=(\nu x$ : M) $P^{\prime}$ : since this part of the reasoning is common to all cases, we include it in the induction and we consider that $P$ is not of this form in all other cases.

$(\nu \mathbf{x}: \mathbf{M}) \mathbf{P}$ Suppose that $\llbracket(\nu x: M) P \rrbracket \sigma^{*} \longrightarrow Q, \vdash(\nu x: M) P:$ Valid and $f v((\nu x: M) P) \subseteq$ $\operatorname{dom}(\sigma)$. We also suppose $x \notin \operatorname{dom}(\sigma)$. We have $\llbracket P \rrbracket\left\{{ }^{M} / x\right\} \sigma^{*} \longrightarrow Q$. Let $\sigma^{\prime}=\left\{{ }^{M} / x\right\} \uplus \sigma$. By Lemma 27, $\sigma^{* *}=\left\{{ }^{M} / x\right\} \sigma^{*}$. So we have $\llbracket P \rrbracket \sigma^{\prime *} \longrightarrow Q, \vdash P:$ Valid (from $\vdash(\nu x:$ M) $P:$ Valid) and $f v(P) \subseteq \operatorname{dom}\left(\sigma^{\prime}\right)$. By induction hypothesis, there exists $P^{\prime}$ such that $\sigma^{\prime}: c l_{\sigma^{\prime}}(P) \longmapsto^{+} P^{\prime}$ and $\llbracket P^{\prime} \rrbracket \sigma^{\prime *} \equiv Q$. Then, we can derive $\sigma:(\nu x: M) c l_{\sigma^{\prime}}(P) \longmapsto^{+}$ $(\nu x: M) P^{\prime}$ by $\left(\pi_{e s c}\right.$ Red Var). By Lemma $45,(\nu x: M) c l_{\sigma^{\prime}}(P) \equiv c l_{\sigma}((\nu x: M) P)$, so we can derive $\sigma: c l_{\sigma}((\nu x: M) P) \longmapsto^{+}(\nu x: M) P^{\prime}$. And $\llbracket(\nu x: M) P^{\prime} \rrbracket \sigma^{*}=\llbracket P^{\prime} \rrbracket\left\{{ }^{M} /{ }_{x}\right\} \sigma^{*}=$ $\llbracket P^{\prime} \rrbracket \sigma^{\prime *} \equiv Q$.

( $\pi$ Red Comm) Suppose that $\llbracket P \rrbracket \sigma^{*}=\bar{m}\langle M\rangle \cdot P_{1}\left|n(x) . P_{2} \longrightarrow P_{1}\right| P_{2}\left\{{ }^{m} / x\right\}=Q, \vdash P:$ Valid and $f v(P) \subseteq \operatorname{dom}(\sigma)$. Necessarily, $\llbracket P \rrbracket=\overline{M_{1}}\left\langle M_{2}\right\rangle \cdot P_{3} \mid M_{3}(x) . P_{4}$ with $M_{1} \sigma^{*}=n, M_{2} \sigma^{*}=m$, $P_{3} \sigma^{*}=P_{1}, M_{3} \sigma^{*}=n$ and $P_{4} \sigma^{*}=P_{2}$ (we can always suppose $x \neq M_{2}$ and $x \notin \operatorname{dom}(\sigma)$ ). By Lemma 43 , there are two cases to consider.

- $P=\left[p: S \mid S^{\prime}\right]$ with $\llbracket S \rrbracket_{p}=\overline{M_{1}}\left\langle M_{2}\right\rangle \cdot P_{3}$ and $\llbracket S^{\prime} \rrbracket_{p}=M_{3}(x) . P_{4}$. The first assertion implies $M_{1}=p$ and $S=\left\langle M_{2}\right\rangle \cdot P_{5}$ with $\llbracket P_{5} \rrbracket=P_{3}$. The second assertion implies $M_{3}=p$ and $S^{\prime}=(x) \cdot P_{6}$ with $\llbracket P_{6} \rrbracket=P_{4}$. Then, we have $n=M_{1} \sigma^{*}=p \sigma^{*}=p$. To summarize, $P=\left[n:\left\langle M_{2}\right\rangle \cdot P_{5} \mid(x) \cdot P_{6}\right]$. Since $x \neq M_{2}$, we can derive $\sigma: P \longmapsto[n: \varepsilon] \mid\left(P_{5} \mid(\nu x\right.$ : $\left.\left.M_{2}\right) P_{6}\right)$ by $\left(\pi_{e s c} \operatorname{Red}\right.$ Comm). Then, $\sigma: c l_{\sigma}(P) \longmapsto P^{\prime}=\left[n_{1}: \varepsilon\right]|\ldots|\left[n_{k}:\right.$ $\varepsilon]|[n: \varepsilon]|\left(\operatorname{cl}\left(P_{5}\right) \mid\left(\nu x: M_{2}\right) \operatorname{cl}\left(P_{6}\right)\right)$. We have $\llbracket P^{\prime} \rrbracket \equiv \llbracket c l\left(P_{5}\right) \rrbracket \mid \llbracket c l\left(P_{6}\right) \rrbracket\left\{{ }^{M_{2}} / x\right\} \equiv$ $\llbracket P_{5} \rrbracket\left|\llbracket P_{6} \rrbracket\left\{{ }^{M_{2}} / x\right\}=P_{3}\right| P_{4}\left\{{ }^{M_{2}} / x\right\}$. Then, by Lemma $28, \llbracket P^{\prime} \rrbracket \sigma^{*} \equiv P_{3} \sigma^{*} \mid P_{4}\left\{{ }^{M_{2}} / x\right\} \sigma^{*}$. Using Lemma $26, \llbracket P^{\prime} \rrbracket \sigma^{*} \equiv P_{3} \sigma^{*}\left|P_{4} \sigma^{*}\left\{{ }^{M_{2} \sigma^{*}} / x\right\}=P_{1}\right| P_{2}\left\{{ }^{m} / x\right\}=Q$.

- $P=P_{5} \mid P_{6}$ with $\llbracket P_{5} \rrbracket=\overline{M_{1}}\left\langle M_{2}\right\rangle \cdot P_{3}$ and $\llbracket P_{6} \rrbracket=M_{3}(x) \cdot P_{4}$. There can be some variable restrictions in $P_{5}$ and $P_{6}$. We take all of them into account: $P_{5}=\left(\nu x_{1}\right.$ : $\left.N_{1}\right) \ldots\left(\nu x_{k}: N_{k}\right) P_{5}^{\prime}$ and $P_{6}=\left(\nu y_{1}: N_{1}^{\prime}\right) \ldots\left(\nu y_{k^{\prime}}: N_{k^{\prime}}^{\prime}\right) P_{6}^{\prime}$. We can always rename the variables in order to have the following assumptions: $x_{i} \notin \operatorname{dom}(\sigma) \cup$ $f v\left(P_{4}\right) \cup f v\left(P_{6}\right) \cup\left\{M_{3}, y_{1}, \ldots, y_{k^{\prime}}, N_{1}^{\prime}, \ldots, N_{k^{\prime}}^{\prime}\right\}$ and $y_{j} \notin \operatorname{dom}(\sigma) \cup f v\left(P_{3}\right) \cup f v\left(P_{5}^{\prime}\right) \cup$ $\left\{x, M_{2}, x_{1}, \ldots, x_{k}, N_{1}, \ldots, N_{k}\right\}$. Let $\sigma_{x}=\left\{{ }^{N_{k}} / x_{k}\right\} \ldots\left\{{ }^{N_{1}} / x_{1}\right\}, \sigma_{x}^{\prime}=\left\{{ }^{N_{k}} / x_{k}\right\} \uplus \ldots \uplus$ $\left\{{ }^{N_{1}} / x_{1}\right\}, \sigma_{y}=\left\{{ }^{N_{k^{\prime}}^{\prime}} / y_{k^{\prime}}\right\} \ldots\left\{{ }^{N_{1}^{\prime}} / y_{1}\right\}$ and $\sigma_{y}^{\prime}=\left\{{ }^{N_{k^{\prime}}^{\prime}} / y_{k^{\prime}}\right\} \uplus \ldots \uplus\left\{{ }^{N_{1}^{\prime}} / y_{1}\right\}$. Since $f v(P) \subseteq$ $\operatorname{dom}(\sigma), N_{i} \in \operatorname{Name} \cup \operatorname{dom}(\sigma) \cup\left\{x_{1}, \ldots, x_{i-1}\right\}$ and $N_{j}^{\prime} \in \operatorname{Name} \cup \operatorname{dom}(\sigma) \cup\left\{y_{1}, \ldots, y_{j-1}\right\}$. With the above conditions, we can prove by Lemma 27 that $\left(\sigma_{y}^{\prime} \uplus \sigma_{x}^{\prime} \uplus \sigma\right)^{*}=\sigma_{y} \sigma_{x} \sigma^{*}$. Moreover, $\sigma_{y} \sigma_{x}=\sigma_{x} \sigma_{y}$ easily. Then, we have $\llbracket P_{5}^{\prime} \rrbracket \sigma_{x}=\llbracket P_{5} \rrbracket=\overline{M_{1}}\left\langle M_{2}\right\rangle \cdot P_{3}$ and $\llbracket P_{6}^{\prime} \rrbracket \sigma_{y}=\llbracket P_{6} \rrbracket=M_{3}(x) \cdot P_{4}$. Then, $\llbracket P_{5}^{\prime} \rrbracket=\overline{M_{1}^{\prime}}\left\langle M_{2}^{\prime}\right\rangle \cdot P_{3}^{\prime}$ with $M_{1}^{\prime} \sigma_{x}=M_{1}, M_{2}^{\prime} \sigma_{x}=M_{2}$ and $P_{3}^{\prime} \sigma_{x}=P_{3}$, and $\llbracket P_{6}^{\prime} \rrbracket=M_{3}^{\prime}(x) . P_{4}^{\prime}$ with $M_{3}^{\prime} \sigma_{y}=M_{3}$ and $P_{4}^{\prime} \sigma_{y}=P_{4}$. Using Lemma 44, there are four cases to consider:

- $P_{5}^{\prime}=\left[p:\left\langle M_{2}^{\prime}\right\rangle \cdot P_{3}^{\prime \prime}\right]$ with $M_{1}^{\prime}=p$ and $\llbracket P_{3}^{\prime \prime} \rrbracket=P_{3}^{\prime}$, and $P_{6}^{\prime}=\left[p^{\prime}:(x) \cdot P_{4}^{\prime \prime}\right]$ with $M_{3}^{\prime}=p^{\prime}$ and $\llbracket P_{4}^{\prime \prime} \rrbracket=P_{4}^{\prime}$. In this case, $n=M_{1} \sigma^{*}=M_{1}^{\prime} \sigma_{x} \sigma^{*}=p \sigma_{x} \sigma^{*}=p$ and $n=M_{3} \sigma^{*}=M_{3}^{\prime} \sigma_{y} \sigma^{*}=p^{\prime} \sigma_{y} \sigma^{*}=p^{\prime}$. Then, $P_{5}^{\prime} \Downarrow_{1} n$ and $P_{6}^{\prime} \Downarrow_{1} n$. We can derive $P_{5} \Downarrow_{1} n$ and $P_{6} \Downarrow_{1} n$. Finally, $P \Downarrow_{2} n$, but this is in contradiction with $\vdash P$ : Valid. Consequently, this case is impossible. 
- $P_{5}^{\prime}=\left[p:\left\langle M_{2}^{\prime}\right\rangle \cdot P_{3}^{\prime \prime}\right]$ with $M_{1}^{\prime}=p$ and $\llbracket P_{3}^{\prime \prime} \rrbracket=P_{3}^{\prime}$, and $P_{6}^{\prime}=M_{3}^{\prime}(x) \cdot P_{4}^{\prime \prime}$ with $\llbracket P_{4}^{\prime \prime} \rrbracket=P_{4}^{\prime}$. In this case, $n=M_{1} \sigma^{*}=M_{1}^{\prime} \sigma_{x} \sigma^{*}=p \sigma_{x} \sigma^{*}=p$. We have $f v\left(P_{6}^{\prime}\right) \subseteq$ $\left\{y_{1}, \ldots, y_{k^{\prime}}\right\} \cup f v\left(P_{6}\right) \subseteq\left\{y_{1}, \ldots, y_{k^{\prime}}\right\} \cup f v(P) \subseteq \operatorname{dom}\left(\sigma_{x}^{\prime} \uplus \sigma_{y}^{\prime} \uplus \sigma\right)$. Moreover, $M_{3}^{\prime}\left(\sigma_{y}^{\prime} \uplus \sigma_{x}^{\prime} \uplus \sigma\right)^{*}=M_{3}^{\prime} \sigma_{y} \sigma_{x} \sigma^{*}=M_{3} \sigma_{x} \sigma^{*}=M_{3} \sigma^{*}=n$. Thus, by Lemma 38, we can derive $\sigma_{y}^{\prime} \uplus \sigma_{x}^{\prime} \uplus \sigma: P_{6}^{\prime} \longmapsto^{*} n(x)$. $P_{4}^{\prime \prime}$. With a similar reasoning, $f v\left(P_{5}^{\prime}\right) \subseteq$ $\operatorname{dom}\left(\sigma_{x}^{\prime} \uplus \sigma_{y}^{\prime} \uplus \sigma\right)$. We can derive:

$$
\begin{array}{lll} 
& \sigma_{y}^{\prime} \uplus \sigma_{x}^{\prime} \uplus \sigma: P_{5}^{\prime} \mid P_{6}^{\prime} \\
\longmapsto * & {\left[n:\left\langle M_{2}^{\prime}\right\rangle \cdot P_{3}^{\prime \prime}\right] \mid n(x) \cdot P_{4}^{\prime \prime} \quad\left(\text { by }\left(\pi_{e s c} \text { Red Par }\right)\right)} \\
\longmapsto & {\left[n:\left\langle M_{2}^{\prime}\right\rangle \cdot P_{3}^{\prime \prime} \mid(x) \cdot P_{4}^{\prime \prime}\right] \quad\left(\text { by }\left(\pi_{e s c} \text { Red Input }\right)\right)} \\
\longmapsto & {[n: \varepsilon] \mid\left(P_{3}^{\prime \prime} \mid\left(\nu x: M_{2}^{\prime}\right) P_{4}^{\prime \prime}\right) \quad\left(\text { by }\left(\pi_{e s c} \text { Red Comm }\right)\right)}
\end{array}
$$

With the above conditions, we have $P=P_{5} \mid P_{6} \equiv\left(\nu x_{1}: N_{1}\right) \ldots\left(\nu x_{k}: N_{k}\right)\left(P_{5}^{\prime} \mid P_{6}\right) \equiv$ $\left(\nu x_{1}: N_{1}\right) \ldots\left(\nu x_{k}: N_{k}\right)\left(\nu y_{1}: N_{1}^{\prime}\right) \ldots\left(\nu y_{k^{\prime}}: N_{k^{\prime}}^{\prime}\right)\left(P_{5}^{\prime} \mid P_{6}^{\prime}\right)$. Using $\left(\pi_{e s c} \operatorname{Red} \operatorname{Var}\right)$ and ( $\pi_{e s c}$ Red Struct), we can derive $\sigma: P \longmapsto^{+}\left(\nu x_{1}: N_{1}\right) \ldots\left(\nu x_{k}: N_{k}\right)\left(\nu y_{1}\right.$ : $\left.N_{1}^{\prime}\right) \ldots\left(\nu y_{k^{\prime}}: N_{k^{\prime}}^{\prime}\right)\left([n: \varepsilon]\left|P_{3}^{\prime \prime}\right|\left(\nu x: M_{2}^{\prime}\right) P_{4}^{\prime \prime}\right)$. Finally, $\sigma: c l_{\sigma}(P) \longmapsto^{+} P^{\prime}=$ $\left[n_{1}: \varepsilon\right]|\ldots|\left[n_{r}: \varepsilon\right] \mid\left(\nu x_{1}: N_{1}\right) \ldots\left(\nu x_{k}: N_{k}\right)\left(\nu y_{1}: N_{1}^{\prime}\right) \ldots\left(\nu y_{k^{\prime}}: N_{k^{\prime}}^{\prime}\right)([n:$ $\left.\varepsilon]\left|c l\left(P_{3}^{\prime \prime}\right)\right|\left(\nu x: M_{2}^{\prime}\right) \operatorname{cl}\left(P_{4}^{\prime \prime}\right)\right)$. Then,

$$
\begin{aligned}
\llbracket P^{\prime} \rrbracket & \equiv\left(\llbracket c l\left(P_{3}^{\prime \prime}\right) \rrbracket \mid \llbracket c l\left(P_{4}^{\prime \prime}\right) \rrbracket\left\{\left\{^{M_{2}^{\prime}} / x\right\}\right) \sigma_{y} \sigma_{x}\right. \\
& \equiv\left(\llbracket P_{3}^{\prime \prime} \rrbracket \mid \llbracket P_{4}^{\prime \prime} \rrbracket\left\{{ }^{M_{2}^{\prime}} / x\right\}\right) \sigma_{y} \sigma_{x} \\
& \equiv P_{3}^{\prime} \sigma_{y} \sigma_{x} \mid P_{4}^{\prime}\left\{{ }^{M_{2}^{\prime}} / x\right\} \sigma_{y} \sigma_{x} \\
& =P_{3}^{\prime} \sigma_{x} \sigma_{y} \mid P_{4}^{\prime} \sigma_{y} \sigma_{x}\left\{{ }_{2}^{\prime} \sigma_{x} \sigma_{y} / x\right\} \\
& =P_{3} \sigma_{y} \mid P_{4} \sigma_{x}\left\{{ }^{M_{2} \sigma_{y}} / x\right\} \\
& =P_{3} \mid P_{4}\left\{{ }^{M_{2}} /{ }_{x}\right\} \\
\llbracket P^{\prime} \rrbracket \sigma^{*} & \equiv P_{3} \sigma^{*} \mid P_{4} \sigma^{*}\left\{{ }^{M_{2} \sigma^{*}} / x\right\} \\
& =P_{1} \mid P_{2}\left\{{ }^{m} / x\right\} \\
& =Q
\end{aligned}
$$

- $P_{5}^{\prime}=\overline{M_{1}^{\prime}}\left\langle M_{2}^{\prime}\right\rangle \cdot P_{3}^{\prime \prime}$ with $\llbracket P_{3}^{\prime \prime} \rrbracket=P_{3}^{\prime}$, and $P_{6}^{\prime}=\left[p:(x) \cdot P_{4}^{\prime \prime}\right]$ with $M_{3}^{\prime}=p$ and $\llbracket P_{4}^{\prime \prime} \rrbracket=P_{4}^{\prime}$. This case is very similar to the last one: we just need to exchange the role of $P_{5}$ and $P_{6}$.

- $P_{5}^{\prime}=\overline{M_{1}^{\prime}}\left\langle M_{2}^{\prime}\right\rangle \cdot P_{3}^{\prime \prime}$ with $\llbracket P_{3}^{\prime \prime} \rrbracket=P_{3}^{\prime}$, and $P_{6}^{\prime}=M_{3}^{\prime}(x) \cdot P_{4}^{\prime \prime}$ with $\llbracket P_{4}^{\prime \prime} \rrbracket=P_{4}^{\prime}$. The reasoning is very similar, except that we have to introduce the channel $n$ explicitely. We do not detail the side conditions. From $M_{1}^{\prime}\left(\sigma_{y}^{\prime} \uplus \sigma_{x}^{\prime} \uplus \sigma\right)^{*}=n$ and Lemma 38, we derive $\sigma_{y}^{\prime} \uplus \sigma_{x}^{\prime} \uplus \sigma: P_{5}^{\prime} \longmapsto \bar{n}\left\langle M_{2}^{\prime}\right\rangle \cdot P_{3}^{\prime \prime}$. From $M_{3}^{\prime}\left(\sigma_{y}^{\prime} \uplus \sigma_{x}^{\prime} \uplus \sigma\right)^{*}=n$ and Lemma 38, we derive $\sigma_{y}^{\prime} \uplus \sigma_{x}^{\prime} \uplus \sigma: P_{6}^{\prime} \longmapsto n(x)$. $P_{4}^{\prime \prime}$. Then,

$$
\begin{aligned}
& \sigma_{y}^{\prime} \uplus \sigma_{x}^{\prime} \uplus \sigma:[n: \varepsilon]\left|P_{5}^{\prime}\right| P_{6}^{\prime} \quad \longmapsto^{*} \quad[n: \varepsilon]\left|\bar{n}\left\langle M_{2}^{\prime}\right\rangle \cdot P_{3}^{\prime \prime}\right| n(x) . P_{4}^{\prime \prime} \\
& \longmapsto \quad\left[n: \varepsilon \mid\left\langle M_{2}^{\prime}\right\rangle \cdot P_{3}^{\prime \prime}\right] \mid n(x) \cdot P_{4}^{\prime \prime} \\
& \longmapsto \quad\left[n: \varepsilon\left|\left\langle M_{2}^{\prime}\right\rangle \cdot P_{3}^{\prime \prime}\right|(x) \cdot P_{4}^{\prime \prime}\right] \\
& \longmapsto \quad[n: \varepsilon]\left|P_{3}^{\prime \prime}\right|\left(\nu x: M_{2}^{\prime}\right) P_{4}^{\prime \prime}
\end{aligned}
$$


Then, $\sigma:[n: \varepsilon] \mid P \longmapsto{ }^{+}\left(\nu x_{1}: N_{1}\right) \ldots\left(\nu x_{k}: N_{k}\right)\left(\nu y_{1}: N_{1}^{\prime}\right) \ldots\left(\nu y_{k^{\prime}}: N_{k^{\prime}}^{\prime}\right)([n:$ $\left.\varepsilon]\left|P_{3}^{\prime \prime}\right|\left(\nu x: M_{2}^{\prime}\right) P_{4}^{\prime \prime}\right)$. Since $\vdash P:$ Valid, $P_{3}^{\prime \prime} \Downarrow_{1} n$ and $P_{4}^{\prime \prime} \Downarrow_{1} n$. Consequently, $P \Downarrow_{1} n$ and $n \in(f n(P) \cup f n(\sigma)) \backslash p r(P)$. Finally, $\sigma: c l_{\sigma}(P)=\left[n_{1}: \varepsilon\right]|\ldots|\left[n_{r}:\right.$ $\varepsilon]|[n: \varepsilon]| \operatorname{cl}(P) \longmapsto^{+} P^{\prime}=\left[n_{1}: \varepsilon\right]|\ldots|\left[n_{r}: \varepsilon\right] \mid\left(\nu x_{1}: N_{1}\right) \ldots\left(\nu x_{k}:\right.$ $\left.N_{k}\right)\left(\nu y_{1}: N_{1}^{\prime}\right) \ldots\left(\nu y_{k^{\prime}}: N_{k^{\prime}}^{\prime}\right)\left([n: \varepsilon]\left|\operatorname{cl}\left(P_{3}^{\prime \prime}\right)\right|\left(\nu x: M_{2}^{\prime}\right) \operatorname{cl}\left(P_{4}^{\prime \prime}\right)\right)$. Checking the condition $\llbracket P \rrbracket \sigma^{*} \equiv Q$ is the same as above.

( $\pi$ Red Par) Suppose that $\llbracket P \rrbracket \sigma^{*}=P_{1}\left|Q \quad \longrightarrow P_{2}\right| Q$ was derived from $P_{1} \longrightarrow P_{2}$, and that $\vdash P:$ Valid and $f v(P) \subseteq \operatorname{dom}(\sigma)$. Necessarily, $\llbracket P \rrbracket=P_{3} \mid Q_{1}$ with $P_{3} \sigma^{*}=P_{1}$ and $Q_{1} \sigma^{*}=Q$. By Lemma 43 , there are two cases to consider.

- $P=\left[n: S \mid S^{\prime}\right]$ with $\llbracket S \rrbracket_{n}=P_{3}$ and $\llbracket S^{\prime} \rrbracket_{n}=Q_{1}$. Then, $P_{1}=\llbracket S \rrbracket_{n} \sigma^{*}=\llbracket[n: S] \rrbracket \sigma^{*}$ and $P_{1} \longrightarrow P_{2}$. From $\vdash P:$ Valid, we get $\vdash[n: S]: \operatorname{Valid}$, and from $f v(P) \subseteq \operatorname{dom}(\sigma)$, we get $f v([n: S]) \subseteq \operatorname{dom}(\sigma)$. By induction hypothesis, there is a process $P^{\prime \prime}$ such that $\sigma: c l_{\sigma}([n: S]) \longmapsto{ }^{+} P^{\prime \prime}$ and $\llbracket P^{\prime \prime} \rrbracket \sigma^{*} \equiv P_{2}$. By Lemma 61, since $\vdash P:$ Valid and $f v\left(S^{\prime}\right) \subseteq \operatorname{dom}(\sigma)$, there is a process $P^{\prime}$ such that $\sigma: c_{\sigma}(P) \longmapsto P^{+} P^{\prime}$ and $\llbracket P^{\prime} \rrbracket \equiv \llbracket P^{\prime \prime} \rrbracket\left|\llbracket S^{\prime} \rrbracket_{n}=\llbracket P^{\prime \prime} \rrbracket\right| Q_{1}$. Finally, $\llbracket P^{\prime} \rrbracket \sigma^{*} \equiv \llbracket P^{\prime \prime} \rrbracket \sigma^{*}\left|Q_{1} \sigma^{*} \equiv P_{2}\right| Q$.

- $P=P_{4} \mid Q_{2}$ with $\llbracket P_{4} \rrbracket=P_{3}$ and $\llbracket Q_{2} \rrbracket=Q_{1}$. Then, $P_{1}=\llbracket P_{4} \rrbracket \sigma^{*} \longrightarrow P_{2}$. Since $\vdash P:$ Valid, we get $\vdash P_{4}:$ Valid. By induction hypothesis, there is a process $P_{4}^{\prime}$ such that $\sigma: c l_{\sigma}\left(P_{4}\right) \longmapsto^{+} P_{4}^{\prime}$ and $\llbracket P_{4}^{\prime} \rrbracket \sigma^{*} \equiv P_{2}$. By Lemma 60 , there is $P^{\prime}$ such that $\sigma: c_{\sigma}\left(P_{4} \mid Q_{2}\right) \longmapsto^{+} P^{\prime}$ and $\llbracket P^{\prime} \rrbracket \equiv \llbracket P_{4}^{\prime} \mid Q_{2} \rrbracket$. Finally, $\sigma: c l_{\sigma}(P) \longmapsto^{+} P^{\prime}$ and $\llbracket P^{\prime} \rrbracket \sigma^{*} \equiv \llbracket P_{4}^{\prime} \rrbracket \sigma^{*}\left|\llbracket Q_{2} \rrbracket \sigma^{*} \equiv P_{2}\right| Q_{1} \sigma^{*}=P_{2} \mid Q$.

( $\pi$ Red Res) Suppose that $\llbracket P \rrbracket \sigma^{*}=(\nu n) P_{0} \longrightarrow(\nu n) Q_{0}=Q$ was derived from $P_{0} \longrightarrow Q_{0}$, and that $\vdash P:$ Valid and $f v(P) \subseteq \operatorname{dom}(\sigma)$. Necessarily, $P=(\nu n) P_{1}$ with $\llbracket P_{1} \rrbracket \sigma^{*}=P_{0}$ and $n \notin f n(\sigma)$. We easily get that $\vdash P_{1}:$ Valid. From $\llbracket P_{1} \rrbracket \sigma^{*} \longrightarrow Q_{0}$, we deduce by induction hypothesis that there exists $P_{1}^{\prime}$ such that $\sigma: c l_{\sigma}\left(P_{1}\right) \longmapsto P^{+} P_{1}^{\prime}$ and $\llbracket P_{1}^{\prime} \rrbracket \sigma^{*} \equiv Q_{0}$. We consider two cases:

- Suppose $n \in f n(P)$. We can derive $\sigma:(\nu n) c l_{\sigma}\left(P_{1}\right) \longmapsto{ }^{+} P^{\prime}=(\nu n) P_{1}^{\prime}$ by $\left(\pi_{e s c}\right.$ Red Res). By Lemma 47, $(\nu n) c l_{\sigma}\left(P_{1}\right) \equiv c l_{\sigma}(P)$, so that we can derive $\sigma: c l_{\sigma}(P) \longmapsto P^{\prime}$. Finally, $\llbracket P^{\prime} \rrbracket \sigma^{*}=(\nu n) \llbracket P_{1}^{\prime} \rrbracket \sigma^{*} \equiv(\nu n) Q_{0}=Q$.

- Suppose $n \notin f n(P)$. We can derive $\sigma:(\nu n)\left([n: \varepsilon] \mid c l_{\sigma}\left(P_{1}\right)\right) \longmapsto^{+} P^{\prime}=(\nu n)([n$ : $\left.\varepsilon] \mid P_{1}^{\prime}\right)$ by $\left(\pi_{e s c}\right.$ Red Par) and $\left(\pi_{e s c}\right.$ Red Res). By Lemma 47, $(\nu n)\left([n: \varepsilon] \mid c l_{\sigma}\left(P_{1}\right)\right) \equiv$ $c l_{\sigma}(P)$, so that we can derive $\sigma: c_{\sigma}(P) \longmapsto{ }^{+} P^{\prime}$. Finally, $\llbracket P^{\prime} \rrbracket \sigma^{*} \equiv(\nu n) \llbracket P_{1}^{\prime} \rrbracket \sigma^{*} \equiv$ $(\nu n) Q_{0}=Q$.

( $\pi$ Red Struct) $\quad$ Suppose that $\llbracket P \rrbracket \sigma^{*} \quad \longrightarrow \quad Q$ was derived from $\llbracket P \rrbracket \sigma^{*} \equiv P_{1}, P_{1} \longrightarrow Q_{1}$ and $Q_{1} \equiv Q$, and that $\vdash P$ : Valid and $f v(P) \subseteq \operatorname{dom}(\sigma)$. By Lemma 29, there exists $P_{2}$ such that $\llbracket P \rrbracket \equiv P_{2}$ and $P_{1}=P_{2} \sigma^{*}$. Then, by Lemma 54, there exists $P_{2}^{\prime}$ such that $P_{2}=\llbracket P_{2}^{\prime} \rrbracket$ and $P_{2}^{\prime} \preccurlyeq P$. By Lemma 53, we have $c l(P) \equiv\left[n_{1}: \varepsilon\right]|\ldots|\left[n_{k}: \varepsilon\right] \mid \operatorname{cl}\left(P_{2}^{\prime}\right)$ with $\left\{n_{1}, \ldots, n_{k}\right\}=\operatorname{pr}(P) \backslash \operatorname{pr}\left(P_{2}^{\prime}\right)$. Let $\left(f n\left(P_{2}^{\prime}\right) \cup f n(\sigma)\right) \backslash p r\left(P_{2}^{\prime}\right)=\left\{n_{i_{1}}, \ldots, n_{i_{k^{\prime}}}, m_{1}, \ldots, m_{l}\right\}$ with $n_{i} \neq m_{j}$. Then, we have:

$$
\begin{array}{rlr} 
& (f n(P) \cup f n(\sigma)) \backslash p r(P) \\
\supseteq & \left(f n\left(P_{2}^{\prime}\right) \cup f n(\sigma)\right) \backslash \operatorname{pr}(P) \quad \text { using Lemma 49 } \\
= & \left(f n\left(P_{2}^{\prime}\right) \cup f n(\sigma)\right) \backslash\left(\operatorname{pr}\left(P_{2}^{\prime}\right) \cup\left\{n_{1}, \ldots, n_{k}\right\}\right) \quad \text { using Corollary } 51 \\
= & \left(\left(f n\left(P_{2}^{\prime}\right) \cup f n(\sigma)\right) \backslash \operatorname{pr}\left(P_{2}^{\prime}\right)\right) \backslash\left\{n_{1}, \ldots, n_{k}\right\}
\end{array}
$$

INRIA 


$$
=\left\{m_{1}, \ldots, m_{l}\right\}
$$

Let us write $(f n(P) \cup f n(\sigma)) \backslash p r(P)=\left\{m_{1}, \ldots, m_{l}, p_{1}, \ldots, p_{l^{\prime}}\right\}$ (we have $p_{j} \neq n_{i}$ because $n_{i} \in \operatorname{pr}(P)$ and $\left.p_{j} \notin \operatorname{pr}(P)\right)$. We have:

$$
\begin{aligned}
c l_{\sigma}(P) \equiv & {\left[p_{1}: \varepsilon\right]|\ldots|\left[p_{l^{\prime}}: \varepsilon\right]\left|\left[m_{1}: \varepsilon\right]\right| \ldots\left|\left[m_{l}: \varepsilon\right]\right| c l(P) } \\
\equiv & {\left[p_{1}: \varepsilon\right]|\ldots|\left[p_{l^{\prime}}: \varepsilon\right]\left|\left[m_{1}: \varepsilon\right]\right| \ldots \mid\left[m_{l}: \varepsilon\right] } \\
& \left|\left[n_{1}: \varepsilon\right]\right| \ldots\left|\left[n_{k}: \varepsilon\right]\right| c l\left(P_{2}^{\prime}\right) \quad \text { using Lemma } 53 \\
\equiv & {\left[p_{1}: \varepsilon\right]|\ldots|\left[p_{l^{\prime}}: \varepsilon\right]\left|\left[n_{j_{1}}: \varepsilon\right]\right| \ldots\left|\left[n_{j_{k-k^{\prime}}}: \varepsilon\right]\right| c l_{\sigma}\left(P_{2}^{\prime}\right) }
\end{aligned}
$$

where $\left\{i_{1}, \ldots, i_{k^{\prime}}\right\}$ and $\left\{j_{1}, \ldots, j_{k-k^{\prime}}\right\}$ form a partition of $\{1, \ldots, k\}$. Since $\vdash P:$ Valid, $\vdash P_{2}^{\prime}:$ Valid by Lemma 52; and $f v\left(P_{2}^{\prime}\right)=f v(P) \subseteq \operatorname{dom}(\sigma)$ by Lemma 49. By induction hypothesis, there is a process $P_{3}$ such that $\sigma: P_{1}=\llbracket P_{2}^{\prime} \rrbracket \sigma^{*} \longmapsto{ }^{+} P_{3}$ and $\llbracket P_{3} \rrbracket \sigma^{*} \equiv Q_{1}$. We can derive $\sigma: c l_{\sigma}(P) \longmapsto P^{+}=\left[p_{1}: \varepsilon\right]|\ldots|\left[p_{l^{\prime}}: \varepsilon\right]\left|\left[n_{j_{1}}: \varepsilon\right]\right| \ldots\left|\left[n_{j_{k-k^{\prime}}}: \varepsilon\right]\right| P_{3}$. And we have $\llbracket P^{\prime} \rrbracket \sigma^{*} \equiv \llbracket P_{3} \rrbracket \sigma^{*} \equiv Q_{1} \equiv Q$. 


\section{A.8 Observational Equivalence}

This Section gives the proof for the full adequacy result of Proposition 12.

\section{A.8.1 Preliminary Lemmas}

Lemma 62 In the $\pi$-calculus,

- If $P \downarrow n$, then $n \in f n(P)$.

- If $P \downarrow x$, then $x \in f v(P)$.

Proof: By induction on the derivations of $P \downarrow n$ and $P \downarrow x$.

Lemma 63 In the $\pi$-calculus, if $P \equiv Q$, then $P \downarrow M \Leftrightarrow Q \downarrow M$.

Proof: By induction on the derivation of $P \equiv Q$, using Lemma 62 .

Lemma 64 In the $\pi$-calculus,

- If $P \downarrow M$, then $P \sigma \downarrow M \sigma$.

- If $P \sigma \downarrow M$, then there exists $M^{\prime}$ such that $P \downarrow M^{\prime}$ and $M^{\prime} \sigma=M$.

Proof: By induction on the derivations of $P \downarrow M$ and $P \sigma \downarrow M$.

\section{A.8.2 Proof of Proposition 12}

Proposition 12 For a process $P$ in the $\pi_{e s c}$-calculus, $P \downarrow M \Leftrightarrow \llbracket P \rrbracket \downarrow M$.

Proof: We prove the two implications separately.

$\mathbf{P} \downarrow \mathbf{M} \Rightarrow \llbracket \mathbf{P} \rrbracket \downarrow \mathbf{M} \quad$ By induction on the derivation of $P \downarrow M$ :

(Obs Res) Suppose that $(\nu n) P \downarrow M$ was derived from $P \downarrow M$ with $n \neq M$. By induction hypothesis, $\llbracket P \rrbracket \downarrow M$. We can derive $(\nu n) \llbracket P \rrbracket \downarrow M$ by (Obs Res), that is to say $\llbracket(\nu n) P \rrbracket \downarrow M$.

(Obs ParL) Suppose that $P \mid Q \downarrow M$ was derived from $P \downarrow M$. by induction hypothesis, $\llbracket P \rrbracket \downarrow M$. We can derive $\llbracket P \rrbracket \mid \llbracket Q \rrbracket \downarrow M$ by (Obs ParL), that is to say $\llbracket P \mid Q \rrbracket \downarrow M$.

(Obs ParR) Similar to (Obs ParL).

(Obs Repl) Suppose that $! P \downarrow M$ was derived from $P \downarrow M$. By induction, hypothesis, $\llbracket P \rrbracket \downarrow M$. We can derive $! \llbracket P \rrbracket \downarrow M$ by (Obs Repl), that is to say $\llbracket ! P \rrbracket \downarrow M$.

(Obs Output) Suppose that $\bar{M}\left\langle M^{\prime}\right\rangle \cdot P \downarrow M$. We can always derive $\bar{M}\left\langle M^{\prime}\right\rangle \cdot \llbracket P \rrbracket \downarrow M$ by (Obs Output), that is to say $\llbracket \bar{M}\left\langle M^{\prime}\right\rangle \cdot P \rrbracket \downarrow M$.

(Obs Input) Similar to (Obs Output).

INRIA 
(Obs Channel) Suppose that $[n: S] \downarrow n$ was derived with the condition $S \not \equiv \varepsilon$. $S$ must contain an abstraction of the form $\langle M\rangle \cdot P$ or $(x) . P$. Then, $\llbracket[n: S] \rrbracket=\llbracket S \rrbracket_{n}$ must

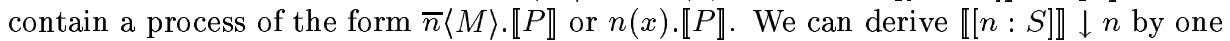
application of (Obs Output) or (Obs Input), and many applications of (Obs ParL) or (Obs ParR).

(Obs $\left.\operatorname{Var}_{1}\right)$ Suppose that $\left(\nu x: M^{\prime}\right) P \downarrow M$ was derived from $P \downarrow M$ with $x \neq M$. By induction hypothesis, $\llbracket P \rrbracket \downarrow M$. By Lemma $64, \llbracket P \rrbracket\left\{{ }^{M^{\prime}} / x\right\} \downarrow M\left\{^{M^{\prime}} / x\right\}$, that is to say $\llbracket\left(\nu x: M^{\prime}\right) P \rrbracket \downarrow M$ since $x \neq M$.

(Obs $\operatorname{Var}_{2}$ ) Suppose that $(\nu x: M) P \downarrow M$ was derived from $P \downarrow x$. By induction hypothesis, $\llbracket P \rrbracket \downarrow x$. By Lemma $64, \llbracket P \rrbracket\left\{{ }^{M} / x\right\} \downarrow x\left\{{ }^{M} / x\right\}$, that is to say $\llbracket(\nu x: M) P \rrbracket \downarrow M$.

$\llbracket \mathbf{P} \rrbracket \downarrow \mathbf{M} \Rightarrow \mathbf{P} \downarrow \mathbf{M} \quad$ By induction on the structure of $P$ :

- Suppose that $(\nu n) P \rrbracket \downarrow M$, that is to say $(\nu n) \llbracket P \rrbracket \downarrow M$. This must have been derived from $\llbracket P \rrbracket \downarrow M$ by (Obs Res) with $n \neq M$. By induction hypothesis, $P \downarrow M$ and we can derive $(\nu n) P \downarrow M$ by (Obs Res).

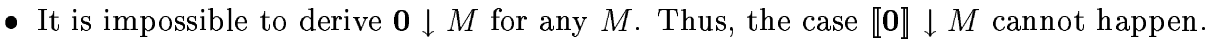

- Suppose that $\llbracket P \mid Q \rrbracket \downarrow M$, that is to say $\llbracket P \rrbracket \mid \llbracket Q \rrbracket \downarrow M$. This must have been derived from $\llbracket P \rrbracket \downarrow M$ or $\llbracket Q \rrbracket \downarrow M$ by (Obs ParL) or (Obs ParR). In the former case, $P \downarrow M$ by induction hypothesis, and $P \mid Q \downarrow M$ by (Obs ParL). The latter case is similar.

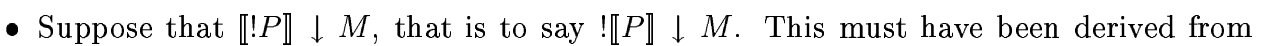
$\llbracket P \rrbracket \downarrow M$ by (Obs Repl). By induction hypothesis, $P \downarrow M$, and we can derive $! P \downarrow M$ by (Obs Repl).

- Suppose that $\llbracket \overline{M^{\prime}}\left\langle M^{\prime \prime}\right\rangle . P \rrbracket \downarrow M$, that is to say $\overline{M^{\prime}}\left\langle M^{\prime \prime}\right\rangle . \llbracket P \rrbracket \downarrow M$. This must have been derived by (Obs Output) and necessarily $M^{\prime}=M$. Then, we can derive $\overline{M^{\prime}}\left\langle M^{\prime \prime}\right\rangle . P \downarrow M$ by (Obs Output).

- The case of $\llbracket M^{\prime}(x) . P \rrbracket \downarrow M$ is very similar to the one of $\llbracket \overline{M^{\prime}}\left\langle M^{\prime \prime}\right\rangle . P \rrbracket \downarrow M$.

- Suppose that $\llbracket[n: S] \rrbracket \downarrow M$, that is to say $\llbracket S \rrbracket_{n} \downarrow M$. If $S \equiv \varepsilon$, this implies $\mathbf{0} \downarrow M$ by Lemma 63 , which is impossible. Thus, we must have $S \not \equiv \varepsilon$. Since $\llbracket S \rrbracket_{n} \downarrow M$ must have been derived by applications of the rules (Obs ParL) and (Obs ParR), and one application of the rule (Obs Output) or (Obs Input), $S \rrbracket_{n}$ must contain a process of the form $\bar{M}\left\langle M^{\prime}\right\rangle . P$ or $M(x) . P$, and necessarily, $n=M$. Then, $[n: S] \downarrow M$ by (Obs Channel).

- Suppose that $\llbracket\left(\nu x: M^{\prime}\right) P \rrbracket \downarrow M$, that is to say $\llbracket P \rrbracket\left\{{ }^{M^{\prime}} / x\right\} \downarrow M$. Note that we can always suppose $x \neq M$. By Lemma 64 , there is $M^{\prime \prime}$ such that $\llbracket P \rrbracket \downarrow M^{\prime \prime}$ and $M^{\prime \prime}\left\{{ }^{M^{\prime}} / x\right\}=M$. By induction hypothesis, $P \downarrow M^{\prime \prime}$. There are two cases to consider. If $M^{\prime \prime}=M$, we can derive $\left(\nu x: M^{\prime}\right) P \downarrow M$ by (Obs $\left.\operatorname{Var}_{1}\right)$ since $M \neq x$. Otherwise, we must have $M^{\prime \prime}=x$ and $M^{\prime}=M$. Then, we can derive $\left(\nu x: M^{\prime}\right) P \downarrow M$ by $\left(\mathrm{Obs}_{\operatorname{Var}_{2}}\right)$. 


\section{A.9 Encoding in Pure Ambients}

This Section gives the correctness proofs for the encoding of $\pi_{e s c}$ into pure ambients. These are facilitated by the similarity between the $\pi_{e s c}$-calculus and the encoding mechanism.

Lemma $65 f n(P)=f n(\{\{P\}) \backslash\{$ read, write, enter $\}$, and $f v(P)=f v(\{P\}\})$ (we always implicitly suppose that $P$ does not contain the special names read, write and enter).

Proof: By induction on the structure of $P$.

Lemma 66 If $P \equiv Q$, then $\{\{P\} \equiv\{\{Q\}$.

Proof: By induction on the derivation of $P \equiv Q$, using Lemma 65 .

A reduction in $\pi_{e s c}$ is simulated by exactly one principal reduction and many auxiliary reductions in pure ambients:

Proposition 18 If $\sigma: P \longmapsto Q$, then $\{\{\sigma, P\} \stackrel{p r}{\longrightarrow} \stackrel{a u x *}{\hookrightarrow}\{[\sigma, Q\}$.

Proof: By induction on the derivation of $\sigma: P \longmapsto Q$.

( $\pi_{e s c}$ Red Subst Out) See the main text.

( $\pi_{e s c}$ Red Output) Suppose that $\sigma:[n: S] \mid \bar{n}\langle M\rangle . P \longmapsto[n: S \mid\langle M\rangle . P]$. If we name $p_{1}, \ldots, p_{k}$ the fresh names in $S$, and if we choose them as well as $p$ to avoid interferences, we have:

$$
\begin{aligned}
& \{[n: S] \mid \bar{n}\langle M\rangle \cdot P\}
\end{aligned}
$$

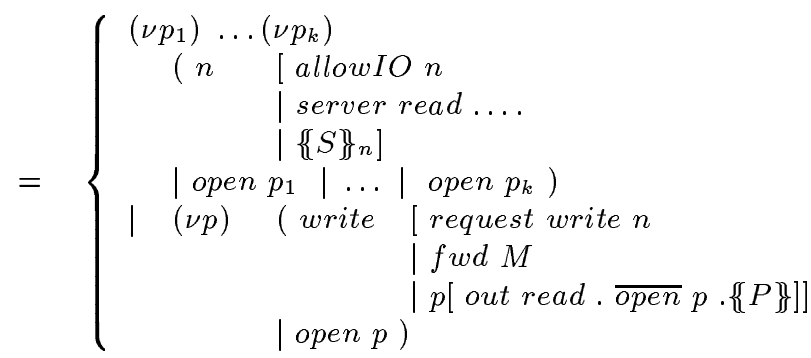

$$
\begin{aligned}
& \stackrel{p r}{\hookrightarrow}\left\{\begin{aligned}
&\left(\nu p_{1}\right) \ldots\left(\nu p_{k}\right)(\nu p) \\
& n {[\text { allowIO } n} \\
& \mid \text { server read } \ldots . . \\
& \mid\{S\}_{n} \\
& \mid \text { write } {[\overline{\text { in }} \text { write open enter }} \\
& \mid \text { fwd } M \\
&\mid p[\text { out read } . \overline{\text { open }} p \cdot\{[P\}]]] \\
&\left.\mid \text { open } p_{1}|\ldots| \text { open } p_{k} \mid \text { open } p\right)
\end{aligned}\right. \\
& =\{[n: S \mid\langle M\rangle \cdot P]\}
\end{aligned}
$$

Then, we can easily derive $\{\sigma,[n: S] \mid \bar{n}\langle M\rangle . P\} \stackrel{p r}{\hookrightarrow} \stackrel{a u x *}{\hookrightarrow}\{\{\sigma,[n: S \mid\langle M\rangle . P]\}$ for any $\sigma$. 
( $\pi_{\text {esc }}$ Red Input) Suppose that $\sigma:[n: S] \mid n(x) . P \longmapsto[n: S \mid(x) . P]$. If we name $p_{1}, \ldots, p_{k}$ the fresh names in $S$, and if we choose them as well as $p$ to avoid interferences, we have:

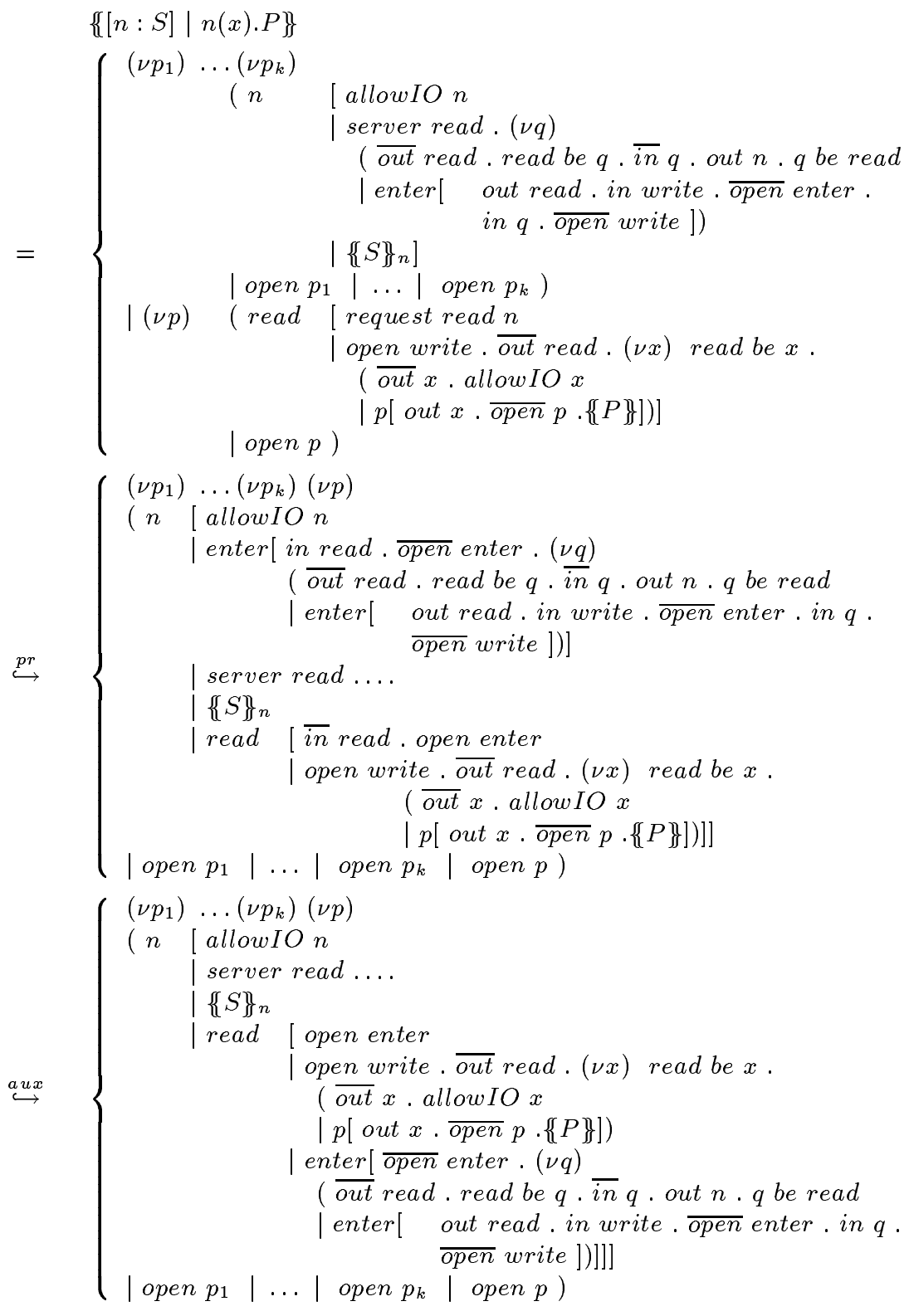




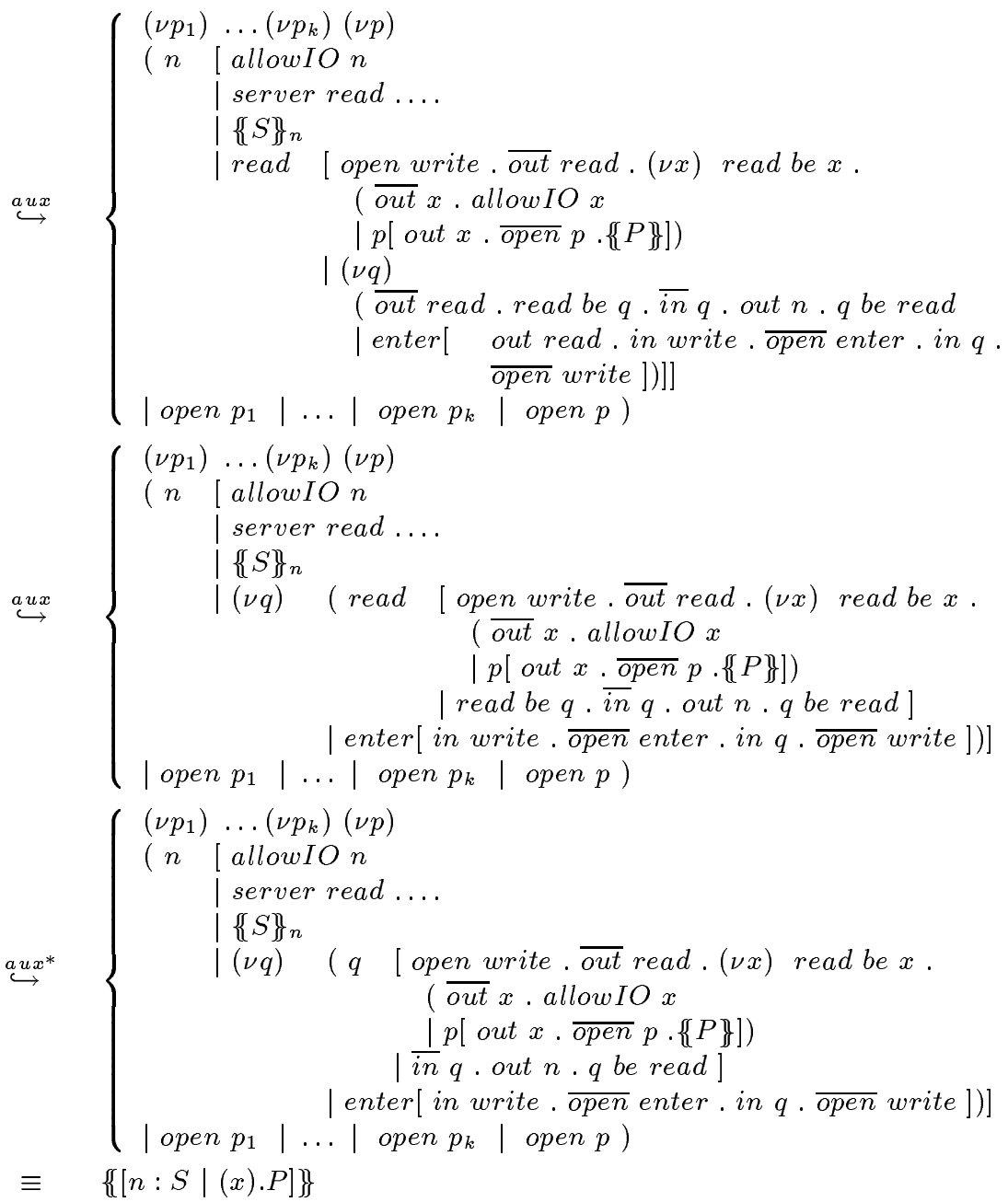

Then, we can easily derive $\{\sigma,[n: S] \mid n(x) . P\} \stackrel{p r}{\hookrightarrow} \stackrel{a u x *}{\hookrightarrow}\{\{\sigma[n: S \mid(x) . P]\}$ for any $\sigma$.

( $\pi_{e s c}$ Red Comm) Suppose that $\sigma:[n: S|\langle M\rangle . P|(x) . Q] \longmapsto[n: S]|P|(\nu x: M) Q$ with $x \neq M$. If we name $p_{1}, \ldots, p_{k}$ the fresh names in $S$, and if we choose them as well as $r_{1}$ and $r_{2}$ to avoid interferences, we have:

$$
\{[n: S|\langle M\rangle \cdot P|(x) \cdot Q]\}
$$




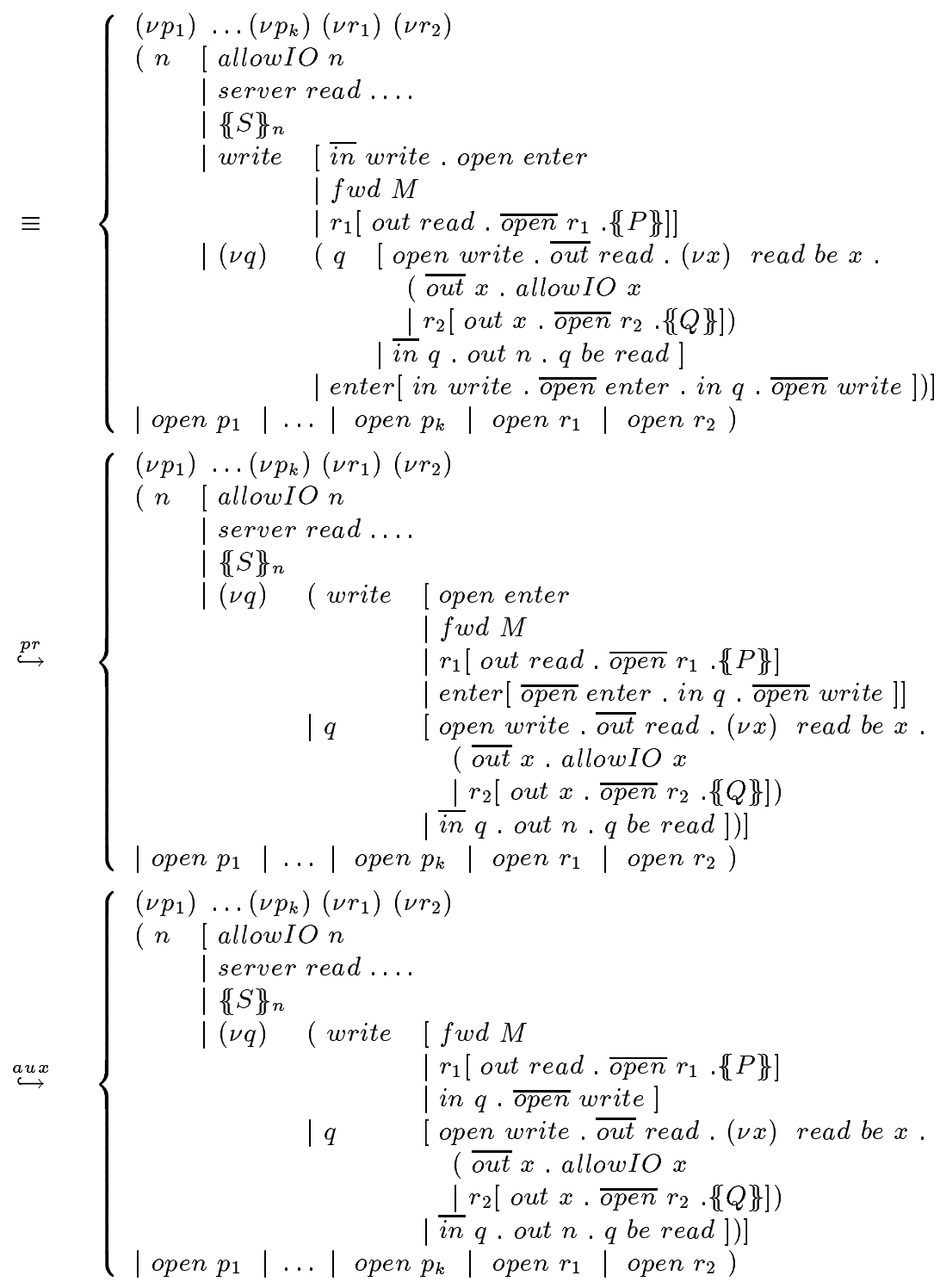




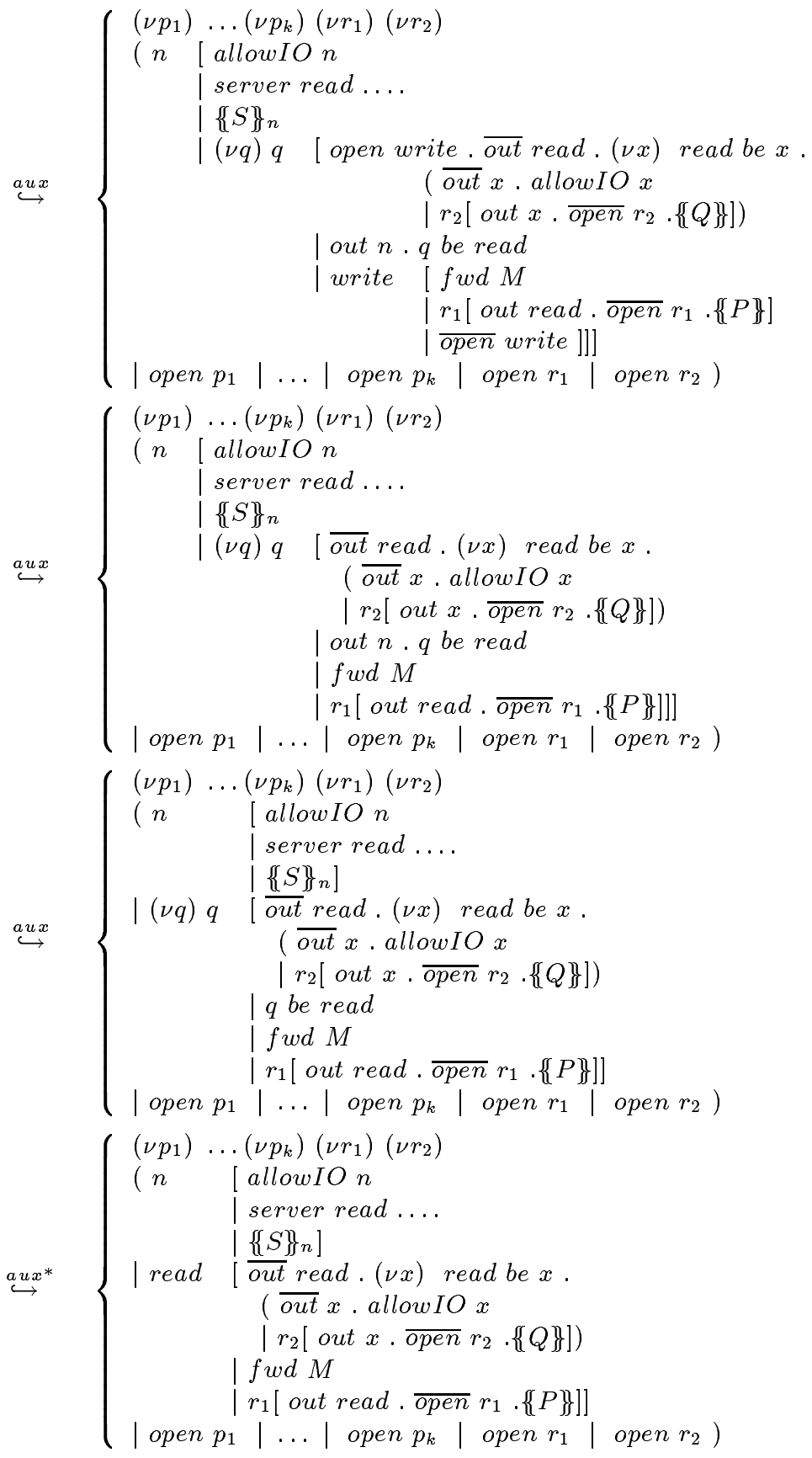




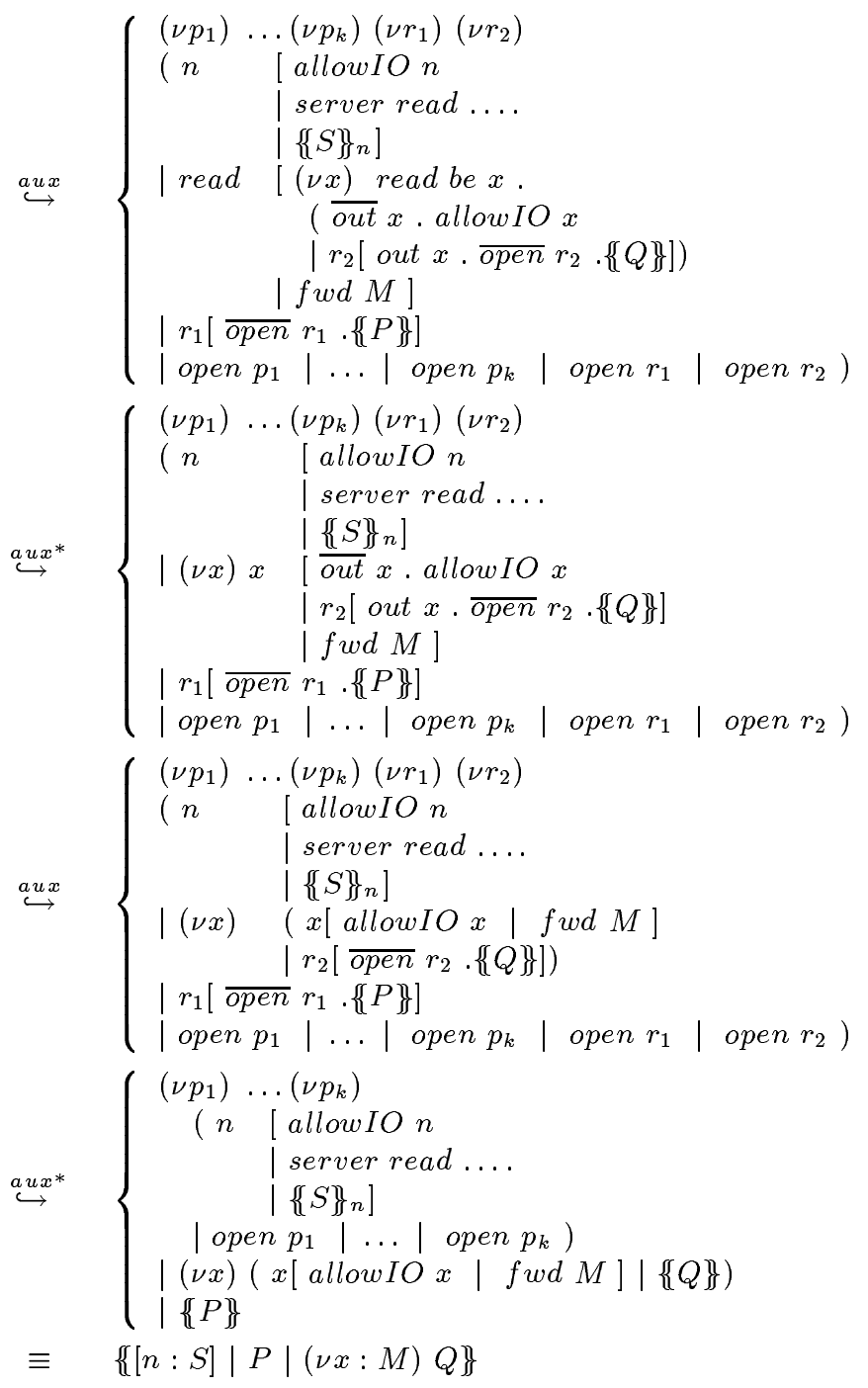

Then, we can easily derive $\{\sigma,[n: S|\langle M\rangle \cdot P|(x) \cdot Q]\} \stackrel{p r}{\hookrightarrow} \stackrel{a u x *}{\hookrightarrow}\{[\sigma,[n: S]|P|(\nu x: M) Q\}$ for any $\sigma$.

The other cases are similar or trivial.

$\mathrm{RR} \mathrm{n}^{\circ} 4350$ 


\section{References}

[Cardelli, 1999a] Cardelli, L. (1999a). Abstractions for Mobile Computation. In Vitek, J. and (Eds.), C. J., editors, Secure Internet Programming: Security Issues for Distributed and Mobile Objects, volume 1603 of Lecture Notes in Computer Science, pages 51-94. Springer Verlag.

[Cardelli, 1999b] Cardelli, L. (1999b). Wide Area Computation. In Proceedings of ICALP'99, volume 1644 of Lecture Notes in Computer Science, pages 10-24. Springer Verlag.

[Cardelli and Gordon, 1997] Cardelli, L. and Gordon, A. D. (1997). A Calculus of mobile Ambients. Slides.

[Cardelli and Gordon, 1998] Cardelli, L. and Gordon, A. D. (1998). Mobile Ambients. In Proceedings FoSSaCS'98, volume 1378 of Lecture Notes in Computer Science, pages 140155. Springer Verlag.

[Ferrari et al., 1996] Ferrari, G., Montanari, U., and Quaglia, P. (1996). A $\pi$-Calculus with Explicit Substitutions. Theoretical Computer Science, 168 (1):53-103.

[Hirschkoff, 1999] Hirschkoff, D. (1999). Handling Substitutions Explicitly in the $\pi$-Calculus. In Proceedings of the Floc Workshop WESTAPP 99.

[Honda and Yoshida, 1995] Honda, K. and Yoshida, N. (1995). On Reduction-Based Process Semantics. Theoretical Computer Science, 152:437-486.

[Levi and Sangiorgi, 2000] Levi, F. and Sangiorgi, D. (2000). Controlling Interference in Ambients. In Proceedings of POPL'00. ACM Press.

[Merro, 1999] Merro, M. (1999). On Equators in Asynchronous Name-Passing Calculi without Matching. In Proceedings of EXPRESS'g9, volume 27 of Electronic Notes in Theoretical Science. Elsevier.

[Merro and Sangiorgi, 1998] Merro, M. and Sangiorgi, D. (1998). On Asynchrony in NamePassing Calculi. In Proceedings of ICALP'98, volume 1443 of Lecture Notes in Computer Science, pages 856-867. Springer Verlag.

[Milner, 1991] Milner, R. (1991). The Polyadic $\pi$-Calculus: a Tutorial. Technical Report ECS-LFCS-91-180, University of Edinburgh.

[Palamidessi, 1997] Palamidessi, C. (1997). Comparing the Expressive Power of the Synchronous and the Asynchronous $\pi$-Calculus. In Proceedings of POPL'97, pages 256-265. ACM. 


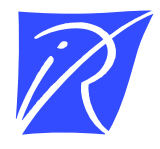

Unité de recherche INRIA Sophia Antipolis 2004, route des Lucioles - BP 93 - 06902 Sophia Antipolis Cedex (France)

Unité de recherche INRIA Lorraine : LORIA, Technopôle de Nancy-Brabois - Campus scientifique 615, rue du Jardin Botanique - BP 101 - 54602 Villers-lès-Nancy Cedex (France)

Unité de recherche INRIA Rennes : IRISA, Campus universitaire de Beaulieu - 35042 Rennes Cedex (France)

Unité de recherche INRIA Rhône-Alpes : 655, avenue de l'Europe - 38330 Montbonnot-St-Martin (France)

Unité de recherche INRIA Rocquencourt : Domaine de Voluceau - Rocquencourt - BP 105 - 78153 Le Chesnay Cedex (France)

Éditeur

INRIA - Domaine de Voluceau - Rocquencourt, BP 105 - 78153 Le Chesnay Cedex (France)

http://www.inria.fr

ISSN 0249-6399 